\title{
Replication of the Enzymatic Temperature Dependency of the Primary Hydride Kinetic Isotope Effects in Solution: Caused by the Protein Controlled Rigidity of the Donor-Acceptor Centers?
}

\author{
Yun Lu, ${ }^{*}$ Samantha Wilhelm, ${ }^{\dagger}$ Mingxuan Bai, ${ }^{\dagger}$ Peter Maness, Li Ma \\ Department of Chemistry, Southern Illinois University Edwardsville, Edwardsville IL 62026 \\ († The two authors contributed equally to this work)
}

Table S1. The activation parameters for the hydride transfer reactions in acetonitrile

S2

Figure S1. Exponential fit to the temperature dependences of $1^{\circ} \mathrm{KIEs}$ of hydride transfer reactions from various NADH models to $\mathrm{MA}^{+}$in acetonitrile S2

Table S2. The rate constants of the hydride versus deuteride transfer reactions from $\mathrm{BNAH}$ to $\mathrm{MA}^{+}$and the corresponding $1^{\circ}$

KIEs at different temperatures in acetonitrile S2

Table S3. The rate constants of the hydride versus deuteride transfer reactions from $\mathrm{HEH}$ to $\mathrm{MA}^{+}$and the corresponding $1^{\circ} \mathrm{KIEs}$ at different temperatures in acetonitrile

Table S4. The rate constants of the hydride versus deuteride transfer reactions from DMPBIH to $\mathrm{MA}^{+}$and the corresponding $1^{\circ}$

KIEs at different temperatures in acetonitrile S3

Table S5. The rate constants of the hydride versus deuteride transfer reactions from TMBIH to $\mathrm{MA}^{+}$and the corresponding $1^{\circ}$

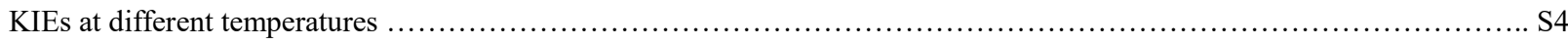

Figure S2. The CT absorption bands of the hydride transfer systems and decay with time in acetonitrile at $25^{\circ} \mathrm{C} \ldots \ldots \ldots \ldots . \mathrm{S} 4$

Table S6. The productive reactant complexes for the hydride transfer reaction from BNAH to $\mathrm{MA}^{+}$and relative properties ...S5

Table S7. The productive reactant complexes for the hydride transfer reaction from $\mathrm{HEH}$ to $\mathrm{MA}^{+}$and relative properties ..... S5

Table S8. The productive reactant complexes for the hydride transfer reaction from TMBIH to $\mathrm{MA}^{+}$and relative properties ..S7

Table S9. The productive reactant complexes for the hydride transfer reaction from DMPBIH to $\mathrm{MA}^{+}$and relative properties.S7

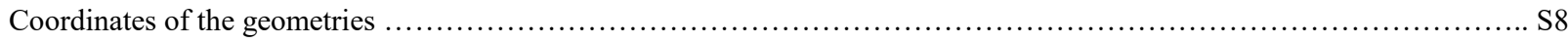

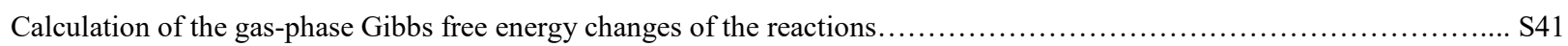

Synthesis of 1,2,3-trimethylbenzimidazoline (TMBIH) and 1,2,3-trimethyl-2-deutero-benzimidazoline (TMBIH-2-d)........ S47

${ }^{1} \mathrm{H}$ NMRs for TMBIH, TMBIH-2-d, HEH, HEH-4,4-d,d, DMPBIH, DMPBIH-2-d ...................................... S48

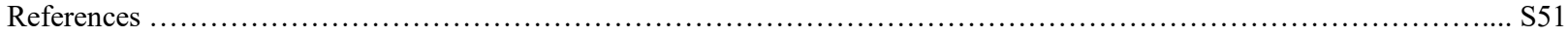


Table S1. The enthalpies and entropies of activation for the hydride transfer reactions in acetonitrile

\begin{tabular}{|c|c|c|c|c|c|c|c|c|}
\hline Reaction System & $\begin{array}{c}\mathrm{BNAH} / \\
\mathrm{MA}^{+} \mathrm{BF}_{4}^{-\mathrm{a}, \mathrm{b}}\end{array}$ & $\begin{array}{l}\mathrm{BNAH} / \\
\mathrm{MA}^{+} \mathrm{I}^{-\mathrm{a}, \mathrm{c}}\end{array}$ & $\begin{array}{c}\mathrm{CH}_{3} \mathrm{BNAH} / \\
\mathrm{MA}^{+} \mathrm{I}^{-\mathrm{d}}\end{array}$ & $\begin{array}{c}\mathrm{HEH} / \\
\mathrm{MA}^{+} \mathrm{BF}_{4}^{-\mathrm{a}}\end{array}$ & $\begin{array}{c}\mathrm{HEH} / \\
\mathrm{MA}^{+} \mathrm{BF}_{4}^{-\mathrm{e}}\end{array}$ & $\begin{array}{l}\mathrm{DMPBIH} / \\
\mathrm{MA}^{+} \mathrm{BF}_{4}^{-\mathrm{a}}\end{array}$ & $\begin{array}{l}\mathrm{DMPBIH} / \\
\mathrm{MA}^{+} \mathrm{BF}_{4}^{-\mathrm{e}}\end{array}$ & $\begin{array}{c}\mathrm{TMBIH} / \\
\mathrm{MA}^{+} \mathrm{BF}_{4}^{-\mathrm{e}}\end{array}$ \\
\hline Column & 1 & 2 & 3 & 4 & 5 & 6 & 7 & 8 \\
\hline$\Delta H^{\ddagger}{ }_{\mathrm{H}}(\mathrm{kcal} / \mathrm{mol})$ & $4.84(0.17)$ & $4.20(0.11)$ & $7.66^{f}$ & $4.69(0.04)$ & $5.71(0.03)$ & $5.79(0.11)$ & $7.20(0.07)$ & $7.01(0.07)$ \\
\hline$\Delta S_{\mathrm{H}}^{\ddagger}(\mathrm{cal} / \mathrm{mol} \mathrm{K})$ & $\begin{array}{r}-34.03 \\
(0.59)\end{array}$ & $\begin{array}{c}-36.44 \\
(0.38)\end{array}$ & $-28.69^{f}$ & $\begin{array}{r}-33.08 \\
(0.14)\end{array}$ & $\begin{array}{l}-29.53 \\
(0.10)\end{array}$ & $\begin{array}{l}-28.79 \\
(0.37)\end{array}$ & $\begin{array}{l}-23.93 \\
(0.24)\end{array}$ & $\begin{array}{c}-21.00 \\
(0.23)\end{array}$ \\
\hline $\begin{array}{l}{ }^{\text {a }} \text { Using the a Hi-T } \\
\text { footnote e was not } \\
\text { reported for entha } \\
\text { deviations were re }\end{array}$ & $\begin{array}{l}\text { Ch Scientific } \\
\text { y and entropy } \\
\text { orted. }\end{array}$ & $\begin{array}{l}\text { A-20 fast kin } \\
\text { published wo } \\
\text { f activation; }\end{array}$ & $\begin{array}{l}\text { ic determinati } \\
1 ;{ }^{c} \text { from our } p \\
\text { sing a SF-61D }\end{array}$ & $\begin{array}{l}\text { kit connecte } \\
\text { vious unpubl } \\
2 \text { Hi-Tech K }\end{array}$ & $\begin{array}{l}\text { a spectroph } \\
\text { ed results; }{ }^{d} \\
\text { Asyst doubl }\end{array}$ & $\begin{array}{l}\text { neter (at time } \\
\text { reference }{ }^{2} \text {, } \\
\text { ixing stoppec }\end{array}$ & $\begin{array}{l}\text { en the instrun } \\
\text { dard deviatior } \\
\text { w instrument }\end{array}$ & $\begin{array}{l}\text { nt in } \\
\text { nos not } \\
\text { no standard }\end{array}$ \\
\hline
\end{tabular}

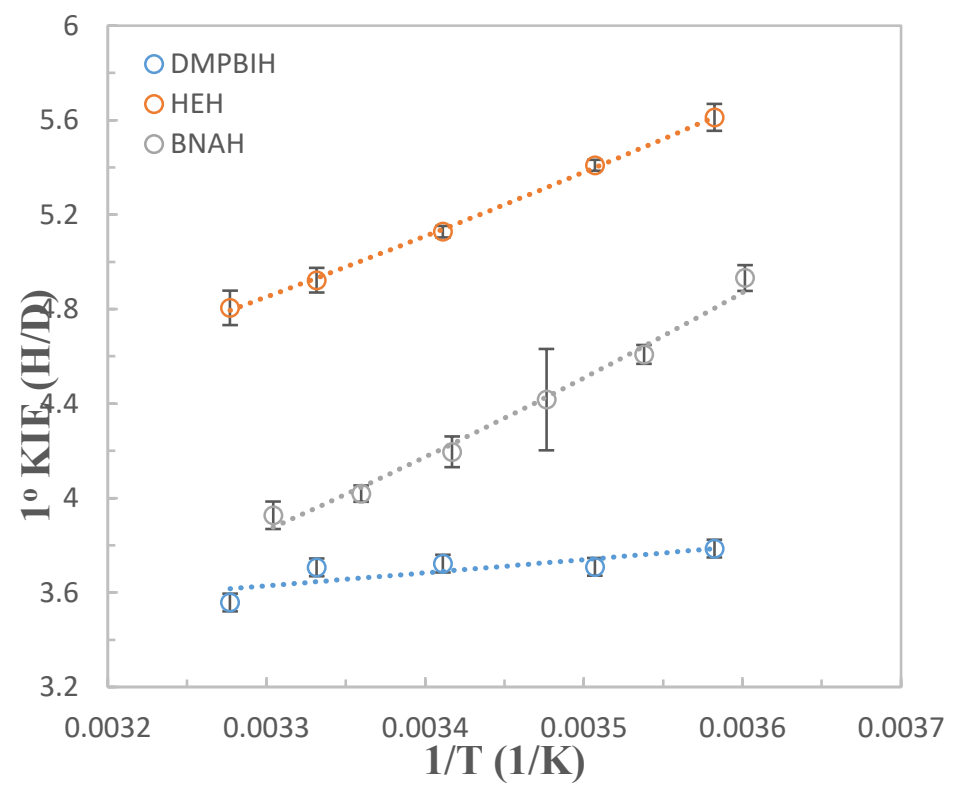

Figure S1. Arrhenius fit to the temperature dependences of the $1^{\circ} \mathrm{KIEs}$ of hydride transfer reactions from various NADH models to $\mathrm{MA}^{+}$in acetonitrile (data corresponding to columns 1, 4, 6 of Table 1 in the main paper). For the TMBIH reaction, there are data measured in this temperature range.

Table S2. The rate constants of the hydride versus deuteride transfer reactions from $\mathrm{BNAH}$ to $\mathrm{MA}^{+}$and the corresponding $1^{\circ}$ KIEs at different temperatures in acetonitrile

\begin{tabular}{lccc}
\hline Temp $\left({ }^{\circ} \mathbf{C}\right)$ & $\boldsymbol{k}_{\mathbf{H}}\left(\mathbf{M}^{-1} \mathbf{s}^{-1}\right)$ & $\boldsymbol{k}_{\mathbf{D}}\left(\mathbf{M}^{-1} \mathbf{s}^{-1}\right)$ & $\mathbf{1}^{\circ} \mathbf{K I E}$ \\
\hline With $\mathrm{MA}^{+} \mathrm{BF}_{4}^{-}$, determined from a Hi-Tech Scientific SFA-20 fast kinetic determination kit connected to a spectrophotometer & ${ }^{\mathrm{a}}$ \\
29.5 & $8.30 \times 10(1.04)$ & $2.11 \times 10(0.10)$ & $3.93(0.06)$ \\
24.5 & $6.97 \times 10(0.33)$ & $1.73 \times 10(0.12)$ & $4.02(0.03)$ \\
19.5 & $5.92 \times 10(0.44)$ & $1.41 \times 10(0.17)$ & $4.20(0.07)$ \\
14.5 & $5.20 \times 10(0.40)$ & $1.18 \times 10(0.49)$ & $4.42(0.21)$ \\
9.5 & $4.27 \times 10(0.31)$ & $9.27(0.04)$ & $4.61(0.04)$ \\
4.5 & $3.68 \times 10(0.32)$ & $7.48(0.04)$ & $4.93(0.05)$
\end{tabular}


With $\mathrm{MA}^{+} \mathrm{I}^{-}$, determined from a Hi-Tech Scientific SFA-20 fast kinetic determination kit connected to a spectrophotometer ${ }^{\mathrm{a}}$

$\begin{array}{llll}29.5 & 7.07 \times 10(2.26) & 1.89 \times 10(0.12) & 3.74(0.12) \\ 24.5 & 6.14 \times 10(0.99) & 1.52 \times 10(0.07) & 4.04(0.07) \\ 19.5 & 5.32 \times 10(1.09) & 1.22 \times 10(0.09) & 4.36(0.11) \\ 14.5 & 4.64 \times 10(1.18) & 1.02 \times 10(0.05) & 4.54(0.13) \\ 9.5 & 4.05 \times 10(0.65) & 8.23(0.04) & 4.93(0.09) \\ 4.5 & 3.44 \times 10(0.09) & 7.07(0.09) & 4.87(0.07)\end{array}$

${ }^{a}$ Repeated on two different days with 4-6 repetitions each day. Numbers in parentheses are the pooled standard deviations S(pooled).

Table S3. The rate constants of the hydride versus deuteride transfer reactions from $\mathrm{HEH}$ to $\mathrm{MA}^{+} \mathrm{BF}_{4}^{-}$and the corresponding $1^{\circ}$ KIEs at different temperatures in acetonitrile

\begin{tabular}{lccc}
\hline Temp $\left({ }^{\circ} \mathbf{C}\right)$ & $\boldsymbol{k}_{\mathbf{H}}\left(\mathbf{M}^{-1} \mathbf{s}^{-1}\right)$ & $\boldsymbol{k}_{\mathbf{D}}\left(\mathbf{M}^{-1} \mathbf{s}^{-1}\right)$ & $\mathbf{1}^{\circ} \mathbf{K I E}$ \\
\hline Determined from a SF-61DX2 Hi-Tech KinetAsyst double-mixing stopped-flow spectrophotometer & ${ }^{\mathrm{a}}$ & \\
45.0 & $3.07 \times 10^{2}(1.26)$ & $6.95 \times 10(0.42)$ & $4.42(0.03)$ \\
35.0 & $2.22 \times 10^{2}(1.02)$ & $4.76 \times 10(0.31)$ & $4.67(0.04)$ \\
25.0 & $1.56 \times 10^{2}(1.05)$ & $3.18 \times 10(0.12)$ & $4.92(0.04)$ \\
15.0 & $1.09 \times 10^{2}(0.51)$ & $2.06 \times 10(0.10)$ & $5.31(0.04)$ \\
5.2 & $7.43 \times 10(0.66)$ & $1.37 \times 10(0.25)$ & $5.41(0.12)$
\end{tabular}

Determined from a Hi-Tech Scientific SFA-20 fast kinetic determination kit connected to a spectrophotometer ${ }^{\text {a }}$

$\begin{array}{lllr}32.0 & 1.80 \times 10^{2}(1.16) & 3.74 \times 10(0.48) & 4.81(0.07) \\ 27.0 & 1.57 \times 10^{2}(1.05) & 3.18 \times 10(0.24) & 4.92(0.05) \\ 20.0 & 1.27 \times 10^{2}(0.38) & 2.48 \times 10(0.08) & 5.13(0.02) \\ 12.0 & 9.79 \times 10(0.30) & 1.81 \times 10(0.05) & 5.41(0.02) \\ 6.0 & 8.04 \times 10(0.56) & 1.43 \times 10(0.10) & 5.61(0.06)\end{array}$

${ }^{a}$ Repeated on two different days with 6 repetitions each day. Numbers in parentheses are the pooled standard deviations S(pooled).

Table S4. The rate constants of the hydride versus deuteride transfer reactions from $\mathrm{DMPBIH}$ to $\mathrm{MA}^{+} \mathrm{BF}_{4}^{-}$and the corresponding $1^{\circ} \mathrm{KIEs}$ at different temperatures in acetonitrile

\begin{tabular}{lccc}
\hline Temp $\left({ }^{\circ} \mathbf{C}\right)$ & $\boldsymbol{k}_{\mathbf{H}}\left(\mathbf{M}^{-1} \mathbf{s}^{-1}\right)$ & $\boldsymbol{k}_{\mathbf{D}}\left(\mathbf{M}^{-1} \mathbf{s}^{-\mathbf{1}}\right)$ & $\mathbf{1}^{\circ} \mathbf{K I E}$ \\
\hline Determined from a SF-61DX2 Hi-Tech KinetAsyst double-mixing stopped-flow spectrophotometer ${ }^{\text {a }}$ & \\
45.0 & $4.92 \times 10^{2}(1.71)$ & $1.46 \times 10^{2}(1.00)$ & $3.37(0.03)$ \\
35.0 & $3.26 \times 10^{2}(1.48)$ & $9.41 \times 10(0.59)$ & $3.47(0.03)$ \\
25.0 & $2.12 \times 10^{2}(1.15)$ & $5.92 \times 10(0.43)$ & $3.57(0.03)$ \\
15.0 & $1.34 \times 10^{2}(0.38)$ & $3.64 \times 10(0.23)$ & $3.69(0.03)$ \\
5.2 & $8.47 \times 10(0.52)$ & $2.29 \times 10(0.11)$ & $3.70(0.03)$ \\
& & $3.56(0.08)$ \\
Determined from a Hi-Tech Scientific SFA-20 fast kinetic determination kit connected to a spectrophotometer & \\
32.0 & $2.51 \times 10^{2}(3.76)$ & $7.06 \times 10(1.10)$ & $3.71(0.07)$ \\
27.0 & $2.15 \times 10^{2}(3.34)$ & $5.81 \times 10(0.66)$ & $3.72(0.03)$ \\
20.0 & $1.65 \times 10^{2}(0.88)$ & $4.44 \times 10(0.27)$ & $3.71(0.04)$ \\
12.0 & $1.21 \times 10^{2}(0.67)$ & $3.26 \times 10(0.28)$ & $3.79(0.05)$ \\
6.0 & $9.57 \times 10(0.98)$ & $2.53 \times 10(0.14)$ & \\
${ }^{a}$ Repeated on two different days with 6 repetitions each day. Numbers in parentheses are the pooled standard deviations S(pooled).
\end{tabular}


Table S5. The rate constants of the hydride versus deuteride transfer reactions from $\mathrm{TMBIH}$ to $\mathrm{MA}^{+} \mathrm{BF}_{4}^{-}$and the corresponding $1^{\circ}$ KIEs at different temperatures ${ }^{\text {a }}$

\begin{tabular}{llll}
\hline Temp $\left({ }^{\circ} \mathbf{C}\right)$ & $\boldsymbol{k}_{\mathbf{H}}\left(\mathbf{M}^{-1} \mathbf{s}^{-1}\right)$ & $\boldsymbol{k}_{\mathbf{D}}\left(\mathbf{M}^{-1} \mathbf{s}^{-1}\right)$ & $\mathbf{1}^{\circ} \mathbf{K I E}$ \\
\hline Determined from a SF-61DX2 Hi-Tech KinetAsyst double-mixing stopped-flow spectrophotometer & \\
45.0 & $2.81 \times 10^{3}(9.07)$ & $1.12 \times 10^{3}(7.91)$ & $2.52(0.02)$ \\
35.0 & $1.92 \times 10^{3}(29.7)$ & $7.50 \times 10^{2}(4.87)$ & $2.58(0.04)$ \\
25.0 & $1.28 \times 10^{3}(4.91)$ & $4.74 \times 10^{2}(2.71)$ & $2.71(0.02)$ \\
15.0 & $8.13 \times 10^{2}(4.30)$ & $2.94 \times 10^{2}(1.87)$ & $2.77(0.02)$ \\
5.0 & $5.09 \times 10^{2}(2.62)$ & $1.80 \times 10^{2}(0.89)$ & $2.84(0.02)$
\end{tabular}

${ }^{\text {a }}$ Repeated on three different days with 6 repetitions each day. Numbers in parentheses are the pooled standard deviations S(pooled).

Table S6. Kinetic data for determination of the $\alpha-2^{\circ}$ KIEs on $\mathrm{MA}^{+} \mathrm{BF}_{4}{ }^{-}$and standard deviations (SD's) for its reaction with TMBIH in acetonitrile (corresponding with Table 2 in the paper)

\begin{tabular}{llll}
\hline \# of Measurement & $\begin{array}{l}k(9-\mathrm{H}) \\
\left(10^{2} \mathrm{M}^{-1} \mathrm{~s}^{-1}\right)^{\mathrm{a}}\end{array}$ & $\begin{array}{l}k(9-\mathrm{D}) \\
\left(10^{2} \mathrm{M}^{-1} \mathrm{~s}^{-1}\right)^{\mathrm{a}}\end{array}$ & $2^{\mathrm{o}} \mathrm{KIE}^{\mathrm{b}}$ \\
\hline 1 & $4.784(0.015)$ & $4.863(0.027)$ & $0.984(0.006)$ \\
2 & $4.766(0.026)$ & $4.741(0.019)$ & $1.005(0.007)$ \\
3 & $4.779(0.023)$ & $4.776(0.029)$ & $1.001(0.008)$ \\
& & & $\begin{array}{l}\text { Average } \\
\end{array}$ \\
& & & $0.997(0.007)^{\mathrm{c}}$
\end{tabular}

a at $35^{\circ} \mathrm{C}$, each rate constant came from 6 measurements; ${ }^{\mathrm{b}} \mathrm{SD}=\mathrm{KIE} \cdot\left(\left(\mathrm{SD}\left(\mathrm{CH}_{3}\right) / k\left(\mathrm{CH}_{3}\right)\right)^{2}+\left(\mathrm{SD}\left(\mathrm{CD}_{3}\right) / k\left(\mathrm{CD}_{3}\right)^{2}\right)^{1 / 2}\right.$, otherwise indicated; ${ }^{\mathrm{c}}$ numbers in parentheses are the pooled standard deviations $\mathrm{S}$ (pooled).
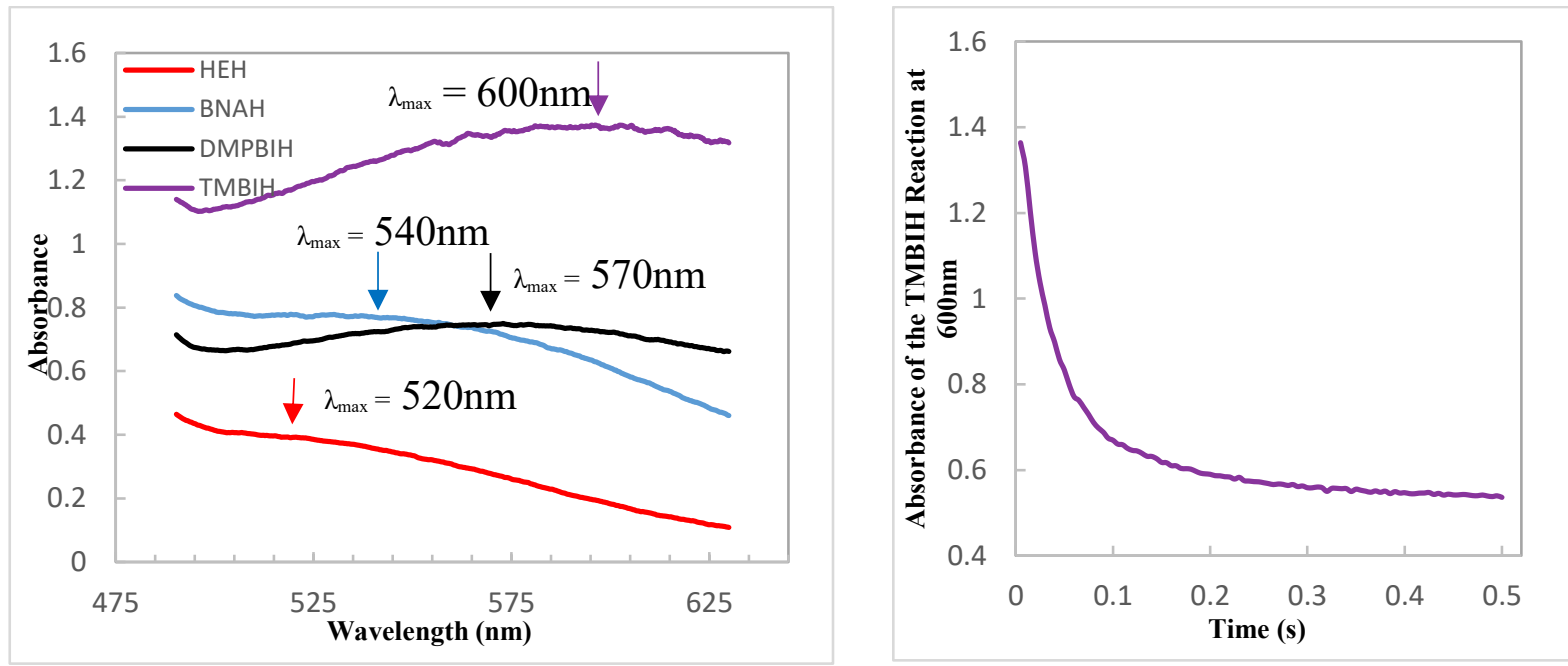

Figure S2. Left: The CT absorption bands of the hydride transfer systems in acetonitrile at $25{ }^{\circ} \mathrm{C}$, measured upon mixing of the reactant solutions. The concentration conditions are $\mathrm{HEH}(0.02 \mathrm{M}) / \mathrm{MA}^{+}(0.06 \mathrm{M}), \mathrm{BNAH}(0.12 \mathrm{M}) / \mathrm{MA}^{+}(0.12 \mathrm{M}), \mathrm{DMPBIH}(0.12$ $\mathrm{M}) / \mathrm{MA}^{+}(0.08 \mathrm{M}), \mathrm{TMBIH}(0.12 \mathrm{M}) / \mathrm{MA}^{+}(0.16 \mathrm{M})$. Right: The decay of the CT absorption at $600 \mathrm{~nm}$ with time for the reaction of $\mathrm{TMBIH}(0.12 \mathrm{M})$ and $\mathrm{MA}^{+}(0.12 \mathrm{M})$ at $25^{\circ} \mathrm{C}$. 
Table S7. The productive reactant complexes for the hydride transfer reaction from BNAH to $\mathrm{MA}^{+}$and relative properties (excluding their mirror images (enantiomers)) $\left(25^{\circ} \mathrm{C}\right)$

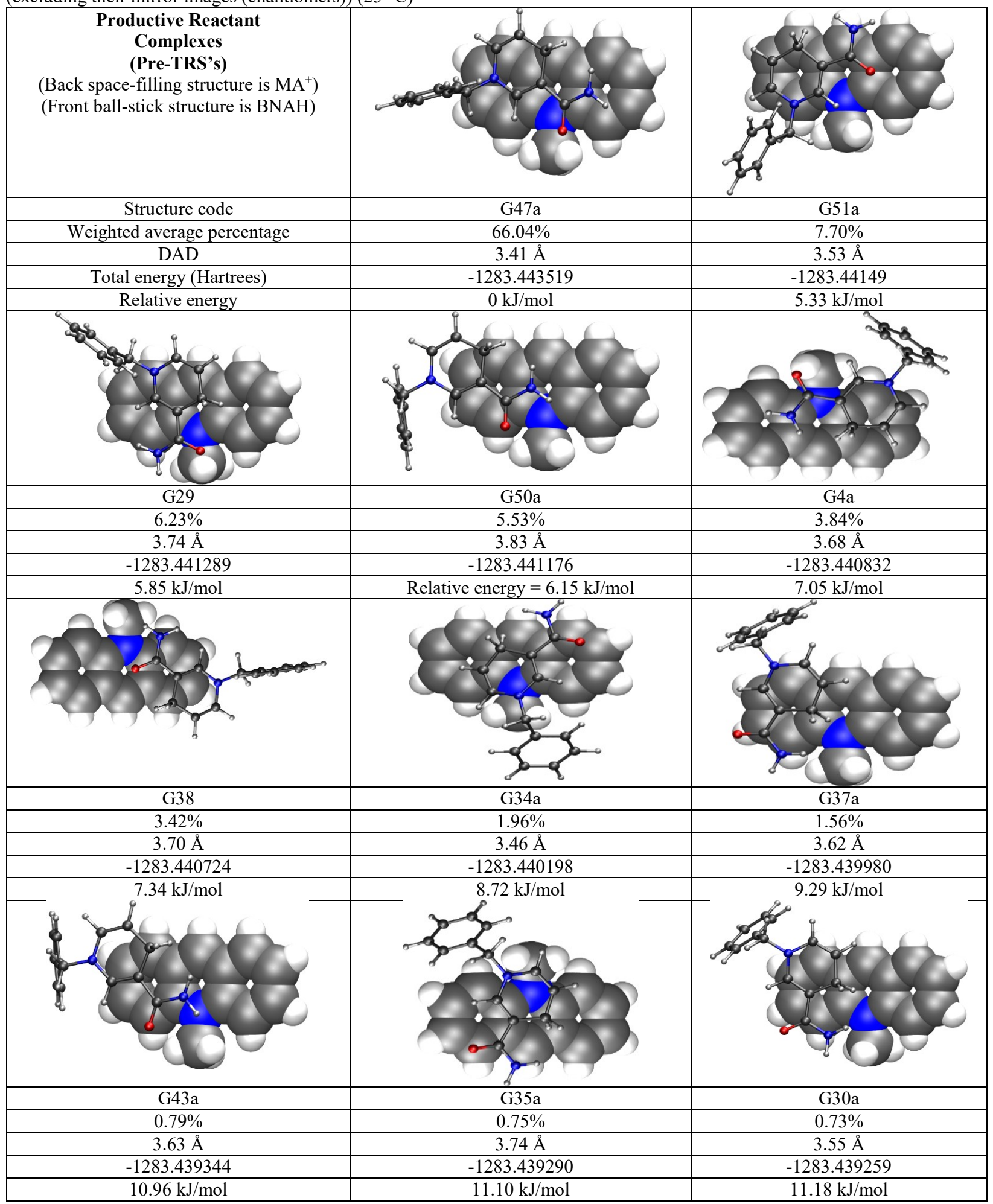




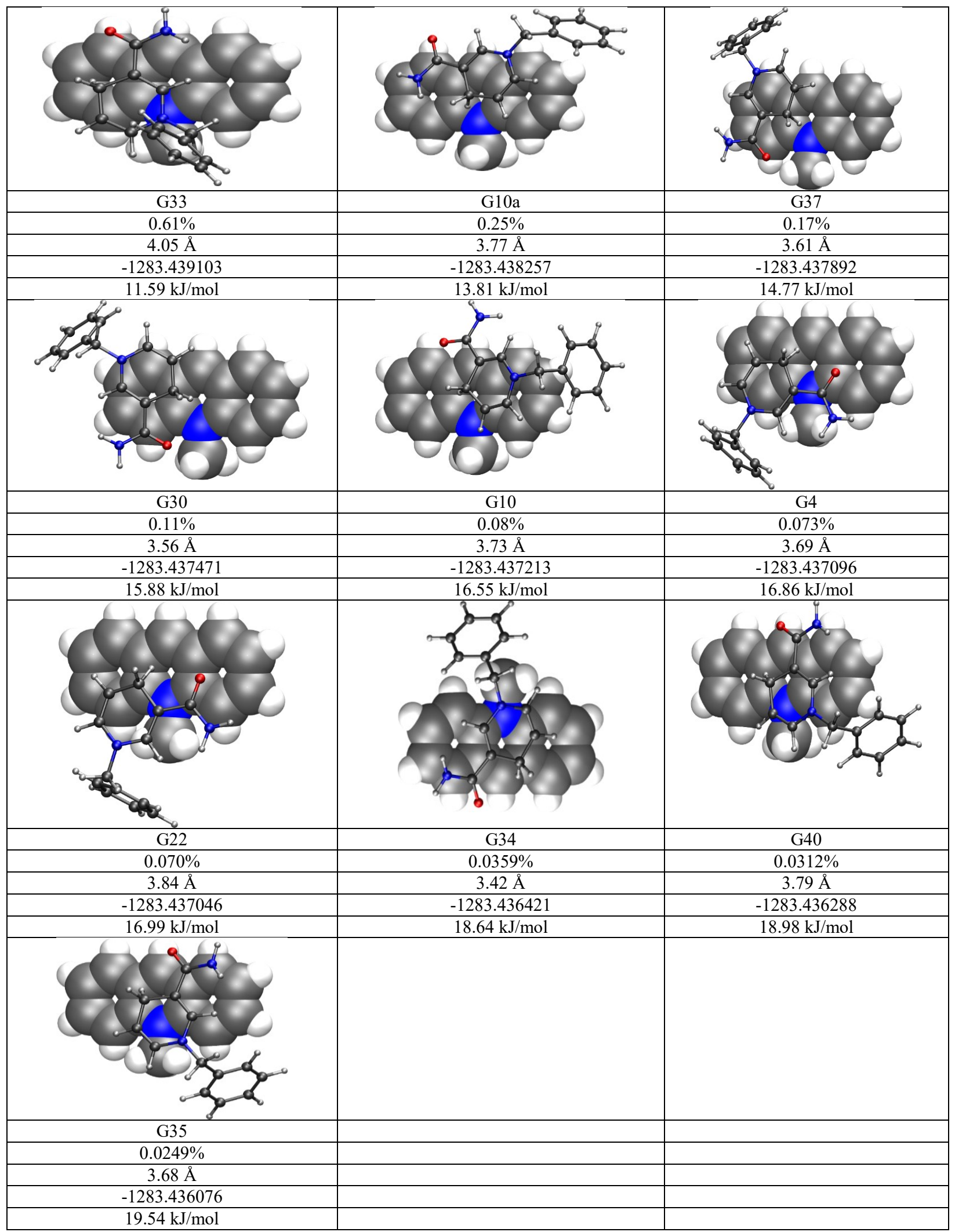


Table S8. The productive reactant complexes for the hydride transfer reaction from $\mathrm{HEH}$ to $\mathrm{MA}^{+}$and relative properties (excluding their mirror images (enantiomers)) $\left(25^{\circ} \mathrm{C}\right)$

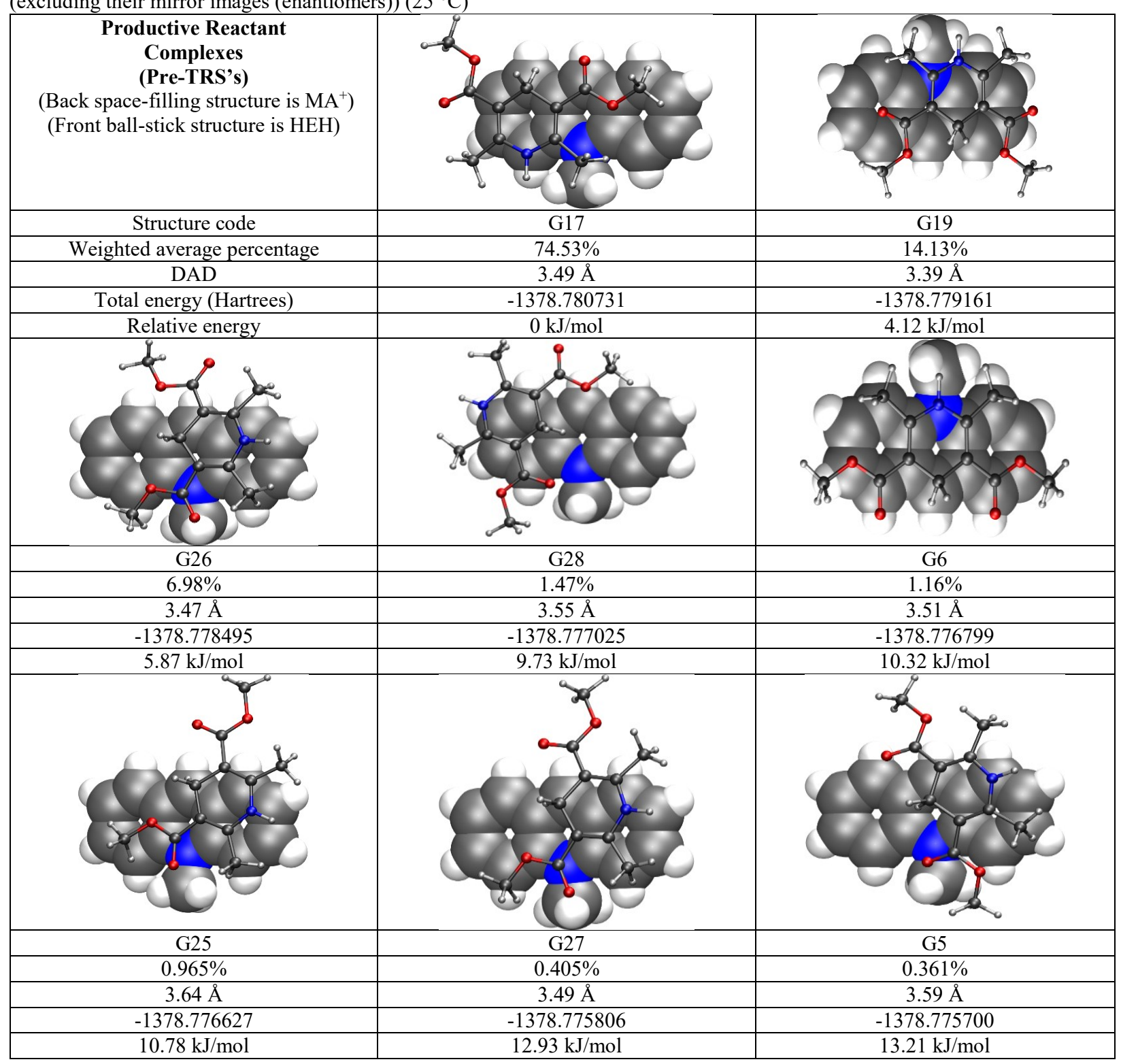

Table S9. The productive reactant complexes for the hydride transfer reaction from TMBIH to $\mathrm{MA}^{+}$(excluding their mirror images (enantiomers)) $\left(25^{\circ} \mathrm{C}\right)$

\begin{tabular}{|c|c|c|}
\hline $\begin{array}{c}\text { Productive Reactant } \\
\text { Complexes } \\
\text { (Pre-TRS's) } \\
\text { (Back space-filling structure is } \mathrm{MA}^{+} \text {) } \\
\text { (Front ball-stick structure is } \\
\text { TMPBIH) }\end{array}$ & & \\
\hline Structure code & G14 & G26 \\
\hline Weighted average percentage & $92.47 \%$ & $7.53 \%$ \\
\hline DAD & $3.55 \AA$ & $3.61 \AA$ \\
\hline Total energy (Hartrees) & -1093.893077 & -1093.890708 \\
\hline Relative energy & $0 \mathrm{~kJ} / \mathrm{mol}$ & $6.22 \mathrm{~kJ} / \mathrm{mol}$ \\
\hline
\end{tabular}


Table S10. The productive reactant complexes for the hydride transfer reaction from DMPBIH to $\mathrm{MA}^{+}$and relative properties (excluding their mirror images (enantiomers) $)\left(25^{\circ} \mathrm{C}\right)$

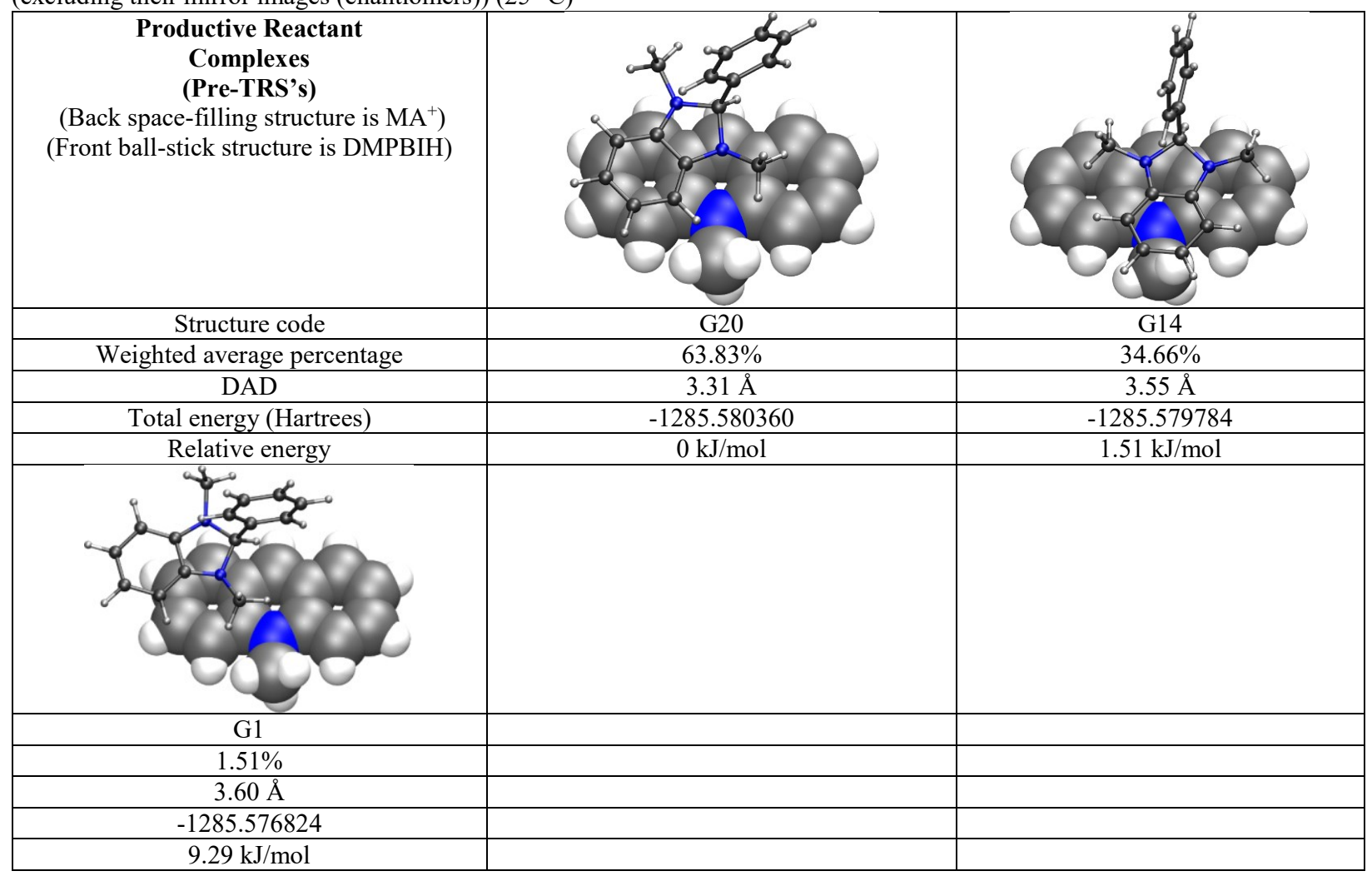

Coordinates of the geometries in Table S7 (corresponding total energies are listed in Table S7)

$\mathrm{MA}+$ \& BNAH: G10a

$\begin{array}{llll}\mathrm{H} & -1.438002295663 & 0.123896293885 & 1.792651124178 \\ \mathrm{C} & -0.674654098205 & 0.834805312159 & 2.140808571863 \\ \mathrm{C} & 0.578530242019 & 0.100422683824 & 2.533703434512 \\ \mathrm{H} & -1.115413777048 & 1.313042230004 & 3.025679305313 \\ \mathrm{C} & -0.367002680460 & 1.852286831153 & 1.065459919344 \\ \mathrm{C} & 1.776124994075 & 0.385461102478 & 2.026929959472 \\ \mathrm{C} & 0.889201827995 & 2.061556126081 & 0.618071227225 \\ \mathrm{C} & -1.442658336564 & 2.659150406111 & 0.462921731242 \\ \mathrm{~N} & 1.974588584705 & 1.377816236229 & 1.067267230992 \\ \mathrm{O} & -1.279620168768 & 3.401238182504 & -0.506774427570 \\ \mathrm{H} & -2.938087401897 & 1.782555143855 & -2.497486325700 \\ \mathrm{C} & -3.434193522152 & 1.108614285100 & -1.810869424664 \\ \mathrm{C} & -2.655812964537 & 0.077854124898 & -1.215245271450 \\ \mathrm{C} & -4.750047113678 & 1.242698656817 & -1.501044129811 \\ \mathrm{C} & -3.265009818820 & -0.844184109267 & -0.316576335304 \\ \mathrm{C} & -1.299383172796 & -0.037945188027 & -1.476315221189 \\ \mathrm{C} & -5.332983740827 & 0.361677180550 & -0.558648766897 \\ \mathrm{H} & -5.348965014588 & 2.025910637343 & -1.945611970701 \\ \mathrm{C} & -4.627185736809 & -0.655421034873 & 0.021198750974 \\ \mathrm{~N} & -2.527688409309 & -1.868948843418 & 0.202443059708 \\ \mathrm{C} & -0.539954115349 & -1.027672060271 & -0.865525647585 \\ \mathrm{H} & -6.370828429377 & 0.497542397991 & -0.281489388880 \\ \mathrm{H} & -5.113128744413 & -1.285464767537 & 0.748785856859 \\ \mathrm{C} & -1.177200832418 & -1.955634693938 & 0.004477218437 \\ \mathrm{C} & -3.203407387312 & -2.890152032721 & 1.014252703620 \\ \mathrm{C} & 0.859787774816 & -1.109607106065 & -1.089086785966 \\ \mathrm{C} & -0.377736421958 & -2.925671474523 & 0.654799607991\end{array}$




\begin{tabular}{|c|c|c|c|}
\hline $\mathrm{H}$ & -2.675483331704 & -3.830311000153 & 0.918208482861 \\
\hline $\mathrm{H}$ & -3.234807805290 & -2.579570229174 & 2.058555699682 \\
\hline $\mathrm{H}$ & -4.207535398384 & -3.038670818021 & 0.634912077130 \\
\hline $\mathrm{C}$ & 1.606035967004 & -2.057162835606 & -0.462151460055 \\
\hline $\mathrm{H}$ & 1.304589693490 & -0.389885800532 & -1.764291114143 \\
\hline $\mathrm{C}$ & 0.970170591477 & -2.960411164954 & 0.421624583698 \\
\hline $\mathrm{H}$ & -0.803208469831 & -3.620614473803 & 1.360549697122 \\
\hline $\mathrm{H}$ & 2.675993990242 & -2.113796208100 & -0.620713174308 \\
\hline $\mathrm{H}$ & 1.567357394022 & -3.699133471086 & 0.941363116649 \\
\hline $\mathrm{H}$ & -0.821410273408 & 0.661265537888 & -2.154020008790 \\
\hline $\mathrm{H}$ & 0.506784447854 & -0.688196523830 & 3.271733224439 \\
\hline $\mathrm{N}$ & -2.658253737949 & 2.534886394195 & 1.047255286393 \\
\hline $\mathrm{H}$ & -2.825034419595 & 1.942856981130 & 1.843582815887 \\
\hline $\mathrm{H}$ & -3.438332766323 & 3.034550354367 & 0.649453092539 \\
\hline $\mathrm{H}$ & 1.078426980030 & 2.810579680818 & -0.141036134592 \\
\hline $\mathrm{H}$ & 2.671637455399 & -0.143351792021 & 2.327541117356 \\
\hline $\mathrm{C}$ & 3.336400245348 & 1.731572891030 & 0.674569079821 \\
\hline $\mathrm{C}$ & 4.015595656620 & 0.652618464267 & -0.134863879739 \\
\hline $\mathrm{H}$ & 3.916950031391 & 1.935847121217 & 1.577046116782 \\
\hline $\mathrm{H}$ & 3.275762648004 & 2.651667417357 & 0.095393946761 \\
\hline $\mathrm{C}$ & 4.852070937708 & -0.276812761660 & 0.476526896811 \\
\hline $\mathrm{C}$ & 3.798131221991 & 0.565319832903 & -1.508212540111 \\
\hline $\mathrm{C}$ & 5.452524446146 & -1.285887097052 & -0.268411119156 \\
\hline $\mathrm{H}$ & 5.039647073820 & -0.204774482373 & 1.542180389060 \\
\hline $\mathrm{C}$ & 4.395203913839 & -0.4400418 & -2.255834306182 \\
\hline $\mathrm{H}$ & 3.156753183255 & 1.29 & -1.992347978229 \\
\hline $\mathrm{C}$ & 5.223454862126 & -1.369946329127 & -1.635439769226 \\
\hline $\mathrm{H}$ & 6.102222974019 & -2.002353439557 & 0.218769436346 \\
\hline $\mathrm{H}$ & 4.220119969940 & -0.496297591439 & -3.323190539341 \\
\hline $\mathrm{H}$ & 5.692155278096 & -2.153189388582 & -2.218053041474 \\
\hline & & & \\
\hline $\mathrm{H}$ & -1.526167504376 & 0.745760315320 & 1.342472982717 \\
\hline $\mathrm{C}$ & -0.664349835203 & 1.223466961647 & 1.819004496588 \\
\hline $\mathrm{C}$ & 0.208289854900 & 0.198943680 & 2.495335660420 \\
\hline $\mathrm{H}$ & -1.095994063289 & 1.912284656674 & 2.556470149820 \\
\hline $\mathrm{C}$ & 0.143030363723 & 1.980188737393 & 0.790081461071 \\
\hline $\mathrm{C}$ & 1.538192275868 & 0.231455913784 & 2.418235682298 \\
\hline $\mathrm{C}$ & 1.490786194530 & 1.956937079893 & 0.803172926675 \\
\hline $\mathrm{C}$ & -0.613184571339 & 2.788689663530 & -0.180606875162 \\
\hline $\mathrm{N}$ & 2.218156549682 & 1.186917391463 & 1.663690914972 \\
\hline $\mathrm{O}$ & -1.807017658294 & 3.023605522288 & -0.003226301176 \\
\hline $\mathrm{H}$ & -4.144117382283 & 1.758641107667 & -1.959862413591 \\
\hline $\mathrm{C}$ & -4.349562422346 & 0.953295995156 & -1.266225700855 \\
\hline $\mathrm{C}$ & -3.291461003098 & 0.055246611289 & -0.959833516924 \\
\hline $\mathrm{C}$ & -5.569668779051 & 0.803244802843 & -0.686947799316 \\
\hline $\mathrm{C}$ & -3.514840751661 & -1.021795487637 & -0.056916758581 \\
\hline $\mathrm{C}$ & -2.027245096409 & 0.224406113829 & -1.508030036085 \\
\hline $\mathrm{C}$ & -5.774414411867 & -0.249916216808 & 0.236096161270 \\
\hline $\mathrm{H}$ & -6.378247835003 & 1.485720177940 & -0.910609010770 \\
\hline $\mathrm{C}$ & -4.788823394568 & -1.143259743128 & 0.549107125214 \\
\hline $\mathrm{N}$ & -2.498273899426 & -1.893827613834 & 0.213239787851 \\
\hline $\mathrm{C}$ & -0.982349461683 & -0.618257381982 & -1.158375743471 \\
\hline $\mathrm{H}$ & -6.738480657290 & -0.349027622218 & 0.718630622818 \\
\hline $\mathrm{H}$ & -4.989210935034 & -1.909187614613 & 1.280795142095 \\
\hline $\mathrm{C}$ & -1.233889062647 & -1.695239892853 & -0.260326612021 \\
\hline $\mathrm{C}$ & -2.773353051897 & -3.081470384779 & 1.034791582168 \\
\hline $\mathrm{C}$ & 0.330955967230 & -0.412728147217 & -1.665472999261 \\
\hline $\mathrm{C}$ & -0.150406727583 & -2.520858420737 & 0.127548991811 \\
\hline $\mathrm{H}$ & -2.139362302324 & -3.895698463083 & 0.706454623965 \\
\hline $\mathrm{H}$ & -2.592752321686 & -2.861099193291 & 2.086542200434 \\
\hline $\mathrm{H}$ & -3.800789103135 & -3.387951571057 & 0.884598750315 \\
\hline
\end{tabular}




\begin{tabular}{|c|c|c|c|}
\hline $\mathrm{C}$ & 1.351171529380 & -1.227911444156 & -1.288502941295 \\
\hline $\mathrm{H}$ & 0.484810461575 & 0.414676006261 & -2.347850486887 \\
\hline $\mathrm{C}$ & 1.096281699169 & -2.281584711223 & -0.378342753634 \\
\hline $\mathrm{H}$ & -0.281136826278 & -3.313534179731 & 0.846548845038 \\
\hline $\mathrm{H}$ & 2.354553715219 & -1.075814213116 & -1.664220860787 \\
\hline $\mathrm{H}$ & 1.917809644650 & -2.911825693600 & -0.060840393993 \\
\hline $\mathrm{H}$ & -1.849065272888 & 1.037743920045 & -2.203363067954 \\
\hline $\mathrm{H}$ & -0.256552554284 & -0.576471567363 & 3.091071067811 \\
\hline $\mathrm{N}$ & 0.035560262715 & 3.265855038156 & -1.275124224275 \\
\hline $\mathrm{H}$ & 0.930474438771 & 2.908687857627 & -1.565491847927 \\
\hline $\mathrm{H}$ & -0.505404978585 & 3.780875949139 & -1.952482025811 \\
\hline $\mathrm{H}$ & 2.087807965277 & 2.557810616730 & 0.127221535130 \\
\hline $\mathrm{H}$ & 2.172979544098 & -0.480651139194 & 2.929815924927 \\
\hline $\mathrm{C}$ & 3.673661314168 & 1.144095028612 & 1.556876521096 \\
\hline $\mathrm{C}$ & 4.167148090750 & 0.255787006539 & 0.438876515816 \\
\hline $\mathrm{H}$ & 4.059967832282 & 0.793956931386 & 2.513682623934 \\
\hline $\mathrm{H}$ & 4.026231420560 & 2.163914033689 & 1.403192007012 \\
\hline & 4.312321569221 & -1.115896824200 & 0.635752705263 \\
\hline $\mathrm{C}$ & 4.468526109545 & 0.795179348390 & -0.809662106741 \\
\hline $\mathrm{C}$ & 4.751494691747 & -1.934566196593 & -0.396749050510 \\
\hline $\mathrm{H}$ & 4.090024859535 & -1.542228187205 & 1.608065874485 \\
\hline $\mathrm{C}$ & 4.909202921640 & -0.021014520966 & -1.844590460389 \\
\hline $\mathrm{H}$ & 4.370074403776 & 1.863821244829 & -0.966248495616 \\
\hline & 5.052037293503 & -1.388025329605 & -1.639232043690 \\
\hline $\mathrm{H}$ & 4.866074369171 & -2.998665458004 & -0.230081700724 \\
\hline $\mathrm{H}$ & 5.146957759129 & 0.410961674475 & -2.808826196016 \\
\hline $\mathrm{H}$ & 5.400270761710 & -2.024685168460 & -2.442968459551 \\
\hline & & & \\
\hline $\mathrm{H}$ & 0.126887145019 & -0.168875366186 & -1.242658914935 \\
\hline $\mathrm{C}$ & 0.049955111229 & 0.910918944813 & -1.396668089829 \\
\hline & -1.309467698233 & 1.413696077380 & -0.995633768390 \\
\hline & 0.208988775827 & 1.049859752301 & -2.473750388576 \\
\hline & 1.123680101657 & 1.623365694041 & -0.606663625006 \\
\hline $\mathrm{C}$ & -1.479236117338 & 2.515368489207 & -0.266627384054 \\
\hline $\mathrm{C}$ & 0.845224761926 & 2.728500689483 & 0.114150295783 \\
\hline & 2.483621340105 & 1.063724813708 & -0.712992102132 \\
\hline & -0.406546296982 & 3.265569033244 & 0.219986247603 \\
\hline $\mathrm{O}$ & 2.686878934258 & 0.064329859629 & 37456270 \\
\hline $\mathrm{H}$ & -2.893266471482 & -3.105295047728 & -0.859289180090 \\
\hline & -2.767146473081 & -2.350906195846 & -0.092822843870 \\
\hline & -1.443534806647 & -2.048029014483 & 0.330853437487 \\
\hline & -3.831513306705 & -1.716045230188 & 0.465237763651 \\
\hline C & -1.230816492643 & -1.046376432533 & 1.318746330609 \\
\hline $\mathrm{C}$ & -0.345746915734 & -2.716860861477 & -0.194740325615 \\
\hline C & -3.613670421432 & -0.757704220800 & 1.484519209609 \\
\hline 1 & -4.840661738783 & -1.945512029821 & 0.151089644095 \\
\hline C & -2.358479433504 & -0.420148256713 & 1.904771502256 \\
\hline $\mathrm{N}$ & 0.042818896332 & -0.722912417396 & 1.685660098217 \\
\hline $\mathrm{C}$ & 0.937332140185 & -2.443644533860 & 0.256767858904 \\
\hline & -4.465896014085 & -0.278753090586 & 1.949911983061 \\
\hline & -2.244280875187 & 0.302486995601 & 2.697868101610 \\
\hline $\mathrm{C}$ & 1.123959506594 & -1.424801380057 & 1.235574703625 \\
\hline $\mathrm{C}$ & 0.245854455891 & 0.414025014099 & 2.596302076854 \\
\hline $\mathrm{C}$ & 2.059746403360 & -3.163991170102 & -0.236412991704 \\
\hline $\mathrm{C}$ & 2.429608466157 & -1.192356429926 & 1.729629261847 \\
\hline & 1.211299687779 & 0.863734813866 & 2.399205011259 \\
\hline & -0.511425278902 & 1.164224497787 & 2.394578013315 \\
\hline $\mathrm{H}$ & 0.184568599951 & 0.078902698001 & 3.631705673948 \\
\hline $\mathrm{C}$ & 3.305422904896 & -2.905979246414 & 0.239051875708 \\
\hline & 1.883399824564 & -3.921248754675 & -0.990079152397 \\
\hline $\mathrm{C}$ & 3.476738725861 & -1.918555975318 & 1.237939983751 \\
\hline
\end{tabular}




\begin{tabular}{|c|c|c|c|}
\hline $\mathrm{H}$ & 2.612982503199 & -0.470661014266 & 2.509136731770 \\
\hline $\mathrm{H}$ & 4.163709055855 & -3.452382009819 & -0.127900438025 \\
\hline $\mathrm{H}$ & 4.467901835192 & -1.734843038903 & 1.633516169614 \\
\hline $\mathrm{H}$ & -0.495337862533 & -3.474626086609 & -0.956084182686 \\
\hline $\mathrm{H}$ & -2.187515599863 & 0.879670103416 & -1.337742991981 \\
\hline $\mathrm{N}$ & 3.502192968092 & 1.646288232914 & -0.022321211205 \\
\hline $\mathrm{H}$ & 3.425990969746 & 2.568110119311 & 0.373446069303 \\
\hline $\mathrm{H}$ & 4.432753630698 & 1.313416499637 & -0.223799394113 \\
\hline $\mathrm{H}$ & -2.456904521060 & 2.898699919940 & -0.004501538225 \\
\hline $\mathrm{H}$ & 1.602575728174 & 3.269504495817 & 0.669030013839 \\
\hline $\mathrm{C}$ & -0.662903031446 & 4.338310388204 & 1.180494282364 \\
\hline $\mathrm{C}$ & -0.849036663789 & 3.809857736412 & 2.582030544631 \\
\hline $\mathrm{H}$ & -1.555111265267 & 4.869065291117 & 0.848810636486 \\
\hline $\mathrm{H}$ & 0.179881455005 & 5.028076737213 & 1.143195317972 \\
\hline $\mathrm{C}$ & -2.090505083542 & 3.332567791482 & 2.997010409814 \\
\hline $\mathrm{C}$ & 0.238078495769 & 3.712206988167 & 3.447296613583 \\
\hline $\mathrm{C}$ & -2.238633738129 & 2.748818497396 & 4.249140454229 \\
\hline $\mathrm{H}$ & -2.944576153382 & 3.416210681457 & 2.333843698025 \\
\hline $\mathrm{C}$ & 0.092696120958 & 3.129943324411 & 4.700083280630 \\
\hline $\mathrm{H}$ & 1.204616973463 & 4.092007071891 & 3.134490301292 \\
\hline $\mathrm{C}$ & -1.145523833244 & 2.641498292335 & 5.100743766551 \\
\hline $\mathrm{H}$ & -3.207996426436 & 2.379364225078 & 4.560835890658 \\
\hline $\mathrm{H}$ & 0.945071076263 & 3.058492117935 & 5.364410331760 \\
\hline $\mathrm{H}$ & -1.259881344576 & 2.186006676410 & 6.076474023389 \\
\hline & & & \\
\hline $\mathrm{H}$ & -1.490650797188 & -2.290757271607 & 0.413620725811 \\
\hline $\mathrm{C}$ & -0.422185665609 & -2.180761146816 & 0.632683357394 \\
\hline $\mathrm{C}$ & 0.394708175571 & -2.418337758409 & -0.606757714903 \\
\hline $\mathrm{H}$ & -0.212795920047 & -2.950418581089 & 1.385071058708 \\
\hline $\mathrm{C}$ & -0.144797939922 & -0.802136718756 & 1.191466745672 \\
\hline $\mathrm{C}$ & 1.295059171426 & -1.548540427932 & -1.061102245845 \\
\hline $\mathrm{C}$ & 0.795704964690 & 0.004783355876 & 0.656086850783 \\
\hline $\mathrm{C}$ & -0.990748835179 & -0.383575746019 & 2.320852414945 \\
\hline $\mathrm{N}$ & 1.532111882011 & -0.319907479930 & -0.443175770844 \\
\hline $\mathrm{O}$ & -1.911916008167 & -1.1057 & 2.703342761416 \\
\hline $\mathrm{H}$ & 0.397153643665 & 1.938686694638 & -2.749999153091 \\
\hline $\mathrm{C}$ & -0.109973266078 & 2.262850101806 & -1.849425808480 \\
\hline $\mathrm{C}$ & -1.167906251425 & 1.450267191438 & -1.356936713623 \\
\hline $\mathrm{C}$ & 0.254269605050 & 3.394408139886 & -1.192167979330 \\
\hline $\mathrm{C}$ & -1.869576132839 & 1.827903818358 & -0.174383489394 \\
\hline $\mathrm{C}$ & -1.494046911387 & 0.260231538384 & -1.990536655557 \\
\hline $\mathrm{C}$ & -0.434046983163 & 3.756325013039 & -0.009402615530 \\
\hline $\mathrm{H}$ & 1.064421202341 & 4.012703816421 & -1.554537782467 \\
\hline $\mathrm{C}$ & -1.464613213925 & 3.011692430822 & 0.491307235649 \\
\hline $\mathrm{N}$ & -2.874773421682 & 1.026897774271 & 0.288166104683 \\
\hline $\mathrm{C}$ & -2.483305073447 & -0.567399677478 & -1.480717263657 \\
\hline $\mathrm{H}$ & -0.134265223510 & 4.650004328325 & 0.523299789898 \\
\hline $\mathrm{H}$ & -1.932114931061 & 3.332902792344 & 1.406841911100 \\
\hline $\mathrm{C}$ & -3.176261908146 & -0.165569895304 & -0.306630978226 \\
\hline $\mathrm{C}$ & -3.691810918985 & 1.420798734484 & 1.446296670571 \\
\hline $\mathrm{C}$ & -2.796224772742 & -1.809317437400 & -2.098478132925 \\
\hline $\mathrm{C}$ & -4.165865488953 & -1.027964968282 & 0.225684187816 \\
\hline $\mathrm{H}$ & -4.740457584080 & 1.360857712751 & 1.164427810414 \\
\hline $\mathrm{H}$ & -3.486959034672 & 0.747392663007 & 2.277599623427 \\
\hline $\mathrm{H}$ & -3.477164364371 & 2.438537667327 & 1.731390318794 \\
\hline $\mathrm{C}$ & -3.753453669935 & -2.617235499265 & -1.571691952641 \\
\hline $\mathrm{H}$ & -2.244660239762 & -2.084256152860 & -2.989050793007 \\
\hline $\mathrm{C}$ & -4.434920439231 & -2.213518353339 & -0.397442096498 \\
\hline $\mathrm{H}$ & -4.697023437753 & -0.773168363165 & 1.128241315592 \\
\hline $\mathrm{H}$ & -3.995725076112 & -3.564598776326 & -2.033926500808 \\
\hline $\mathrm{H}$ & -5.186969123034 & -2.866591453833 & 0.026749160149 \\
\hline
\end{tabular}




\begin{tabular}{|c|c|c|c|}
\hline $\mathrm{H}$ & -0.959815914552 & -0.031873655785 & -2.888226670609 \\
\hline $\mathrm{H}$ & 0.245167430245 & -3.337909282166 & -1.158436011553 \\
\hline $\mathrm{N}$ & -0.781355508043 & 0.823498279739 & 2.911191368418 \\
\hline $\mathrm{H}$ & -1.307090147616 & 1.015068312329 & 3.750691184047 \\
\hline $\mathrm{H}$ & 0.058655112583 & 1.359130395966 & 2.770251806634 \\
\hline $\mathrm{H}$ & 1.889861602316 & -1.728845402905 & -1.947622715055 \\
\hline $\mathrm{H}$ & 1.009643300373 & 0.994112714657 & 1.045758453368 \\
\hline $\mathrm{C}$ & 2.670324218358 & 0.476621445698 & -0.852751265734 \\
\hline $\mathrm{C}$ & 3.999335987202 & -0.023623771316 & -0.324138344101 \\
\hline $\mathrm{H}$ & 2.503270469004 & 1.501095181598 & -0.513791257804 \\
\hline $\mathrm{H}$ & 2.701830895947 & 0.503595578866 & -1.944241092269 \\
\hline $\mathrm{C}$ & 5.175887572777 & 0.494249536736 & -0.863870867492 \\
\hline $\mathrm{C}$ & 4.080888163405 & -0.966030250019 & 0.694809345483 \\
\hline $\mathrm{C}$ & 6.411976628991 & 0.083042037392 & -0.388473654517 \\
\hline $\mathrm{H}$ & 5.118036204202 & 1.223896145035 & -1.664532081429 \\
\hline $\mathrm{C}$ & 5.320840891605 & -1.381583854745 & 1.170819085048 \\
\hline $\mathrm{H}$ & 3.176213978016 & -1.383964154437 & 1.120976210806 \\
\hline $\mathrm{C}$ & 6.487981159694 & -0.858414476801 & 0.633209705898 \\
\hline $\mathrm{H}$ & 7.317928632150 & 0.493495787600 & -0.817102902592 \\
\hline $\mathrm{H}$ & 5.371067960333 & -2.117632255237 & 1.963738914401 \\
\hline $\mathrm{H}$ & 7.452197974268 & -1.183666669540 & 1.003101623688 \\
\hline & TAH: $\quad$ G30a & & \\
\hline $\mathrm{H}$ & -1.685260036202 & 0.242060801476 & 1.438446316804 \\
\hline $\mathrm{C}$ & -0.854463697659 & 131756727 & 2.132673438994 \\
\hline $\mathrm{C}$ & -0.171967296768 & -0.887082798691 & 2.447260021712 \\
\hline $\mathrm{H}$ & -1.324412203421 & 0.829947161904 & 3.036226964868 \\
\hline $\mathrm{C}$ & 0.143466807470 & 1.395573689659 & 1.550531461677 \\
\hline $\mathrm{C}$ & 1.151681006351 & -1.040447860603 & 2.403991113346 \\
\hline $\mathrm{C}$ & 1.466589199164 & 1.139649358050 & 1.553003134429 \\
\hline $\mathrm{C}$ & -0.287575019925 & 2.687290344564 & 0.984171027727 \\
\hline $\mathrm{N}$ & 2.013831938372 & 03562668 & 2.023189377134 \\
\hline $\mathrm{O}$ & 0.488701971851 & 3.533854463316 & 0.544443531078 \\
\hline $\mathrm{H}$ & 1.084748467042 & -1.956856836451 & -0.966241134758 \\
\hline $\mathrm{C}$ & 0.649505882515 & -1.010904631088 & -1.265292148899 \\
\hline $\mathrm{C}$ & -0.751532942413 & -0.838996284181 & -1.094100532425 \\
\hline $\mathrm{C}$ & 1.408904045962 & -0.004979603595 & -1.773229006572 \\
\hline $\mathrm{C}$ & -1.368496090066 & 0.384779486953 & -1.482816731785 \\
\hline $\mathrm{C}$ & -1.530336167717 & -1.837524915653 & -0.527384283605 \\
\hline $\mathrm{C}$ & 0.793392872280 & 1.221961226858 & -2.119967988970 \\
\hline $\mathrm{H}$ & 2.477020189765 & -0.125670226334 & -1.897730562785 \\
\hline $\mathrm{C}$ & -0.551833065021 & 1.423163614540 & -1.989939973774 \\
\hline $\mathrm{N}$ & -2.719532380791 & 0.529349959433 & -1.340463190823 \\
\hline $\mathrm{C}$ & -2.882538917632 & -1.634182545109 & -0.290100173671 \\
\hline $\mathrm{H}$ & 1.407579778705 & 2.034754149437 & -2.486811969938 \\
\hline $\mathrm{H}$ & -0.964236790643 & 2.390029215993 & -2.231215290120 \\
\hline $\mathrm{C}$ & -3.477545732556 & -0.405459442443 & -0.696853633381 \\
\hline $\mathrm{C}$ & -3.379475015076 & 1.717739160138 & -1.899619142738 \\
\hline $\mathrm{C}$ & -3.673163637252 & -2.620335524557 & 0.362163675915 \\
\hline $\mathrm{C}$ & -4.844227945242 & -0.184067716122 & -0.400891412585 \\
\hline $\mathrm{H}$ & -4.370564140603 & 1.443483895465 & -2.242111835421 \\
\hline $\mathrm{H}$ & -3.441999169000 & 2.504399698038 & -1.148442252736 \\
\hline $\mathrm{H}$ & -2.820428052864 & 2.063728758313 & -2.759852793776 \\
\hline $\mathrm{C}$ & -4.987074147523 & -2.389452073183 & 0.620590657297 \\
\hline $\mathrm{H}$ & -3.190718317984 & -3.546489692776 & 0.649014567868 \\
\hline $\mathrm{C}$ & -5.562567191133 & -1.152717300878 & 0.241921224990 \\
\hline $\mathrm{H}$ & -5.321320745804 & 0.752048265449 & -0.643595860222 \\
\hline $\mathrm{H}$ & -5.592945733595 & -3.132416850014 & 1.121094988405 \\
\hline $\mathrm{H}$ & -6.602521014234 & -0.961655659291 & 0.474491892990 \\
\hline $\mathrm{H}$ & -1.070479611479 & -2.777012774864 & -0.240293532540 \\
\hline $\mathrm{H}$ & -0.779175109425 & -1.732143240829 & 2.746163109150 \\
\hline $\mathrm{N}$ & -1.629082710701 & 2.891939015926 & 0.957151548421 \\
\hline
\end{tabular}




\begin{tabular}{|c|c|c|c|}
\hline $\mathrm{H}$ & -1.967961607487 & 3.790733427714 & 0.649782264986 \\
\hline $\mathrm{H}$ & -2.278918617750 & 2.271733195985 & 1.411652171380 \\
\hline $\mathrm{H}$ & 1.637576705903 & -1.972695431069 & 2.660869197844 \\
\hline $\mathrm{H}$ & 2.163558241801 & 1.870476489695 & 1.159561578697 \\
\hline $\mathrm{C}$ & 3.459580145057 & -0.212339937024 & 2.000191952448 \\
\hline $\mathrm{C}$ & 4.001004612174 & -0.555165198137 & 0.632492997311 \\
\hline $\mathrm{H}$ & 3.686523805689 & -1.012480609266 & 2.704533545108 \\
\hline $\mathrm{H}$ & 3.934056851808 & 0.698936783003 & 2.365175987704 \\
\hline $\mathrm{C}$ & 4.701104863861 & 0.391828174193 & -0.108047362842 \\
\hline $\mathrm{C}$ & 3.797488144296 & -1.823619446809 & 0.090884288238 \\
\hline $\mathrm{C}$ & 5.194710093061 & 0.079080082877 & -1.370421590839 \\
\hline $\mathrm{H}$ & 4.866783000570 & 1.378467418553 & 0.310281633577 \\
\hline $\mathrm{C}$ & 4.284790165069 & -2.138204671504 & -1.169578335295 \\
\hline $\mathrm{H}$ & 3.257695174553 & -2.569034891951 & 0.665422756511 \\
\hline $\mathrm{C}$ & 4.987402011366 & -1.186267862601 & -1.902842613410 \\
\hline $\mathrm{H}$ & 5.741419236495 & 0.823816943282 & -1.935510910958 \\
\hline $\mathrm{H}$ & 4.123980618167 & -3.127737376368 & -1.579562139801 \\
\hline $\mathrm{H}$ & 5.371982198621 & -1.433036933481 & -2.884550751938 \\
\hline \multicolumn{4}{|c|}{$\mathrm{MA}+$ BNAH: $\quad$ G30 } \\
\hline $\mathrm{H}$ & -0.009077881905 & -0.535545975951 & 0.181892019442 \\
\hline $\mathrm{C}$ & 0.326246585708 & 0.186642988758 & 0.931706217749 \\
\hline $\mathrm{C}$ & 1.678353040447 & 0.743364081958 & 0.576254152574 \\
\hline $\mathrm{H}$ & 0.380612409918 & -0.388168774476 & 1.865253847403 \\
\hline $\mathrm{C}$ & -0.683081471945 & 1.305111871696 & 1.060173036151 \\
\hline $\mathrm{C}$ & 1.953114963055 & 2.046803141534 & 0.618724450952 \\
\hline $\mathrm{C}$ & -0.303437735816 & 2.599161359611 & 1.071521079202 \\
\hline $\mathrm{C}$ & -2.097694835718 & 0.904576858774 & 1.148184154965 \\
\hline $\mathrm{N}$ & 0.993192971691 & 3.004196032130 & 0.942082228373 \\
\hline $\mathrm{O}$ & -2.434305030709 & -0.253436193279 & 0.900635287665 \\
\hline $\mathrm{H}$ & 1.044392966917 & 2.504820209096 & -2.744249359388 \\
\hline $\mathrm{C}$ & 0.027258563615 & 2.192837460875 & -2.539590384433 \\
\hline $\mathrm{C}$ & -0.236644035298 & 0.798476156631 & -2.448359960003 \\
\hline $\mathrm{C}$ & -0.972482222908 & 3.095233164866 & -2.359281202539 \\
\hline $\mathrm{C}$ & -1.559810553430 & 0.344020006181 & -2.187650527194 \\
\hline $\mathrm{C}$ & 0.780243802133 & -0.135254323908 & -2.587235109889 \\
\hline $\mathrm{C}$ & -2.280037030684 & 2.635291007408 & -2.068180847323 \\
\hline $\mathrm{H}$ & -0.777295935634 & 4.157599129177 & -2.420878243492 \\
\hline $\mathrm{C}$ & -2.580291318689 & 1.305170671964 & -1.986988454836 \\
\hline $\mathrm{N}$ & -1.811738592619 & -0.995307964776 & -2.120710809088 \\
\hline $\mathrm{C}$ & 0.526859738350 & -1.489052105183 & -2.420786189245 \\
\hline $\mathrm{H}$ & -3.066442146428 & 3.358691650885 & -1.891351032155 \\
\hline $\mathrm{H}$ & -3.582524862997 & 1.005430190169 & -1.724590146066 \\
\hline $\mathrm{C}$ & -0.807632492620 & -1.917127972357 & -2.158699475797 \\
\hline $\mathrm{C}$ & -3.205850018393 & -1.440764765852 & -1.987291604894 \\
\hline $\mathrm{C}$ & 1.577746169470 & -2.446081773322 & -2.485182007460 \\
\hline $\mathrm{C}$ & -1.039661596820 & -3.294740034056 & -1.922362280451 \\
\hline $\mathrm{H}$ & -3.305908300294 & -2.438529171305 & -2.393618201951 \\
\hline $\mathrm{H}$ & -3.497397721512 & -1.414543279806 & -0.938177576680 \\
\hline $\mathrm{H}$ & -3.838997672288 & -0.784473222191 & -2.574119869116 \\
\hline $\mathrm{C}$ & 1.323818227714 & -3.763564129456 & -2.273756316819 \\
\hline $\mathrm{H}$ & 2.576827589697 & -2.085610975053 & -2.696498201285 \\
\hline $\mathrm{C}$ & 0.002080792069 & -4.176543442339 & -1.977727446741 \\
\hline $\mathrm{H}$ & -2.020315544989 & -3.659060866668 & -1.662565032797 \\
\hline $\mathrm{H}$ & 2.118525460326 & -4.495920266635 & -2.315130924829 \\
\hline $\mathrm{H}$ & -0.191438199753 & -5.223132486137 & -1.779486174411 \\
\hline $\mathrm{H}$ & 1.789795574451 & 0.199412148860 & -2.799961485095 \\
\hline $\mathrm{H}$ & 2.470152994282 & 0.058360579922 & 0.299903972752 \\
\hline $\mathrm{N}$ & -3.027794024610 & 1.837563657024 & 1.481453388957 \\
\hline $\mathrm{H}$ & -3.971793070106 & 1.511407980225 & 1.622243183296 \\
\hline $\mathrm{H}$ & -2.772351528020 & 2.704123087717 & 1.925099048052 \\
\hline $\mathrm{H}$ & 2.934632311239 & 2.445135150832 & 0.397160698109 \\
\hline
\end{tabular}




$\begin{array}{llll}\mathrm{H} & -1.015434822435 & 3.412384871312 & 1.159644032073 \\ \mathrm{C} & 1.333020662857 & 4.422544190887 & 0.994806444867 \\ \mathrm{C} & 1.023530643533 & 5.173651079430 & -0.278626101882 \\ \mathrm{H} & 2.397784275373 & 4.488564437536 & 1.217988096219 \\ \mathrm{H} & 0.790201754928 & 4.872299606697 & 1.826627214437 \\ \mathrm{C} & -0.063978989017 & 6.038990902647 & -0.341437465399 \\ \mathrm{C} & 1.822657052372 & 5.006910202565 & -1.408890988065 \\ \mathrm{C} & -0.350330629768 & 6.731971825026 & -1.513193693020 \\ \mathrm{H} & -0.685311461347 & 6.177693470936 & 0.536455618960 \\ \mathrm{C} & 1.538164764511 & 5.693391181981 & -2.580614011660 \\ \mathrm{H} & 2.676110526665 & 4.338563236072 & -1.365562561105 \\ \mathrm{C} & 0.450676144372 & 6.560634228580 & -2.634123493129 \\ \mathrm{H} & -1.197160118203 & 7.406152395527 & -1.548044011606 \\ \mathrm{H} & 2.167760429430 & 5.559298383111 & -3.451653512303 \\ \mathrm{H} & 0.231728029828 & 7.100541474150 & -3.546856280050\end{array}$

\begin{tabular}{|c|c|c|c|}
\hline \multicolumn{4}{|c|}{ MA+ BNAH: $\quad$ G33 } \\
\hline $\mathrm{H}$ & 1.643444602363 & -2.121897886364 & -1.105971003935 \\
\hline $\mathrm{C}$ & 0.556132017045 & -2.059587968453 & -1.238761511038 \\
\hline $\mathrm{C}$ & -0.153243437749 & -2.542924381121 & -0.004514937400 \\
\hline $\mathrm{H}$ & 0.342207068799 & -2.717923664823 & -2.087906386467 \\
\hline $\mathrm{C}$ & 0.150178529818 & -0.638444702221 & -1.559161930582 \\
\hline $\mathrm{C}$ & -1.003959979060 & -1.788213487706 & 0.687595026407 \\
\hline $\mathrm{C}$ & -0.721752462277 & 0.043442694950 & -0.789183260288 \\
\hline $\mathrm{C}$ & 0.805591502226 & -0.031198833398 & -2.731423135331 \\
\hline $\mathrm{N}$ & -1.294890369303 & -0.466427520775 & 0.340409682846 \\
\hline $\mathrm{O}$ & 1.670304949454 & -0.652952599663 & -3.345406865295 \\
\hline $\mathrm{H}$ & 4.593228969799 & -0.301056957927 & -1.835513655266 \\
\hline $\mathrm{C}$ & 4.192629949488 & -0.729150127351 & -0.925343096422 \\
\hline $\mathrm{C}$ & 3.212956366586 & 0.020907692602 & -0.216560992691 \\
\hline $\mathrm{C}$ & 4.603169709391 & -1.938171635411 & -0.462894385930 \\
\hline $\mathrm{C}$ & 2.635008156827 & 463394958 & 0.9749804 \\
\hline $\mathrm{C}$ & 2.801388585477 & 1.262214595381 & -0.676320744988 \\
\hline $\mathrm{C}$ & 4.051337155920 & -2.440272542790 & 0.740142812921 \\
\hline $\mathrm{H}$ & 5.348065176976 & -2.513926722873 & -0.994878677407 \\
\hline $\mathrm{C}$ & 3.099969322923 & -1.759458235533 & 1.446474821911 \\
\hline $\mathrm{N}$ & 1.666725613684 & 4491 & 99536462 \\
\hline $\mathrm{C}$ & 1.856039187314 & 2.000922175743 & 0.021066705881 \\
\hline $\mathrm{H}$ & 4.396361006893 & -3.393148236565 & 1.121112305468 \\
\hline $\mathrm{H}$ & 2.739869365725 & -2.183824001991 & 2.369170902663 \\
\hline $\mathrm{C}$ & 1.291628399533 & & 1.206242708932 \\
\hline $\mathrm{C}$ & 1.015865560813 & 059572368 & 2.817010517724 \\
\hline $\mathrm{C}$ & 1.427034682504 & 3.272249308245 & -0.447519279690 \\
\hline $\mathrm{C}$ & 0.340772925201 & 2.223383145474 & 1.919422112826 \\
\hline $\mathrm{H}$ & 1.476116694238 & 0.068237980867 & 3.712729556627 \\
\hline $\mathrm{H}$ & -0.040414712391 & 019481935 & 20026355 \\
\hline $\mathrm{H}$ & 1.098500423370 & -1.424980857356 & 2.816314150021 \\
\hline $\mathrm{C}$ & 0.491293506033 & 3.979679084585 & 0.239760225866 \\
\hline $\mathrm{H}$ & 1.867896939071 & 3.648985607427 & -1.361945959547 \\
\hline $\mathrm{C}$ & -0.038920575464 & 3.445431340765 & 1.438202533781 \\
\hline $\mathrm{H}$ & -0.079953372299 & & 2.848395412319 \\
\hline $\mathrm{H}$ & 0.158321236612 & 4.947221428223 & -0.110438242010 \\
\hline $\mathrm{H}$ & -0.765691367382 & 4.021400459641 & 1.997035765578 \\
\hline $\mathrm{H}$ & 3.223956766604 & 1.660997643194 & -1.592333364904 \\
\hline $\mathrm{H}$ & 0.039180080345 & -3.549931832328 & 0.343592578450 \\
\hline $\mathrm{N}$ & 0.472575823112 & 1.233888111378 & -3.102304892629 \\
\hline $\mathrm{H}$ & -0.348711893484 & 1.699953334283 & -2.755838583369 \\
\hline $\mathrm{H}$ & 0.858975902018 & 1.571977300362 & -3.970327294510 \\
\hline $\mathrm{H}$ & -1.012233403460 & 1.066947615782 & -1.001284522983 \\
\hline $\mathrm{H}$ & -1.513424904054 & -2.142157128635 & 1.575163909897 \\
\hline $\mathrm{C}$ & -2.397676122456 & 0.210324288877 & 0.993656010701 \\
\hline $\mathrm{C}$ & -3.766734987103 & -0.258043019352 & 0.543279161590 \\
\hline
\end{tabular}




$\begin{array}{llll}\mathrm{H} & -2.292586730884 & 1.280528734915 & 0.803465017205 \\ \mathrm{H} & -2.304418687029 & 0.067272102586 & 2.073056220865 \\ \mathrm{C} & -3.945727514507 & -0.992487460263 & -0.623970034433 \\ \mathrm{C} & -4.881275961777 & 0.074270327954 & 1.311731850959 \\ \mathrm{C} & -5.220776705230 & -1.383349949252 & -1.020602238876 \\ \mathrm{H} & -3.089769588745 & -1.267487331642 & -1.229343572604 \\ \mathrm{C} & -6.153076606615 & -0.312402054236 & 0.916046635863 \\ \mathrm{H} & -4.747085278304 & 0.638255568732 & 2.228477552660 \\ \mathrm{C} & -6.326737113472 & -1.044035431673 & -0.254309983655 \\ \mathrm{H} & -5.346343825551 & -1.956464041898 & -1.930940824179 \\ \mathrm{H} & -7.009928099018 & -0.048112065596 & 1.523433878501 \\ \mathrm{H} & -7.318360478544 & -1.350432928221 & -0.562705729528\end{array}$

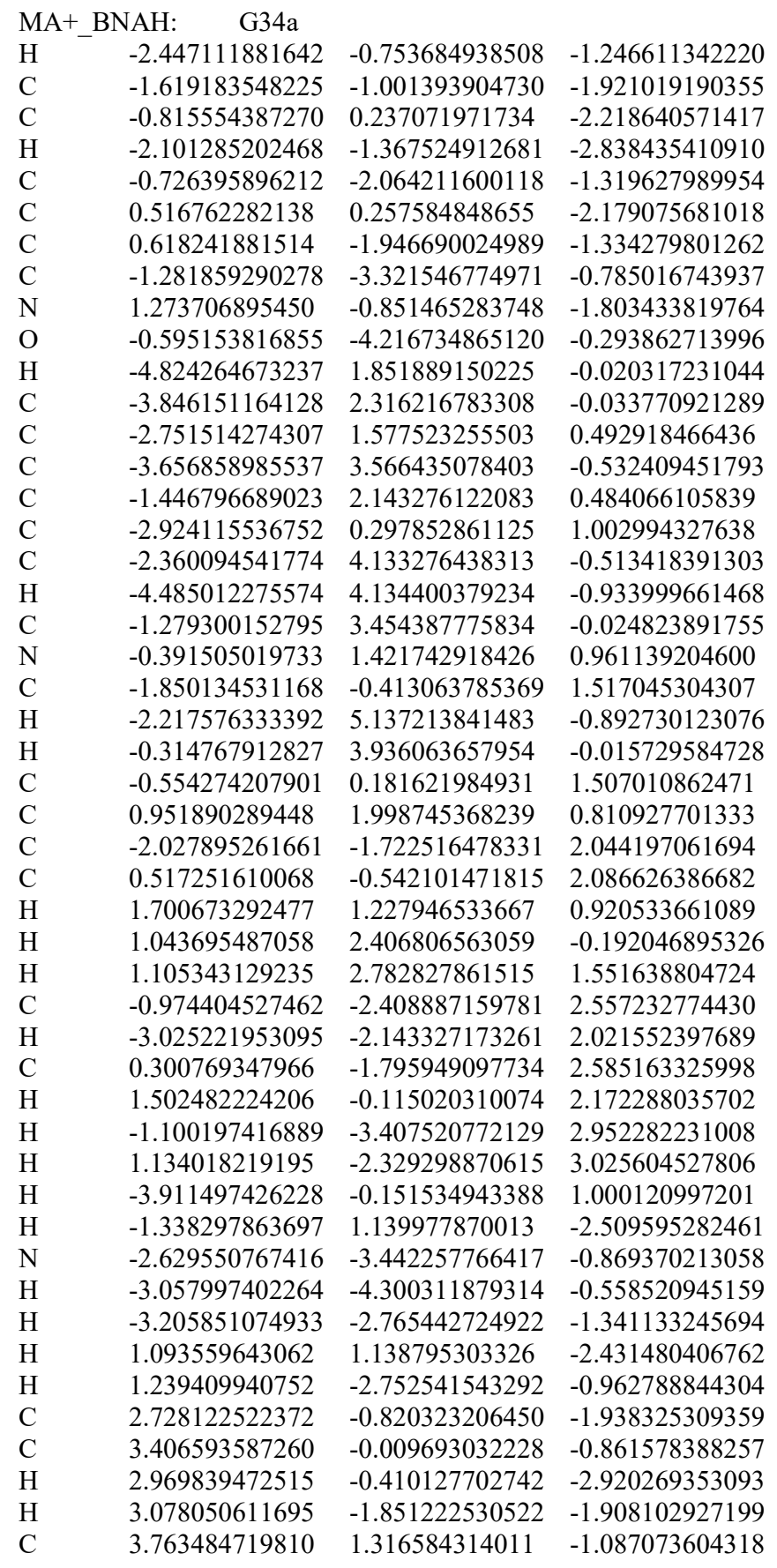




\begin{tabular}{|c|c|c|c|}
\hline $\mathrm{C}$ & 3.656782604538 & -0.576510091583 & 0.386683217892 \\
\hline $\mathrm{C}$ & 4.342913613107 & 2.073589746053 & -0.075431916559 \\
\hline $\mathrm{H}$ & 3.584624681347 & 1.758324560471 & -2.061229069277 \\
\hline $\mathrm{C}$ & 4.240770584025 & 0.174733452514 & 1.397911571355 \\
\hline $\mathrm{H}$ & 3.389843262740 & -1.612982612380 & 0.564866970412 \\
\hline $\mathrm{C}$ & 4.579155667997 & 1.504512936481 & 1.169798853665 \\
\hline $\mathrm{H}$ & 4.612841074449 & 3.105914581678 & -0.260657000593 \\
\hline $\mathrm{H}$ & 4.432619583720 & -0.276247489180 & 2.363863207799 \\
\hline $\mathrm{H}$ & 5.033689436598 & 2.091588798156 & 1.958003544937 \\
\hline \multicolumn{4}{|c|}{ MA+_BNAH: $\quad$ G34 } \\
\hline $\mathrm{H}$ & -2.454016755871 & -0.733702864893 & -1.349340930950 \\
\hline $\mathrm{C}$ & -1.606057058734 & -0.922264651523 & -2.011974623887 \\
\hline $\mathrm{C}$ & -0.804221492810 & 0.331706571576 & -2.229716408595 \\
\hline $\mathrm{H}$ & -2.059367561128 & -1.255612568666 & -2.954956630753 \\
\hline $\mathrm{C}$ & -0.730980467013 & -2.011442338445 & -1.434420368791 \\
\hline $\mathrm{C}$ & 0.527753304634 & 0.342360103233 & -2.190054859820 \\
\hline $\mathrm{C}$ & 0.615068503268 & -1.900692136042 & -1.432433282547 \\
\hline $\mathrm{C}$ & -1.432208297928 & -3.204201812149 & -0.927452961359 \\
\hline $\mathrm{N}$ & 1.274761447097 & -0.792567611044 & -1.874400132898 \\
\hline $\mathrm{O}$ & -2.659089515080 & -3.212909772043 & -0.847203699334 \\
\hline $\mathrm{H}$ & -4.822014581781 & 1.659338895780 & -0.009652748725 \\
\hline $\mathrm{C}$ & -3.866146579610 & 2.167578241735 & 0.010841174883 \\
\hline $\mathrm{C}$ & -2.746513563926 & 1.456689188265 & 0.523258947303 \\
\hline $\mathrm{C}$ & -3.727309879574 & 3.445362845089 & -0.431310900066 \\
\hline $\mathrm{C}$ & -1.467229497866 & 2.078148645414 & 0.552020502542 \\
\hline $\mathrm{C}$ & -2.870649857599 & 0.152217512151 & 0.982509084053 \\
\hline $\mathrm{C}$ & -2.458522215162 & 4.069279663967 & -0.365463674181 \\
\hline $\mathrm{H}$ & -4.574879441551 & 3.992425257475 & -0.821167113477 \\
\hline $\mathrm{C}$ & -1.353947495225 & 3.417864298507 & 0.106934172370 \\
\hline $\mathrm{N}$ & -0.386399480604 & 1.380208437100 & 1.006130051600 \\
\hline $\mathrm{C}$ & 45529898 & 26004 & 31930468 \\
\hline $\mathrm{H}$ & -2.357641983759 & 1212 & -0.693856363871 \\
\hline $\mathrm{H}$ & -0.413796673323 & 3.943320625607 & 0.157238585435 \\
\hline $\mathrm{C}$ & -0.506302701818 & 0.120759112083 & 1.516828254178 \\
\hline $\mathrm{C}$ & 0.942118555026 & 1.996039619206 & 53242 \\
\hline $\mathrm{C}$ & 63458371 & 869505041 & .54042 \\
\hline $\mathrm{C}$ & 0.588396306010 & 0589378 & 170887 \\
\hline $\mathrm{H}$ & 1.701990173688 & 1.226452171025 & 828892 \\
\hline $\mathrm{H}$ & 0.995912487271 & 2.507711483457 & -0.081540831741 \\
\hline $\mathrm{H}$ & 1.112716059098 & 923818131 & 72191 \\
\hline $\mathrm{C}$ & -0.828044221072 & -2.506911431004 & 323471364 \\
\hline $\mathrm{H}$ & -2.878678941201 & -2.321182204115 & 1.932758628160 \\
\hline $\mathrm{C}$ & 0.419526256321 & -1.841159218871 & 2.562414117168 \\
\hline $\mathrm{H}$ & 1.550351124532 & -0.096274019815 & 2.205070203527 \\
\hline $\mathrm{H}$ & -0.917339976060 & -3.517843263442 & 5792785 \\
\hline $\mathrm{H}$ & 1.267991347539 & -2.347449937181 & 3.005950515957 \\
\hline $\mathrm{H}$ & -3.836580785073 & -0.339817660307 & 0.946435056144 \\
\hline $\mathrm{H}$ & -1.323272194205 & 1.254236210632 & -2.460294121251 \\
\hline $\mathrm{N}$ & -0.708953566508 & -4.287243792919 & -0.534514420312 \\
\hline $\mathrm{H}$ & -1.229424175094 & -5.117411961277 & -0.295721704576 \\
\hline $\mathrm{H}$ & 0.255246719212 & -4.413326783620 & -0.792443645708 \\
\hline $\mathrm{H}$ & 1.113376724191 & 1.231530024459 & -2.388446789571 \\
\hline $\mathrm{H}$ & 1.266377645011 & -2.684227745532 & -1.064395272333 \\
\hline $\mathrm{C}$ & 2.729891502935 & -0.760301150339 & -2.000297658941 \\
\hline $\mathrm{C}$ & 3.407687211548 & 0.015062613532 & -0.897180117580 \\
\hline $\mathrm{H}$ & 2.976257497776 & -0.320063025641 & -2.968019899314 \\
\hline $\mathrm{H}$ & 3.079355371653 & -1.791849181432 & -2.002467801346 \\
\hline $\mathrm{C}$ & 3.772783403067 & 1.345543678557 & -1.082729237551 \\
\hline $\mathrm{C}$ & 3.651866493667 & -0.589260951108 & 0.334431733231 \\
\hline $\mathrm{C}$ & 4.354401539079 & 2.069135009258 & -0.048292285257 \\
\hline $\mathrm{H}$ & 3.599113773863 & 1.816511034296 & -2.044020151401 \\
\hline
\end{tabular}




\begin{tabular}{|c|c|c|c|}
\hline $\mathrm{C}$ & 4.238538526432 & 0.128487897652 & 1.368231018558 \\
\hline $\mathrm{H}$ & 3.379585789881 & -1.629122280146 & 0.481911829470 \\
\hline $\mathrm{C}$ & 4.584946507051 & 1.462285288450 & 1.180148543072 \\
\hline $\mathrm{H}$ & 4.630752895839 & 3.104793644592 & -0.202708377952 \\
\hline $\mathrm{H}$ & 4.425819434603 & -0.351961815026 & 2.320829974505 \\
\hline $\mathrm{H}$ & 5.041517160777 & 2.023130373018 & 1.986062452314 \\
\hline & NAH: $\quad$ G35a & & \\
\hline $\mathrm{H}$ & -1.857530965553 & 1.040597632658 & 1.194783432592 \\
\hline $\mathrm{C}$ & -1.048916872633 & 1.301327425666 & 1.888779983476 \\
\hline $\mathrm{C}$ & -0.457259249229 & 0.045718079385 & 2.470781414388 \\
\hline $\mathrm{H}$ & -1.533069965858 & 1.891032497484 & 2.679104687894 \\
\hline $\mathrm{C}$ & 0.026314638997 & 2.110552742301 & 1.195814578915 \\
\hline $\mathrm{C}$ & 0.850418187591 & -0.207470565598 & 2.471622673288 \\
\hline $\mathrm{C}$ & 1.328072956647 & 1.761591600994 & 1.257497222227 \\
\hline $\mathrm{C}$ & -0.301203218783 & 3.352232489622 & 0.470148739534 \\
\hline $\mathrm{N}$ & 1.786925755521 & 0.661608085242 & 1.913753166469 \\
\hline $\mathrm{O}$ & 0.540650036199 & 54168599 & 81798 \\
\hline $\mathrm{H}$ & -5.402165578624 & 0.151476867351 & -0.728508008971 \\
\hline $\mathrm{C}$ & -4.764707674019 & -0.662824540935 & -0.407391560265 \\
\hline $\mathrm{C}$ & -3.369404283975 & -0.552892697069 & -0.658280442146 \\
\hline $\mathrm{C}$ & -5.268790434514 & -1.764519243378 & 0.208053248623 \\
\hline $\mathrm{C}$ & -2.489929261556 & -1.591345704496 & 040983 \\
\hline $\mathrm{C}$ & -2.839041117029 & 0.554278088992 & -1.306152034637 \\
\hline $\mathrm{C}$ & -4.392545883850 & -2.808691966121 & 0.589257185688 \\
\hline $\mathrm{H}$ & -6.329294287140 & -1.857315318454 & 0.398748933865 \\
\hline $\mathrm{C}$ & -3.043814453016 & -2.737362284560 & 0.380948742507 \\
\hline $\mathrm{N}$ & -1.147702605326 & -1.468120394065 & 883341 \\
\hline $\mathrm{C}$ & -1.483139297340 & 0.631558340427 & -1.588551546486 \\
\hline $\mathrm{H}$ & -4.801333073027 & -3.696620543510 & 1.054571192846 \\
\hline $\mathrm{H}$ & -2.423057273671 & -3.571301469467 & 0.666461688268 \\
\hline $\mathrm{C}$ & -0.623631927582 & 17904196 & 99642079 \\
\hline $\mathrm{C}$ & -0.249548280417 & 6601247744 & 0.086453379803 \\
\hline $\mathrm{C}$ & -0.948445606314 & 1.743591321653 & -2.295081950058 \\
\hline $\mathrm{C}$ & 0.747264884686 & -0.358934036887 & -1.517231076465 \\
\hline $\mathrm{H}$ & 0.731859109998 & -2.069339747348 & 2389 \\
\hline $\mathrm{H}$ & 6271279 & 390445581 & 56094 \\
\hline $\mathrm{H}$ & -0.192061796008 & -3.341661021686 & -0.599724098402 \\
\hline $\mathrm{C}$ & 0.373058051108 & 1.791016889746 & -2.603531353931 \\
\hline $\mathrm{H}$ & -1.628887614641 & 2.537146993871 & -2.577319754315 \\
\hline $\mathrm{C}$ & 1.211571388415 & 0.718118676907 & 903960 \\
\hline $\mathrm{H}$ & 012416 & 50942276 & 24580 \\
\hline $\mathrm{H}$ & 0.788426559012 & 2.631973138614 & -3.141499542435 \\
\hline $\mathrm{H}$ & 2.258518371278 & 0.745076062997 & -2.494041609123 \\
\hline $\mathrm{H}$ & -3.494988300405 & 1.362880807518 & -1.610565073784 \\
\hline $\mathrm{H}$ & -1.122071547551 & -0.676456547049 & 2.928043674538 \\
\hline $\mathrm{N}$ & -1.619096480058 & 3.666867297812 & 0.423633609015 \\
\hline $\mathrm{H}$ & -1.899004996123 & 4.510916343041 & -0.051032898728 \\
\hline $\mathrm{H}$ & -2.329405263960 & 3.108453809448 & 0.866880332689 \\
\hline $\mathrm{H}$ & 1.267981014170 & -1.103268481823 & 2.912772097289 \\
\hline $\mathrm{H}$ & 2.079230574120 & 2.373925238973 & 0.773159926485 \\
\hline $\mathrm{C}$ & 3.199704922232 & 0.294650033942 & 1.890460401278 \\
\hline $\mathrm{C}$ & 3.549080441947 & -0.626526852117 & 0.746013500748 \\
\hline $\mathrm{H}$ & 3.432102504024 & -0.183868665901 & 2.842605486989 \\
\hline $\mathrm{H}$ & 3.779245774391 & 1.214428329563 & 1.821736665424 \\
\hline $\mathrm{C}$ & 3.321160078997 & -1.997683234266 & 0.847296723591 \\
\hline $\mathrm{C}$ & 4.064251378502 & -0.112939467900 & -0.440599165375 \\
\hline $\mathrm{C}$ & 3.580542623513 & -2.837694493988 & -0.227977172728 \\
\hline $\mathrm{H}$ & 2.937014419999 & -2.408711404253 & 1.774692816274 \\
\hline $\mathrm{C}$ & 4.334107061993 & -0.951474495814 & -1.515398521805 \\
\hline $\mathrm{H}$ & 4.252728671563 & 0.952135504982 & -0.522479488255 \\
\hline $\mathrm{C}$ & 4.085464236440 & -2.314757134271 & -1.413525302270 \\
\hline
\end{tabular}




\begin{tabular}{|c|c|c|c|}
\hline $\mathrm{H}$ & 3.394705296011 & -3.901088789004 & -0.139512367869 \\
\hline $\mathrm{H}$ & 4.736207301633 & -0.540089372524 & -2.433161182065 \\
\hline & 4.292984078077 & -2.969161625509 & -2.250932856335 \\
\hline & G35 & & \\
\hline $\mathrm{H}$ & -1.974730168448 & 0.837460221359 & 1.224955478926 \\
\hline $\mathrm{C}$ & -1.211237665812 & 1.085967866351 & 1.967916639060 \\
\hline $\mathrm{C}$ & -0.546037162273 & -0.158125472173 & 2.488224461307 \\
\hline $\mathrm{H}$ & -1.761978809194 & 1.590002722575 & 2.771938408144 \\
\hline $\mathrm{C}$ & -0.185549964226 & 2.021691825586 & 1.368190207374 \\
\hline $\mathrm{C}$ & 0.776138519973 & -0.314927757938 & 2.513109908690 \\
\hline $\mathrm{C}$ & 1.137400753622 & 1.759269360346 & 1.429847398802 \\
\hline $\mathrm{C}$ & -0.719245095829 & 3.229734199700 & 0.716277250050 \\
\hline $\mathrm{N}$ & 1.658505878877 & 0.652176280806 & 2.031482890173 \\
\hline $\mathrm{O}$ & -1.924581723595 & 3.336450683151 & 0.498431520648 \\
\hline $\mathrm{H}$ & -5.323937182627 & 0.010960652976 & -0.839018160092 \\
\hline $\mathrm{C}$ & -4.664602217954 & -0.801949493946 & -0.561551267776 \\
\hline $\mathrm{C}$ & -3.269155056629 & -0.625348356839 & -0.768995593151 \\
\hline $\mathrm{C}$ & -5.141852612797 & -1.962074721625 & -0.038742536426 \\
\hline $\mathrm{C}$ & -2.361854677754 & -1.659353760979 & -0.402261335801 \\
\hline $\mathrm{C}$ & -2.765800150152 & 0.54 & 67556 \\
\hline $\mathrm{C}$ & -4.236743725090 & -2.999416369470 & 0.289289678865 \\
\hline $\mathrm{H}$ & -6.202247222872 & -2.105240584020 & 0.118414331281 \\
\hline $\mathrm{C}$ & -2.887030157903 & -2.865689713225 & 0.121075133721 \\
\hline $\mathrm{N}$ & -1.020290164306 & -1.47218 & 32894 \\
\hline $\mathrm{C}$ & -1.408128397225 & 0.68 & 63873394 \\
\hline $\mathrm{H}$ & -4.622865661553 & -3.932366172395 & 0.679930087196 \\
\hline $\mathrm{H}$ & -2.242367909611 & -3.695492598332 & 0.362313111873 \\
\hline $\mathrm{C}$ & -0.520317183451 & -0.363339192892 & -1.194774319828 \\
\hline $\mathrm{C}$ & -0.094394131197 & -2.4982692 & 28423 \\
\hline $\mathrm{C}$ & -0.900251539118 & 1.86 & 4899 \\
\hline $\mathrm{C}$ & 0.855252901860 & -0.22 & 117928 \\
\hline $\mathrm{H}$ & 0.858805719631 & -2.039310086132 & 101894 \\
\hline $\mathrm{H}$ & -0.483476531757 & -2.910737981852 & 0.8501341 \\
\hline $\mathrm{H}$ & 0.029794755839 & -3.284237006306 & 36776 \\
\hline $\mathrm{C}$ & 0.42460 & 1.97 & 83401 \\
\hline $\mathrm{H}$ & -1.602828103253 & 2.64 & -2.429402029526 \\
\hline $\mathrm{C}$ & 1.294251182434 & 0.909565334216 & -2.129590197363 \\
\hline $\mathrm{H}$ & 1.566127052619 & -1.010438167608 & -1.294065056855 \\
\hline $\mathrm{H}$ & 0.819100642950 & & 35727 \\
\hline $\mathrm{H}$ & 2.344629169199 & 0.990846 & 31428 \\
\hline $\mathrm{H}$ & -3.443422119627 & 1.349809068380 & -1.582308944651 \\
\hline $\mathrm{H}$ & -1.165885878435 & -0.955697034267 & 2.879012135388 \\
\hline $\mathrm{N}$ & 0.141900099129 & 4.215730939567 & 0.342090955248 \\
\hline $\mathrm{H}$ & -0.270558036534 & 5.0660024914 & -0.010780652970 \\
\hline $\mathrm{H}$ & 1.064755678123 & 4.289460614092 & 0.737063476973 \\
\hline $\mathrm{H}$ & 1.250376414643 & -1.202182665149 & 2.911926827473 \\
\hline $\mathrm{H}$ & 1.879576016608 & 2.414809719090 & 0.989303534890 \\
\hline $\mathrm{C}$ & 3.095223735232 & 0.392468942226 & 2.036205324103 \\
\hline $\mathrm{C}$ & 3.554674593129 & -0.439833103881 & 0.862643596235 \\
\hline $\mathrm{H}$ & 3.333649483986 & -0.115313462975 & 2.971700655178 \\
\hline $\mathrm{H}$ & 3.606191343948 & 1.354643514126 & 2.033866436867 \\
\hline $\mathrm{C}$ & 3.415591889833 & -1.826629889400 & 0.880409713773 \\
\hline $\mathrm{C}$ & 4.092508627157 & 0.170141729594 & -0.266661280052 \\
\hline $\mathrm{C}$ & 3.783643932480 & -2.587270868045 & -0.221680232260 \\
\hline $\mathrm{H}$ & 3.017078317075 & -2.313581912862 & 1.763818622918 \\
\hline $\mathrm{C}$ & 4.471115083023 & -0.588418967965 & -1.367854451977 \\
\hline $\mathrm{H}$ & 4.213685020648 & 1.247979973475 & -0.283052214385 \\
\hline $\mathrm{C}$ & 4.309997425554 & -1.968227137856 & -1.350358550384 \\
\hline $\mathrm{H}$ & 3.666340891799 & -3.663755462926 & -0.197990963768 \\
\hline $\mathrm{H}$ & 4.889835687573 & -0.101670714479 & -2.240203391671 \\
\hline $\mathrm{H}$ & 4.602289527254 & -2.560571367561 & -2.208403185632 \\
\hline
\end{tabular}




\begin{tabular}{|c|c|c|c|}
\hline \multicolumn{4}{|c|}{ MA+_BNAH: } \\
\hline $\mathrm{H}$ & 1.746887679844 & 0.582673497540 & -1.392995894498 \\
\hline $\mathrm{C}$ & 0.951202708680 & 0.818658769123 & -2.111959104118 \\
\hline $\mathrm{C}$ & 0.295407205323 & -0.452077403809 & -2.581611239310 \\
\hline $\mathrm{H}$ & 1.465780627693 & 1.307403887748 & -2.950010749080 \\
\hline $\mathrm{C}$ & -0.078449588607 & 1.743699607795 & -1.499891846030 \\
\hline $\mathrm{C}$ & -1.021335808145 & -0.648764518118 & -2.536167988303 \\
\hline $\mathrm{C}$ & -1.392605807751 & 1.442657603847 & -1.500096676565 \\
\hline $\mathrm{C}$ & 0.316822131539 & 3.027910185210 & -0.892239432598 \\
\hline $\mathrm{N}$ & -1.909026938944 & 0.302981289397 & -2.037094441911 \\
\hline $\mathrm{O}$ & -0.482126005188 & 3.835329861603 & -0.419073684356 \\
\hline $\mathrm{H}$ & 1.697559601709 & -3.804808151797 & -0.479027042125 \\
\hline $\mathrm{C}$ & 2.478007397535 & -3.078837742467 & -0.287862685884 \\
\hline $\mathrm{C}$ & 2.101386259661 & -1.836086286098 & 0.289469423916 \\
\hline $\mathrm{C}$ & 3.781108296098 & -3.336597493071 & -0.577846991978 \\
\hline $\mathrm{C}$ & 3.089510415805 & -0.846165737381 & 0.546364327722 \\
\hline $\mathrm{C}$ & 0.777864272384 & -1.562404778545 & 0.610610328548 \\
\hline $\mathrm{C}$ & 4.761953100942 & -2.358173359641 & -0.291667521832 \\
\hline $\mathrm{H}$ & 4.076126346186 & -4.281115970968 & -1.014240737465 \\
\hline $\mathrm{C}$ & 4.440666533662 & -1.144611391400 & 0.250404840394 \\
\hline $\mathrm{N}$ & 2.718675132323 & 0.362833719246 & 1.065899537691 \\
\hline $\mathrm{C}$ & 0.425572561510 & -0.362404317937 & 1.207393414172 \\
\hline $\mathrm{H}$ & 5.801344232085 & -2.575051396291 & -0.503155908580 \\
\hline $\mathrm{H}$ & 5.226800523646 & -0.438911500694 & 0.465188332116 \\
\hline $\mathrm{C}$ & 1.432624957622 & 0.619125963921 & 1.447956634920 \\
\hline $\mathrm{C}$ & 3.752946677340 & 1.400116860264 & 1.194003544435 \\
\hline $\mathrm{C}$ & -0.924763710747 & -0.100444558271 & 1.570978592484 \\
\hline $\mathrm{C}$ & 1.053735565314 & 1.825234409999 & 2.085075657919 \\
\hline $\mathrm{H}$ & 3.291660975874 & 2.373448036591 & 1.277629099740 \\
\hline $\mathrm{H}$ & 4.372814119690 & 1.200341280433 & 2.067305017917 \\
\hline $\mathrm{H}$ & 4.361599450743 & 1.397916160473 & 0.294894062944 \\
\hline $\mathrm{C}$ & -1.263209596122 & 1.077408848410 & 2.156478316571 \\
\hline $\mathrm{H}$ & -1.660348228859 & -0.869748113964 & 1.368526203279 \\
\hline $\mathrm{C}$ & -0.254280200427 & 2.034880229563 & 2.418313617258 \\
\hline $\mathrm{H}$ & 1.778599144447 & 2.583023501252 & 2.332435983544 \\
\hline $\mathrm{H}$ & -2.288363862798 & 1.285177139416 & 2.432560593104 \\
\hline $\mathrm{H}$ & -0.522538808722 & 2.964215054185 & 2.904480962058 \\
\hline $\mathrm{H}$ & 0.011652749007 & -2.303431247662 & 0.407823068778 \\
\hline $\mathrm{H}$ & 0.919047993989 & -1.234514949966 & -2.994858445530 \\
\hline $\mathrm{N}$ & 1.649427917145 & 3.277451448002 & -0.880042305519 \\
\hline $\mathrm{H}$ & 1.975391011157 & 4.146108002830 & -0.485430497969 \\
\hline $\mathrm{H}$ & 2.326021764391 & 2.652162343853 & -1.285300955761 \\
\hline $\mathrm{H}$ & -1.485469566625 & -1.557392606384 & -2.896643394516 \\
\hline $\mathrm{H}$ & -2.108194669271 & 2.127759513649 & -1.061060183006 \\
\hline $\mathrm{C}$ & -3.328307393632 & -0.012211885995 & -1.912134662579 \\
\hline $\mathrm{C}$ & -3.661273560048 & -0.712858518751 & -0.616260387634 \\
\hline $\mathrm{H}$ & -3.600584305795 & -0.639101719951 & -2.761772340849 \\
\hline $\mathrm{H}$ & -3.886096171148 & 0.920859597997 & -1.985899894197 \\
\hline $\mathrm{C}$ & -4.191139864747 & -0.000022872961 & 0.454882376788 \\
\hline $\mathrm{C}$ & -3.400273196399 & -2.074080984068 & -0.461685617627 \\
\hline $\mathrm{C}$ & -4.449866657219 & -0.632047346204 & 1.665915477317 \\
\hline $\mathrm{H}$ & -4.400868904475 & 1.057848152868 & 0.339210622647 \\
\hline $\mathrm{C}$ & -3.653780062957 & -2.707315962701 & 0.747608590091 \\
\hline $\mathrm{H}$ & -2.999800448305 & -2.640273602012 & -1.295614779278 \\
\hline $\mathrm{C}$ & -4.178146341307 & -1.985871775285 & 1.815434574119 \\
\hline $\mathrm{H}$ & -4.862726754101 & -0.066444320765 & 2.492205937716 \\
\hline $\mathrm{H}$ & -3.448225576887 & -3.765032453758 & 0.856800636757 \\
\hline $\mathrm{H}$ & -4.378587484115 & -2.479813668301 & 2.757967344154 \\
\hline & & & \\
\hline $\mathrm{H}$ & -1.771000690644 & 0.385460695767 & 1.449086458535 \\
\hline
\end{tabular}




\begin{tabular}{|c|c|c|c|}
\hline $\mathrm{C}$ & -0.997408616036 & 0.556403484986 & 2.205088316294 \\
\hline $\mathrm{C}$ & -0.304250975909 & -0.730770083504 & 2.557864972856 \\
\hline $\mathrm{H}$ & -1.543132360084 & 0.946756941953 & 3.072549308860 \\
\hline $\mathrm{C}$ & 0.002309542664 & 1.578272740863 & 1.710900595192 \\
\hline $\mathrm{C}$ & 1.018579979798 & -0.877506438791 & 2.510837240013 \\
\hline $\mathrm{C}$ & 1.326425694486 & 1.319838191455 & 1.682645415117 \\
\hline $\mathrm{C}$ & -0.558502789511 & 2.852700592454 & 1.234889921623 \\
\hline $\mathrm{N}$ & 1.875711762990 & 0.148350447932 & 2.112548714898 \\
\hline $\mathrm{O}$ & -1.774539913093 & 2.973888405848 & 1.080746738273 \\
\hline $\mathrm{H}$ & -1.644857362446 & -3.849341334860 & 0.075105250314 \\
\hline $\mathrm{C}$ & -2.432913417826 & -3.118887116503 & -0.059514317946 \\
\hline $\mathrm{C}$ & -2.062282176482 & -1.815291173798 & -0.489798319988 \\
\hline $\mathrm{C}$ & -3.738052393777 & -3.427076889901 & 0.160133055668 \\
\hline $\mathrm{C}$ & -3.060623800557 & -0.814349862431 & -0.662996961826 \\
\hline $\mathrm{C}$ & -0.735875526107 & -1.498352242555 & -0.752624271018 \\
\hline $\mathrm{C}$ & -4.728644609602 & -2.439712721265 & -0.055810202012 \\
\hline $\mathrm{H}$ & -4.028230088584 & -4.417637295909 & 0.482883074205 \\
\hline $\mathrm{C}$ & -4.414954560600 & -1.169539040502 & -0.450744999465 \\
\hline $\mathrm{N}$ & -2.688969289827 & 0.447546557253 & -1.030483128251 \\
\hline $\mathrm{C}$ & -0.386633975526 & -0.241022632452 & -1.221223949245 \\
\hline $\mathrm{H}$ & -5.770268631541 & -2.696728822935 & 0.089321862485 \\
\hline $\mathrm{H}$ & -5.211792422807 & -0.464577085159 & -0.624266707262 \\
\hline $\mathrm{C}$ & -1.401458408567 & 0.747303652225 & -1.368765852271 \\
\hline $\mathrm{C}$ & -3.688881312773 & 1.526154121132 & -1.045368417348 \\
\hline $\mathrm{C}$ & 0.962156345776 & 0.074255066670 & -1.545624381945 \\
\hline $\mathrm{C}$ & -1.039900152136 & 2.018359000564 & -1.876619989089 \\
\hline $\mathrm{H}$ & -3.211025016987 & 2.441157967597 & -0.715015519994 \\
\hline $\mathrm{H}$ & -4.105086967881 & 1.637434164153 & -2.046115726592 \\
\hline $\mathrm{H}$ & -4.472435413358 & 1.297260801425 & -0.334723391208 \\
\hline $\mathrm{C}$ & 1.285896442917 & 1.309498224160 & -2.009861118945 \\
\hline $\mathrm{H}$ & 1.708546537291 & -0.700483217157 & -1.416739700243 \\
\hline $\mathrm{C}$ & 0.265889097123 & 2.276849529679 & -2.182662865218 \\
\hline $\mathrm{H}$ & -1.781090850239 & 2.782874729258 & -2.044802603053 \\
\hline $\mathrm{H}$ & 2.309398123211 & 1.556194857195 & -2.258959767491 \\
\hline $\mathrm{H}$ & 0.524905358163 & 3.251866565838 & -2.576032869661 \\
\hline $\mathrm{H}$ & 0.033629506497 & -2.250190321335 & -0.611763121901 \\
\hline $\mathrm{H}$ & -0.903366207560 & -1.571826917618 & 2.883491883176 \\
\hline $\mathrm{N}$ & 0.279310667022 & 3.882576125966 & 0.948111806352 \\
\hline $\mathrm{H}$ & -0.145800313357 & 4.762688679264 & 0.699365231314 \\
\hline $\mathrm{H}$ & 1.228741818648 & 3.908788119208 & 1.280632665908 \\
\hline $\mathrm{H}$ & 1.513829929035 & -1.799166548289 & 2.786778755697 \\
\hline $\mathrm{H}$ & 2.048327522832 & 2.032495260186 & 1.300760002185 \\
\hline $\mathrm{C}$ & 3.305768600264 & -0.106222164106 & 1.972091177296 \\
\hline $\mathrm{C}$ & 3.674955413604 & -0.675566688983 & 0.622771493696 \\
\hline $\mathrm{H}$ & 3.593226188788 & -0.797286116221 & 2.765054706138 \\
\hline $\mathrm{H}$ & 3.831265517204 & 0.834227670910 & 2.135884271809 \\
\hline $\mathrm{C}$ & 4.206403646346 & 0.144756373851 & -0.367498027787 \\
\hline $\mathrm{C}$ & 3.450750418627 & -2.022474568916 & 0.338779113481 \\
\hline $\mathrm{C}$ & 4.502560421022 & -0.366772823761 & -1.625959625743 \\
\hline $\mathrm{H}$ & 4.388793457621 & 1.191839388512 & -0.151030460201 \\
\hline $\mathrm{C}$ & 3.741602603077 & -2.535472081960 & -0.918021478334 \\
\hline $\mathrm{H}$ & 3.050096224504 & -2.672269611910 & 1.109144917641 \\
\hline $\mathrm{C}$ & 4.267157639444 & -1.706846289157 & -1.904415256432 \\
\hline $\mathrm{H}$ & 4.916565197937 & 0.281744850516 & -2.388277164146 \\
\hline $\mathrm{H}$ & 3.564529014557 & -3.583115633301 & -1.127735613325 \\
\hline $\mathrm{H}$ & 4.496998771238 & -2.107112560117 & -2.883932726899 \\
\hline \multicolumn{4}{|c|}{$\mathrm{MA}+\mathrm{BNAH}: \quad \mathrm{G} 38$} \\
\hline $\mathrm{H}$ & 0.723650087413 & 1.470599079518 & -1.834088931924 \\
\hline $\mathrm{C}$ & -0.247651747363 & 1.730343868752 & -1.394412010191 \\
\hline $\mathrm{C}$ & -1.368130966381 & 1.280528386837 & -2.290616250588 \\
\hline $\mathrm{H}$ & -0.233298577957 & 2.823129216474 & -1.314376855085 \\
\hline
\end{tabular}




\begin{tabular}{|c|c|c|c|}
\hline $\mathrm{C}$ & -0.384390405752 & 1.103014420953 & -0.026353038918 \\
\hline $\mathrm{C}$ & -2.304191589631 & 0.414486767923 & -1.905388455078 \\
\hline $\mathrm{C}$ & -1.372602403872 & 0.230570472166 & 0.262456561849 \\
\hline $\mathrm{C}$ & 0.679412632144 & 1.411893376036 & 0.941154845907 \\
\hline $\mathrm{N}$ & -2.322097156478 & -0.155234198387 & -0.633109442729 \\
\hline $\mathrm{O}$ & 1.704136313295 & 1.983872281717 & 0.574209212398 \\
\hline $\mathrm{H}$ & 0.818486113403 & -1.171783451419 & -2.944783391875 \\
\hline $\mathrm{C}$ & 0.845258266912 & -1.500553906838 & -1.912980853882 \\
\hline $\mathrm{C}$ & 1.910099334862 & -1.028582214565 & -1.094768434785 \\
\hline $\mathrm{C}$ & -0.091072814942 & -2.345094622840 & -1.408607044152 \\
\hline $\mathrm{C}$ & 1.975933011498 & -1.409490390352 & 0.277000748160 \\
\hline $\mathrm{C}$ & 2.894465245222 & -0.202721491835 & -1.615455051437 \\
\hline $\mathrm{C}$ & 0.004745777604 & -2.754469414579 & -0.057204634782 \\
\hline $\mathrm{H}$ & -0.901305264565 & -2.710030829538 & -2.025788051028 \\
\hline $\mathrm{C}$ & 0.994231908711 & -2.304986277910 & 0.771522684300 \\
\hline $\mathrm{N}$ & 2.978870713970 & -0.915048773170 & 1.058810395591 \\
\hline $\mathrm{C}$ & 3.957199036454 & 0.220337096740 & -0.829359892990 \\
\hline $\mathrm{H}$ & -0.726523224908 & -3.449058885367 & 0.337302803010 \\
\hline $\mathrm{H}$ & 1.027336144901 & -2.671444489828 & 1.784455529318 \\
\hline $\mathrm{C}$ & 3.991388990254 & -0.159513930680 & 0.539654650621 \\
\hline $\mathrm{C}$ & 2.99476 & -1.184966990252 & 2.503 \\
\hline $\mathrm{C}$ & 4.99463 & 1.029587426607 & 20909 \\
\hline $\mathrm{C}$ & 5.090644938684 & 0.252506431970 & 1.329914832313 \\
\hline $\mathrm{H}$ & 3.272060232793 & -0.274399874745 & 3.025387640433 \\
\hline $\mathrm{H}$ & 2.006121767346 & -1.463284893103 & 2.837715085437 \\
\hline $\mathrm{H}$ & 3.707 & 43232 & 2.725 \\
\hline $\mathrm{C}$ & 6.031831799897 & 1.428579623807 & -0.581394793711 \\
\hline $\mathrm{H}$ & 4.930550257025 & 1.309712237849 & -2.409042825345 \\
\hline $\mathrm{C}$ & 6.074448748522 & 1.021174376936 & 0.772569574419 \\
\hline $\mathrm{H}$ & 5.180422668650 & -0.049122275329 & 7400 \\
\hline $\mathrm{H}$ & 6.826 & 2323 & 46111308 \\
\hline $\mathrm{H}$ & 6.9135 & 10086 & 1.387829170288 \\
\hline $\mathrm{H}$ & 2.844692382859 & 0.097463765706 & -2.656620792438 \\
\hline $\mathrm{H}$ & -1.412964967737 & 1.667846274733 & -3.300424565181 \\
\hline $\mathrm{N}$ & 0.5381 & & 241521 \\
\hline $\mathrm{H}$ & -0.3708 & 0.820544864326 & 2.627183012512 \\
\hline $\mathrm{H}$ & 1.240162504333 & 1.341979390225 & 2.882288164051 \\
\hline $\mathrm{H}$ & -1.456380639515 & -0.250251625226 & 1.229294289168 \\
\hline $\mathrm{H}$ & -3.105172912477 & 0.090030514437 & -2.557461085790 \\
\hline $\mathrm{C}$ & -3.424560575982 & 2782 & -0.247599041585 \\
\hline $\mathrm{C}$ & -4.7070 & -0.266739435572 & 182823901 \\
\hline $\mathrm{H}$ & -3.111538918205 & -1.584943526703 & 0.627371863953 \\
\hline $\mathrm{H}$ & -3.609448226154 & -1.728819965058 & -1.050566477309 \\
\hline $\mathrm{C}$ & -4.722144636610 & 1.102929488840 & 0.302491571354 \\
\hline $\mathrm{C}$ & -5.903131414506 & -0.979314037894 & 0.130880982859 \\
\hline $\mathrm{C}$ & -5.914698039004 & 1.750303075310 & 0.610193238667 \\
\hline $\mathrm{H}$ & -3.802257005845 & 1.673166403431 & 0.245039674924 \\
\hline $\mathrm{C}$ & -7.092109217209 & -0.335784665929 & 0.440673127569 \\
\hline $\mathrm{H}$ & -5.899254195237 & -2.046331405952 & -0.063855556585 \\
\hline $\mathrm{C}$ & -7.101148498982 & 1.034318381963 & 0.681693157835 \\
\hline $\mathrm{H}$ & -5.913330066785 & 2.817988556529 & 0.791720731753 \\
\hline $\mathrm{H}$ & -8.014163159666 & -0.901804744279 & 0.489091954449 \\
\hline $\mathrm{H}$ & -8.029058364029 & 1.539408447983 & 0.918841131547 \\
\hline \multicolumn{4}{|c|}{$\mathrm{MA}+$ BNAH: $\quad$ G40 } \\
\hline $\mathrm{H}$ & -1.808138475680 & 1.211947736311 & 0.965078567468 \\
\hline $\mathrm{C}$ & -0.953260847335 & 1.515534087129 & 1.574168904042 \\
\hline $\mathrm{C}$ & -0.424012307916 & 0.357990535819 & 2.377164798056 \\
\hline $\mathrm{H}$ & -1.349720030505 & 2.294017893515 & 2.239353127915 \\
\hline $\mathrm{C}$ & 0.136890009814 & 2.081409932916 & 0.691529462560 \\
\hline $\mathrm{C}$ & 0.877888044290 & 0.091148069088 & 2.477695687691 \\
\hline $\mathrm{C}$ & 1.428614916278 & 1.734467967345 & 0.865863118613 \\
\hline
\end{tabular}




\begin{tabular}{|c|c|c|c|}
\hline $\mathrm{C}$ & -0.290998604139 & 3.029379036651 & -0.352648029363 \\
\hline $\mathrm{N}$ & 1.845163006443 & 0.828589152945 & 1.797367511298 \\
\hline $\mathrm{O}$ & -1.486817583179 & 3.207814067737 & -0.578595134350 \\
\hline $\mathrm{H}$ & -5.128506028544 & 1.186653401983 & -1.462744048813 \\
\hline $\mathrm{C}$ & -4.857581936907 & 0.393878624703 & -0.776600460876 \\
\hline $\mathrm{C}$ & -3.520092595667 & -0.087108413706 & -0.814838288456 \\
\hline $\mathrm{C}$ & -5.752094998087 & -0.123713486051 & 0.105727370354 \\
\hline $\mathrm{C}$ & -3.116558956337 & -1.136323338630 & 0.061069443577 \\
\hline $\mathrm{C}$ & -2.586196294354 & 0.470930377269 & -1.677787587851 \\
\hline $\mathrm{C}$ & -5.335241851671 & -1.141453227151 & 0.995553384671 \\
\hline $\mathrm{H}$ & -6.769475532256 & 0.240972350592 & 0.144398329911 \\
\hline $\mathrm{C}$ & -4.063788584274 & -1.643154071794 & 0.983398782048 \\
\hline $\mathrm{N}$ & -1.833640775424 & -1.602280435002 & 0.000673775171 \\
\hline $\mathrm{C}$ & -1.268579787516 & 0.037596468003 & -1.674766363590 \\
\hline $\mathrm{H}$ & -6.040836664575 & -1.533040231777 & 1.717325696638 \\
\hline $\mathrm{H}$ & -3.796773525735 & -2.398968602858 & 1.703679591614 \\
\hline $\mathrm{C}$ & -0.891140633027 & -1.013394130120 & -0.793195152828 \\
\hline $\mathrm{C}$ & -1.434186584809 & -2.772563751104 & 0.794667459465 \\
\hline $\mathrm{C}$ & -0.294968600897 & 0.637027326886 & -2.519659880627 \\
\hline $\mathrm{C}$ & 0.464930276697 & -1.416196654037 & -0.759716428706 \\
\hline $\mathrm{H}$ & -0.779454035972 & -3.394088454068 & 0.192909341289 \\
\hline $\mathrm{H}$ & -0.919517503173 & -2.451341802395 & 1.700111651780 \\
\hline $\mathrm{H}$ & -2.305837892440 & -3.360949725279 & 1.044873960125 \\
\hline $\mathrm{C}$ & 0.997673857192 & 0.218547979100 & -2.484177307707 \\
\hline $\mathrm{H}$ & -0.617543801275 & 1.437346036167 & -3.174085925882 \\
\hline $\mathrm{C}$ & 1.369582103463 & -0.810570573952 & -1.586825610414 \\
\hline $\mathrm{H}$ & 0.805599549810 & -2.176841518905 & -0.074533663700 \\
\hline $\mathrm{H}$ & 1.746203397709 & 0.672505407927 & -3.119964280490 \\
\hline $\mathrm{H}$ & 2.403996185654 & -1.127362403106 & -1.546809198744 \\
\hline $\mathrm{H}$ & -2.885892361712 & 1.268107537976 & -2.348154315864 \\
\hline $\mathrm{H}$ & -1.123268643519 & -0.266359367690 & 2.919386762227 \\
\hline $\mathrm{N}$ & 0.652590135750 & 3.685866088460 & -1.079231191595 \\
\hline $\mathrm{H}$ & 0.327027854890 & 4.399392909890 & -1.713096145333 \\
\hline $\mathrm{H}$ & 1.614554454219 & 3.735796800284 & -0.788913214757 \\
\hline $\mathrm{H}$ & 1.265806802978 & -0.717591794278 & 3.083598871247 \\
\hline $\mathrm{H}$ & 2.229597595312 & 2.141415381343 & 0.258926273580 \\
\hline $\mathrm{C}$ & 3.264392953392 & 0.524306074935 & 1.950688670659 \\
\hline $\mathrm{C}$ & 3.802621239375 & -0.430585679123 & 0.911478059170 \\
\hline $\mathrm{H}$ & 3.397201584036 & 375110536 & 2.947885936603 \\
\hline $\mathrm{H}$ & 3.817487566172 & 1.462785134492 & 1.907499572726 \\
\hline $\mathrm{C}$ & 4.575862222696 & 0.039101990503 & -0.145990924550 \\
\hline $\mathrm{C}$ & 3.528689640079 & -1.794966723555 & 0.996700575139 \\
\hline $\mathrm{C}$ & 5.071020719250 & -0.838574971508 & -1.104739400228 \\
\hline $\mathrm{H}$ & 4.799719393157 & 1.098108663826 & -0.212732305989 \\
\hline $\mathrm{C}$ & 4.017350447520 & -2.673210446381 & 0.039593755421 \\
\hline $\mathrm{H}$ & 2.935100001769 & -2.169404847434 & 1.824036062764 \\
\hline $\mathrm{C}$ & 4.792343288718 & -2.195828366109 & -1.013329002970 \\
\hline $\mathrm{H}$ & 5.675305042476 & -0.461889459359 & -1.920686237995 \\
\hline $\mathrm{H}$ & 3.801179907562 & -3.731670051671 & 0.116913269952 \\
\hline $\mathrm{H}$ & 5.178452885767 & -2.881292403951 & $-1.75733289363 ?$ \\
\hline \multicolumn{4}{|c|}{$\mathrm{MA}+$ BNAH: $\quad$ G43a } \\
\hline $\mathrm{H}$ & 0.929404212102 & 2.126430527367 & -1.018889634440 \\
\hline $\mathrm{C}$ & 0.212331756683 & 2.626849920964 & -0.35358638639 \\
\hline $\mathrm{C}$ & -1.061611558285 & 2.914069127174 & -1.099459646084 \\
\hline $\mathrm{H}$ & 0.697856331823 & 3.570171437912 & -0.069791229379 \\
\hline $\mathrm{C}$ & -0.077672021408 & 1.778314143038 & 0.865237806399 \\
\hline $\mathrm{C}$ & -2.260996550221 & 2.531524755974 & -0.663931262866 \\
\hline $\mathrm{C}$ & -1.336715981689 & 1.439068692261 & 1.215308528186 \\
\hline $\mathrm{C}$ & 1.012423744225 & 1.312105767054 & 1.738672230782 \\
\hline $\mathrm{N}$ & -2.440837778223 & 1.814032738569 & 0.517057980465 \\
\hline $\mathrm{O}$ & 0.844340177197 & 0.594494582325 & 2.726434397398 \\
\hline
\end{tabular}




\begin{tabular}{|c|c|c|c|}
\hline $\mathrm{H}$ & -0.513407441118 & -0.188719897490 & -2.572014132763 \\
\hline $\mathrm{C}$ & -0.333553106882 & -0.698761114438 & -1.633476704579 \\
\hline $\mathrm{C}$ & 1.000419975940 & -0.718802199030 & -1.133908817645 \\
\hline $\mathrm{C}$ & -1.331794056904 & -1.323211412686 & -0.955906975775 \\
\hline $\mathrm{C}$ & 1.284201291972 & -1.355708745338 & 0.108600110125 \\
\hline $\mathrm{C}$ & 2.038518283590 & -0.132918583054 & -1.842164130288 \\
\hline $\mathrm{C}$ & -1.029538488338 & -1.997849161366 & 0.253393082912 \\
\hline $\mathrm{H}$ & -2.347682313873 & -1.324676136225 & -1.331141809433 \\
\hline $\mathrm{C}$ & 0.227279287440 & -2.011904184127 & 0.785524064100 \\
\hline $\mathrm{N}$ & 2.555760716027 & -1.330959171679 & 0.600138135795 \\
\hline $\mathrm{C}$ & 3.344955221731 & -0.197079674961 & -1.373960359812 \\
\hline $\mathrm{H}$ & -1.818632200830 & -2.525834644887 & 0.772683969121 \\
\hline $\mathrm{H}$ & 0.402437274872 & -2.554536054859 & 1.700144499464 \\
\hline $\mathrm{C}$ & 3.597178518848 & -0.827294330590 & -0.124291378355 \\
\hline $\mathrm{C}$ & 2.824587334811 & -1.831545326969 & 1.956121019194 \\
\hline $\mathrm{C}$ & 4.422251536659 & 0.361841935843 & -2.113738811340 \\
\hline $\mathrm{C}$ & 4.933116208978 & -0.912107785483 & 0.335332893088 \\
\hline $\mathrm{H}$ & 3.559708122133 & -1.186241583907 & 2.425592242710 \\
\hline $\mathrm{H}$ & 1.920776687674 & -1.771729974415 & 2.545553273053 \\
\hline $\mathrm{H}$ & 3.194917176152 & -2.855271933595 & 1.911301694895 \\
\hline $\mathrm{C}$ & 5.695334715882 & 0.282688357252 & -1.643316819963 \\
\hline $\mathrm{H}$ & 4.193359695910 & 0.842934301194 & -3.056556423215 \\
\hline $\mathrm{C}$ & 5.941407526501 & -0.371722853827 & -0.413138637676 \\
\hline $\mathrm{H}$ & 5.174541479947 & -1.422448100375 & 1.254045183638 \\
\hline $\mathrm{H}$ & 6.520173619015 & 0.703530958805 & -2.202065575642 \\
\hline $\mathrm{H}$ & 6.959322673059 & -0.454878376157 & -0.053948101557 \\
\hline $\mathrm{H}$ & 1.830617157212 & 0.364367463444 & -2.783680171952 \\
\hline $\mathrm{H}$ & -1.005849903559 & 3.476631761072 & -2.022319173649 \\
\hline $\mathrm{N}$ & 2.256605518109 & 1.711785428129 & 1.377664124826 \\
\hline $\mathrm{H}$ & 2.422092527967 & 2.356425193108 & 0.622344222054 \\
\hline $\mathrm{H}$ & 3.034492520929 & 1.457410268015 & 1.967705856397 \\
\hline $\mathrm{H}$ & -1.514148144622 & 0.844777297250 & 2.103740081589 \\
\hline $\mathrm{H}$ & -3.172340520622 & 2.766469545073 & -1.197667172182 \\
\hline $\mathrm{C}$ & -3.784835207238 & 1.434842761227 & 0.943913849218 \\
\hline $\mathrm{C}$ & -4.287688521641 & 0.177130181355 & 0.275717876610 \\
\hline $\mathrm{H}$ & -4.449870978636 & 2.270222338322 & 0.720550633023 \\
\hline $\mathrm{H}$ & -3.761543672750 & 1.299780129961 & 2.024387512736 \\
\hline $\mathrm{C}$ & -4.803316891413 & 0.219133592690 & -1.019268738404 \\
\hline $\mathrm{C}$ & -4.213350772012 & -1.046555856427 & 0.933252658046 \\
\hline $\mathrm{C}$ & -5.214797917238 & -0.945591431518 & -1.652565969448 \\
\hline $\mathrm{H}$ & -4.888122056119 & 1.170900424990 & -1.531425934790 \\
\hline $\mathrm{C}$ & -4.630542880370 & -2.214072652648 & 0.304555347164 \\
\hline $\mathrm{H}$ & -3.823582182464 & -1.084968842203 & 1.944740254783 \\
\hline $\mathrm{C}$ & -5.125801035702 & -2.166545622190 & -0.992064410798 \\
\hline $\mathrm{H}$ & -5.611864594950 & -0.901245020161 & -2.659134412690 \\
\hline $\mathrm{H}$ & -4.567421150987 & -3.160498331496 & 0.827605416675 \\
\hline $\mathrm{H}$ & -5.450025125292 & -3.075012578266 & -1.484124153328 \\
\hline \multicolumn{4}{|c|}{ MA+ BNAH: $\quad$ G47a } \\
\hline $\mathrm{H}$ & -1.319679909178 & -1.825146562000 & -1.985363644435 \\
\hline $\mathrm{C}$ & -0.309928735195 & -1.966652248546 & -1.576242930462 \\
\hline $\mathrm{C}$ & 0.680156318823 & -1.154192056395 & -2.367675603212 \\
\hline $\mathrm{H}$ & -0.099866592829 & -3.037579104178 & -1.695662025467 \\
\hline $\mathrm{C}$ & -0.261678603581 & -1.565152440903 & -0.118367673666 \\
\hline $\mathrm{C}$ & 1.506817517842 & -0.269724913033 & -1.811969719325 \\
\hline $\mathrm{C}$ & 0.622264102591 & -0.659478087727 & 0.344514757681 \\
\hline $\mathrm{C}$ & -1.195035586853 & -2.146279117679 & 0.864279531104 \\
\hline $\mathrm{N}$ & 1.504806563057 & 0.012892512224 & -0.445815523870 \\
\hline $\mathrm{O}$ & -1.213080106032 & -1.828095838912 & 2.055328441940 \\
\hline $\mathrm{H}$ & -0.270995105242 & 2.445394685185 & -2.864986510542 \\
\hline $\mathrm{C}$ & -0.266738717823 & 2.568877847213 & -1.789032647709 \\
\hline $\mathrm{C}$ & -1.196889708564 & 1.806134782497 & -1.029574334568 \\
\hline
\end{tabular}




\begin{tabular}{|c|c|c|c|}
\hline $\mathrm{C}$ & 0.588050086921 & 3.425829353703 & -1.172545175119 \\
\hline $\mathrm{C}$ & -1.212140472885 & 1.916392710882 & 0.390116357986 \\
\hline $\mathrm{C}$ & -2.089843470876 & 0.943512089123 & -1.645834201770 \\
\hline $\mathrm{C}$ & 0.542588033283 & 3.556676793479 & 0.236984258833 \\
\hline $\mathrm{H}$ & 1.294486037127 & 4.013081583896 & -1.743562328138 \\
\hline $\mathrm{C}$ & -0.319135754173 & 2.827732263598 & 1.006614503803 \\
\hline $\mathrm{N}$ & -2.079102294128 & 1.152658666372 & 1.114257937407 \\
\hline $\mathrm{C}$ & -3.038768871586 & 0.252148229600 & -0.902695374541 \\
\hline $\mathrm{H}$ & 1.208870644872 & 4.258308089418 & 0.723115042785 \\
\hline $\mathrm{H}$ & -0.329428614526 & 2.983938573170 & 2.073177078823 \\
\hline $\mathrm{C}$ & -3.036028899207 & 0.385630409221 & 0.513777205396 \\
\hline $\mathrm{C}$ & -1.983184936938 & 1.150254084097 & 2.581481143006 \\
\hline $\mathrm{C}$ & -4.008463710166 & -0.575141788668 & -1.533715146888 \\
\hline $\mathrm{C}$ & -4.038209586620 & -0.281118741814 & 1.259126460543 \\
\hline $\mathrm{H}$ & -2.625592565445 & 1.924624513466 & 3.000079656443 \\
\hline $\mathrm{H}$ & -2.261335007598 & 0.169813713777 & 2.945485496087 \\
\hline $\mathrm{H}$ & -0.952679981148 & 1.319463127640 & 2.870136127570 \\
\hline $\mathrm{C}$ & -4.955510127486 & -1.211496713121 & -0.794772820008 \\
\hline $\mathrm{H}$ & -3.972512801093 & -0.664127338038 & -2.612574573677 \\
\hline $\mathrm{C}$ & -4.967395159626 & -1.044981998388 & 0.610900589638 \\
\hline $\mathrm{H}$ & -4.099251682470 & -0.172592815870 & 2.330109361742 \\
\hline $\mathrm{H}$ & -5.702858195919 & -1.833731984291 & -1.268228985280 \\
\hline $\mathrm{H}$ & -5.737421141567 & -1.534781573785 & 1.193538062169 \\
\hline $\mathrm{H}$ & -2.066716362386 & 0.829001032371 & -2.724579894154 \\
\hline $\mathrm{H}$ & 0.726845286274 & -1.297631130715 & -3.439471744432 \\
\hline $\mathrm{N}$ & -2.058904929294 & -3.063547029533 & 0.375226514246 \\
\hline $\mathrm{H}$ & -2.742935799484 & -3.464933158074 & 0.997969849754 \\
\hline $\mathrm{H}$ & -2.077749799048 & -3.331454613207 & -0.594797442757 \\
\hline $\mathrm{H}$ & 2.222053553826 & 0.302496890901 & -2.389637903567 \\
\hline $\mathrm{H}$ & 0.647537776352 & -0.411302246916 & 1.399758492908 \\
\hline $\mathrm{C}$ & 2.594143092521 & 0.775046563428 & 0.128672696212 \\
\hline $\mathrm{C}$ & 3.877580669888 & -0.011261036305 & 0.305424935072 \\
\hline $\mathrm{H}$ & 2.260377193254 & 1.156829848962 & 1.095684797157 \\
\hline $\mathrm{H}$ & 2.784721302052 & 1.641804121877 & -0.508298799851 \\
\hline $\mathrm{C}$ & 5.051401927702 & 0.677148484204 & 0.609595887546 \\
\hline $\mathrm{C}$ & 3.917706441907 & -1.396473973753 & 0.192593497372 \\
\hline $\mathrm{C}$ & 6.241758971882 & -0.006982377815 & 0.804302337414 \\
\hline $\mathrm{H}$ & 5.027972931600 & 1.758441364019 & 0.692751241659 \\
\hline $\mathrm{C}$ & 5.112326168652 & -2.084110523291 & 0.385143196821 \\
\hline $\mathrm{H}$ & 3.016892755930 & -1.948487964963 & -0.049008115252 \\
\hline $\mathrm{C}$ & 6.275609603677 & -1.393471164688 & 0.692278948864 \\
\hline $\mathrm{H}$ & 7.145763962104 & 0.540847616209 & 1.040315265314 \\
\hline $\mathrm{H}$ & 5.129921475046 & -3.163019638444 & 0.292531821233 \\
\hline $\mathrm{H}$ & 7.204799811781 & -1.929070769479 & 0.840815624163 \\
\hline & JAH: $\quad$ G4a & & \\
\hline $\mathrm{H}$ & -1.720973143995 & 0.206798582993 & -2.099009446590 \\
\hline $\mathrm{C}$ & -0.982163027686 & -0.522636294729 & -2.449927470746 \\
\hline $\mathrm{C}$ & 0.299662225718 & 0.182746965476 & -2.808555706234 \\
\hline $\mathrm{H}$ & -1.429310042929 & -0.977407160237 & -3.345071336649 \\
\hline $\mathrm{C}$ & -0.713806086530 & -1.566464258220 & -1.387485072928 \\
\hline $\mathrm{C}$ & 1.496129933793 & -0.253347338671 & -2.416291860156 \\
\hline $\mathrm{C}$ & 0.542046730475 & -1.936428965782 & -1.062109914982 \\
\hline $\mathrm{C}$ & -1.817707455909 & -2.256456280132 & -0.696724145937 \\
\hline $\mathrm{N}$ & 1.657115024745 & -1.376328361154 & -1.605501966094 \\
\hline $\mathrm{O}$ & -1.649341137881 & -3.038928775772 & 0.241415015958 \\
\hline $\mathrm{H}$ & -0.857610608943 & 4.017983670009 & -1.704574599091 \\
\hline $\mathrm{C}$ & -0.270437902610 & 3.367249069575 & -1.068530096412 \\
\hline $\mathrm{C}$ & -0.956955847223 & 2.359961349172 & -0.335966978176 \\
\hline $\mathrm{C}$ & 1.076020932056 & 3.506403986040 & -0.951723015406 \\
\hline $\mathrm{C}$ & -0.226668578576 & 1.465291583024 & 0.499348455681 \\
\hline $\mathrm{C}$ & -2.336839704006 & 2.234114580932 & -0.417175872177 \\
\hline
\end{tabular}




\begin{tabular}{|c|c|c|c|}
\hline $\mathrm{C}$ & 1.789406759025 & 2.639438153346 & -0.090801331659 \\
\hline $\mathrm{H}$ & 1.604463789054 & 4.275650905775 & -1.498287020450 \\
\hline $\mathrm{C}$ & 1.175253484325 & 1.641140748040 & 0.612700177142 \\
\hline $\mathrm{N}$ & -0.896359189350 & 0.486519130152 & 1.174814037963 \\
\hline $\mathrm{C}$ & -3.009868766538 & 1.280069878359 & 0.332205244237 \\
\hline $\mathrm{H}$ & 2.858413210517 & 2.769687765013 & 0.021083240896 \\
\hline $\mathrm{H}$ & 1.772452410832 & 1.026903187247 & 1.270699280022 \\
\hline $\mathrm{C}$ & -2.257745875209 & 0.402837956938 & 1.163365858814 \\
\hline $\mathrm{C}$ & -0.141555254171 & -0.534448410335 & 1.914834185526 \\
\hline $\mathrm{C}$ & -4.426971464749 & 1.165223209069 & 0.283125874735 \\
\hline $\mathrm{C}$ & -2.952313028102 & -0.532292942612 & 1.968855579946 \\
\hline $\mathrm{H}$ & -0.632013944973 & -1.494265574622 & 1.782122694623 \\
\hline $\mathrm{H}$ & 0.851992776890 & -0.615601587914 & 1.495931166837 \\
\hline $\mathrm{H}$ & -0.085050888504 & -0.267844148418 & 2.970190247061 \\
\hline $\mathrm{C}$ & -5.068159253433 & 0.242441546278 & 1.046825208701 \\
\hline $\mathrm{H}$ & -4.967414331003 & 1.838574993690 & -0.370414465706 \\
\hline $\mathrm{C}$ & -4.314815568668 & -0.596419749372 & 1.904634919641 \\
\hline $\mathrm{H}$ & -2.424724034775 & -1.182025710865 & 2.648402583144 \\
\hline $\mathrm{H}$ & -6.145494021804 & 0.150972709914 & 1.018206646008 \\
\hline $\mathrm{H}$ & -4.833212792328 & -1.309669555577 & 2.532985257768 \\
\hline $\mathrm{H}$ & -2.896909560599 & 2.899383035613 & -1.065474053384 \\
\hline $\mathrm{H}$ & 0.251435557070 & 1.065133413116 & -3.433507392386 \\
\hline $\mathrm{N}$ & -3.060909020528 & -1.969607853046 & -1.148586049203 \\
\hline $\mathrm{H}$ & -3.849546146574 & -2.425681164903 & -0.716252156671 \\
\hline $\mathrm{H}$ & -3.225490898358 & -1.392674964368 & -1.956658135990 \\
\hline $\mathrm{H}$ & 2.417296819379 & 0.241616763876 & -2.696629294505 \\
\hline $\mathrm{H}$ & 0.709990839401 & -2.720214101951 & -0.333135525312 \\
\hline $\mathrm{C}$ & 2.992835808741 & -1.810647882860 & -1.205544940357 \\
\hline $\mathrm{C}$ & 3.517988362989 & -1.074929765336 & 0.003690405177 \\
\hline $\mathrm{H}$ & 3.657631443488 & -1.658424843839 & -2.055931147087 \\
\hline $\mathrm{H}$ & 2.943373682211 & -2.879760119785 & -1.001572860942 \\
\hline $\mathrm{C}$ & 4.250942369180 & 0.099926098347 & -0.142844162427 \\
\hline $\mathrm{C}$ & 3.242357917091 & -1.545033016892 & 1.285911274897 \\
\hline $\mathrm{C}$ & 4.687230524772 & 0.803381356350 & 0.972975858217 \\
\hline $\mathrm{H}$ & 4.482024169353 & 0.463943531782 & -1.138025896311 \\
\hline $\mathrm{C}$ & 3.670011251334 & -0.840356794316 & 2.403845299355 \\
\hline $\mathrm{H}$ & 2.685314057073 & -2.468057908378 & 1.407340785114 \\
\hline $\mathrm{C}$ & 4.391382769399 & 0.338213964107 & 2.248633707490 \\
\hline $\mathrm{H}$ & 5.258196213197 & 1.715140993503 & 0.846964199360 \\
\hline $\mathrm{H}$ & 3.446292359780 & -1.213518224330 & 3.395719422266 \\
\hline $\mathrm{H}$ & 4.729648174067 & 0.886407646679 & 3.119048827387 \\
\hline \multicolumn{4}{|c|}{$\mathrm{MA}+$ BNAH: $\quad$ G4 } \\
\hline $\mathrm{H}$ & 0.557371165147 & & -1.168175090535 \\
\hline $\mathrm{C}$ & 0.708987153328 & 1.674225389845 & -0.374728158629 \\
\hline $\mathrm{C}$ & -0.608522579898 & 2.216326991153 & 0.108652193682 \\
\hline $\mathrm{H}$ & 1.305423568461 & 2.468182322811 & -0.841550681607 \\
\hline $\mathrm{C}$ & 1.470521589770 & 1.045641075701 & 0.770662389599 \\
\hline $\mathrm{C}$ & -0.913334013651 & 2.316556092522 & 1.401177426943 \\
\hline $\mathrm{C}$ & 1.073154235901 & 1.203664729520 & 2.050703818913 \\
\hline $\mathrm{C}$ & 2.677330016160 & 0.288577777237 & 0.396880131164 \\
\hline $\mathrm{N}$ & -0.048334294728 & 1.891602193745 & 2.410501398153 \\
\hline $\mathrm{O}$ & 3.016030813460 & 0.212050313420 & -0.782059437573 \\
\hline $\mathrm{H}$ & -3.154132692428 & 0.109094684249 & -2.483086651111 \\
\hline $\mathrm{C}$ & -2.940742595969 & -0.171042479072 & -1.459010931077 \\
\hline $\mathrm{C}$ & -1.710583983208 & -0.832999556985 & -1.194045669782 \\
\hline $\mathrm{C}$ & -3.818306298021 & 0.080200616984 & -0.451958689404 \\
\hline $\mathrm{C}$ & -1.377020262515 & -1.200574034127 & 0.140653892131 \\
\hline $\mathrm{C}$ & -0.823227812126 & -1.136018478295 & -2.218358123123 \\
\hline $\mathrm{C}$ & -3.497604044193 & -0.325636299476 & 0.864838750732 \\
\hline $\mathrm{H}$ & -4.760097633234 & 0.574739786799 & -0.647361632260 \\
\hline $\mathrm{C}$ & -2.315153295635 & -0.939896004484 & 1.170174157828 \\
\hline
\end{tabular}




\begin{tabular}{|c|c|c|c|}
\hline $\mathrm{N}$ & -0.178910372370 & -1.806078930058 & 0.388013447689 \\
\hline $\mathrm{C}$ & 0.353104587025 & -1.824445920942 & -1.960830079979 \\
\hline $\mathrm{H}$ & -4.210523922786 & -0.147619011133 & 1.660169250955 \\
\hline $\mathrm{H}$ & -2.132750202822 & -1.243756931060 & 2.190876790232 \\
\hline $\mathrm{C}$ & 0.663110726752 & -2.181624868354 & -0.617537269091 \\
\hline $\mathrm{C}$ & 0.220766131798 & -2.049572209547 & 1.780275156452 \\
\hline $\mathrm{C}$ & 1.245644600524 & -2.178971930826 & -3.010040714949 \\
\hline $\mathrm{C}$ & 1.833564891940 & -2.938930775067 & -0.369767405425 \\
\hline $\mathrm{H}$ & 1.298541501095 & -1.964437329449 & 1.861659204700 \\
\hline $\mathrm{H}$ & -0.214660223439 & -1.283019384082 & 2.408026853300 \\
\hline $\mathrm{H}$ & -0.114076248097 & -3.035894430079 & 2.101885687495 \\
\hline $\mathrm{C}$ & 2.375315610632 & -2.883900065081 & -2.742499944379 \\
\hline $\mathrm{H}$ & 0.987837208743 & -1.877442887207 & -4.017607088289 \\
\hline $\mathrm{C}$ & 2.652118297102 & -3.274519771424 & -1.409795652646 \\
\hline $\mathrm{H}$ & 2.078502998347 & -3.280060543743 & 0.623378182143 \\
\hline $\mathrm{H}$ & 3.056840869970 & -3.161332768947 & -3.535181613680 \\
\hline $\mathrm{H}$ & 3.537733622656 & -3.863295035223 & -1.206428752121 \\
\hline $\mathrm{H}$ & -1.061239292079 & -0.844073745721 & -3.235495553219 \\
\hline $\mathrm{H}$ & -1.330866914896 & 2.562259866651 & -0.619769122364 \\
\hline $\mathrm{N}$ & 3.391931778372 & -0.354752070309 & 1.362293475585 \\
\hline $\mathrm{H}$ & 4.285066304406 & -0.733714393025 & 1.085769340846 \\
\hline $\mathrm{H}$ & 3.271718918558 & -0.152873523059 & 2.340825874123 \\
\hline $\mathrm{H}$ & -1.852597461569 & 2.725432810861 & 1.751283413491 \\
\hline $\mathrm{H}$ & 1.621881234834 & 0.790013300847 & 2.888566026794 \\
\hline $\mathrm{C}$ & -0.461765924627 & 1.984564065927 & 3.808211807692 \\
\hline $\mathrm{C}$ & -1.370577026955 & 0.855347192449 & 4.232001176454 \\
\hline $\mathrm{H}$ & -0.966851290488 & 2.941551029973 & 3.941280661668 \\
\hline $\mathrm{H}$ & 0.440647898267 & 1.990492185573 & 4.418681851592 \\
\hline $\mathrm{C}$ & -2.753430038029 & 0.978032345862 & 4.123532703161 \\
\hline $\mathrm{C}$ & -0.832876654833 & -0.340629001306 & 4.702814478501 \\
\hline $\mathrm{C}$ & -3.585124652505 & -0.081928530893 & 4.463490777201 \\
\hline $\mathrm{H}$ & -3.180165025543 & 1.911646258661 & 3.773615077407 \\
\hline $\mathrm{C}$ & -1.660313885400 & -1.404789889825 & 5.036741010130 \\
\hline $\mathrm{H}$ & 0.242951126232 & -0.438374247201 & 4.804069506599 \\
\hline $\mathrm{C}$ & 5645240 & 27601085 & 4.914017992430 \\
\hline $\mathrm{H}$ & -4.659319737448 & 0.025860765485 & 4.376648624459 \\
\hline $\mathrm{H}$ & -1.230456902288 & -2.331033987929 & 5.397797895234 \\
\hline $\mathrm{H}$ & -3.686588422461 & -2.105148329094 & 5.177834115765 \\
\hline & NAH: $\quad$ G50a & & \\
\hline $\mathrm{H}$ & 1.090026616093 & 2.520270354077 & -0.656638316371 \\
\hline $\mathrm{C}$ & 0.433529859826 & 2.861802026130 & 0.155894008983 \\
\hline $\mathrm{C}$ & -0.828016495476 & 3.449833703697 & -0.418731165315 \\
\hline $\mathrm{H}$ & 1.006364356430 & 3.639573915635 & 0.678556180068 \\
\hline $\mathrm{C}$ & 0.093522396239 & 1.720841343647 & 1.090587220091 \\
\hline $\mathrm{C}$ & -2.048370387843 & 3.050849421889 & -0.060939964395 \\
\hline $\mathrm{C}$ & -1.181873230904 & 1.397180057312 & 1.384983435025 \\
\hline $\mathrm{C}$ & 1.148272524379 & 0.920620688153 & 1.737501139100 \\
\hline $\mathrm{N}$ & -2.265289635956 & 2.034681003630 & 0.866535153979 \\
\hline $\mathrm{O}$ & 0.923676999446 & -0.041847975131 & 2.474621893533 \\
\hline $\mathrm{H}$ & 0.100663311239 & 1.153901600146 & -2.838552834889 \\
\hline $\mathrm{C}$ & -0.054818866431 & 0.356078176592 & -2.122221743995 \\
\hline $\mathrm{C}$ & 1.093820695895 & -0.210747405514 & -1.500495779549 \\
\hline $\mathrm{C}$ & -1.296516234532 & -0.107458970817 & -1.827309305588 \\
\hline $\mathrm{C}$ & 0.938282627560 & -1.241469382534 & -0.529928687280 \\
\hline $\mathrm{C}$ & 2.370347822946 & 0.219234858992 & -1.828480354480 \\
\hline $\mathrm{C}$ & -1.439281486457 & -1.166128622499 & -0.896951947421 \\
\hline $\mathrm{H}$ & -2.174212113823 & 0.312099241212 & -2.301085477683 \\
\hline $\mathrm{C}$ & -0.367150670005 & -1.718490464668 & -0.255298815875 \\
\hline $\mathrm{N}$ & 2.040727413873 & -1.745641234305 & 0.094961291317 \\
\hline $\mathrm{C}$ & 3.488544199657 & -0.372869513784 & -1.256971287178 \\
\hline $\mathrm{H}$ & -2.428527736684 & -1.555598795657 & -0.690325764575 \\
\hline
\end{tabular}




$\begin{array}{llll}\mathrm{H} & -0.529629111348 & -2.535194700450 & 0.429362570794 \\ \mathrm{C} & 3.304979994392 & -1.389739687100 & -0.278210318312 \\ \mathrm{C} & 1.863557678571 & -2.698768701846 & 1.200676705922 \\ \mathrm{C} & 4.801853461244 & 0.029558741548 & -1.622500483172 \\ \mathrm{C} & 4.451816627935 & -2.007378645209 & 0.276882217309 \\ \mathrm{H} & 2.662552743504 & -2.561064049883 & 1.918683570916 \\ \mathrm{H} & 0.935502852864 & -2.477963776422 & 1.710506650169 \\ \mathrm{H} & 1.867901007846 & -3.718899815433 & 0.817319013790 \\ \mathrm{C} & 5.886780215734 & -0.564594886691 & -1.058952993100 \\ \mathrm{H} & 4.903134847859 & 0.814216452714 & -2.362038020436 \\ \mathrm{C} & 5.696881032534 & -1.598918222656 & -0.112158165768 \\ \mathrm{H} & 4.362007720346 & -2.818543446168 & 0.981060307440 \\ \mathrm{H} & 6.889050284647 & -0.265308445580 & -1.333849561328 \\ \mathrm{H} & 6.562559774809 & -2.089695981653 & 0.314300968359 \\ \mathrm{H} & 2.497861964890 & 1.012998421727 & -2.557083813415 \\ \mathrm{H} & -0.742274877428 & 4.244747329883 & -1.148166190406 \\ \mathrm{~N} & 2.418039938447 & 1.297140999047 & 1.457994439702 \\ \mathrm{H} & 2.634568290979 & 2.109494647565 & 0.904414578397 \\ \mathrm{H} & 3.178517640306 & 0.799276986005 & 1.895757655147 \\ \mathrm{H} & -2.947073936005 & 3.493521232802 & -0.470250892438 \\ \mathrm{H} & -1.392584396161 & 0.587486706038 & 2.073861007878 \\ \mathrm{C} & -3.611242170134 & 1.683625539597 & 1.309903452514 \\ \mathrm{C} & -4.140108351292 & 0.416078935586 & 0.682925884299 \\ \mathrm{H} & -4.260948512774 & 2.522538203709 & 1.061980553978 \\ \mathrm{H} & -3.599786744882 & 1.579127599148 & 2.395474139263 \\ \mathrm{C} & -4.553840803283 & 0.409473325033 & -0.648258249991 \\ \mathrm{C} & -4.216355802864 & -0.760558430633 & 1.421356173241 \\ \mathrm{C} & -5.033219242789 & -0.754630642046 & -1.231332999415 \\ \mathrm{H} & -4.502022277570 & 1.325581926987 & -1.227330022057 \\ \mathrm{C} & -4.699336468631 & -1.929104181377 & 0.841247816825 \\ \mathrm{H} & -3.901853654222 & -0.759025649629 & 2.459176538153 \\ \mathrm{C} & -5.108351227283 & -1.927699280529 & -0.485555476887 \\ \mathrm{H} & -5.354513378148 & -0.748335005383 & -2.265626523617 \\ \mathrm{H} & -4.757071491456 & -2.838322654814 & 1.426777385798 \\ \mathrm{H} & -5.486495596112 & -2.835418870088 & -0.938966797056\end{array}$

$\begin{array}{llll}\text { MA+BNAH: } \quad \text { G51a } & & \\ \mathrm{H} & 1.067651439150 & -0.218162403046 & -2.779983724661 \\ \mathrm{C} & 0.092869188323 & 0.212133955539 & -2.510679813216 \\ \mathrm{C} & -0.902693281247 & -0.894706894743 & -2.291372063512 \\ \mathrm{H} & -0.201732009379 & 0.818643274778 & -3.377209798922 \\ \mathrm{C} & 0.202350441044 & 1.064800529288 & -1.265603332311 \\ \mathrm{C} & -1.600565602466 & -1.035366656771 & -1.166579931432 \\ \mathrm{C} & -0.566680406136 & 0.846689928438 & -0.179603165561 \\ \mathrm{C} & 1.161893927128 & 2.183019600783 & -1.183187447788 \\ \mathrm{~N} & -1.458011700423 & -0.177044027654 & -0.073980014191 \\ \mathrm{O} & 1.288713859440 & 2.895462323393 & -0.188368525523 \\ \mathrm{H} & 2.481995938268 & -2.782871568200 & -2.454362534853 \\ \mathrm{C} & 1.901455791359 & -2.825526302127 & -1.541052257509 \\ \mathrm{C} & 2.090034261769 & -1.788850474912 & -0.585878912694 \\ \mathrm{C} & 1.032030969885 & -3.840596053505 & -1.294912048433 \\ \mathrm{C} & 1.336333362839 & -1.790339747472 & 0.621870976607 \\ \mathrm{C} & 3.016186415967 & -0.776832438980 & -0.804141752901 \\ \mathrm{C} & 0.311996460939 & -3.854952704048 & -0.077029924878 \\ \mathrm{H} & 0.891262586923 & -4.637676122374 & -2.012276024058 \\ \mathrm{C} & 0.442258069954 & -2.864786784917 & 0.855580945084 \\ \mathrm{~N} & 1.510722931792 & -0.774303723352 & 1.517720457840 \\ \mathrm{C} & 3.250066610710 & 0.192909219210 & 0.158458914902 \\ \mathrm{H} & -0.362704425830 & -4.676426949928 & 0.128179913136 \\ \mathrm{H} & -0.113595885081 & -2.941774289216 & 1.775703275211 \\ \mathrm{C} & 2.477769387322 & 0.174791926088 & 1.353302800936 \\ \mathrm{C} & 0.644134351970 & -0.674253158504 & 2.700736668899\end{array}$




$\begin{array}{llll}\mathrm{C} & 4.238665533134 & 1.197829593570 & -0.028974879017 \\ \mathrm{C} & 2.747771965395 & 1.141446876978 & 2.351141610393 \\ \mathrm{H} & 1.164944972661 & -1.054928637584 & 3.578825840244 \\ \mathrm{H} & 0.375073565652 & 0.367562420017 & 2.848316244569 \\ \mathrm{H} & -0.267385161268 & -1.232181803644 & 2.538697685058 \\ \mathrm{C} & 4.471541079293 & 2.121325469504 & 0.939104893470 \\ \mathrm{H} & 4.796266719375 & 1.191833511292 & -0.957277317587 \\ \mathrm{C} & 3.721661453110 & 2.075242207017 & 2.138928949138 \\ \mathrm{H} & 2.215496532375 & 1.137815448506 & 3.288939301180 \\ \mathrm{H} & 5.226547359404 & 2.884586075658 & 0.807873816318 \\ \mathrm{H} & 3.926518444546 & 2.800199231407 & 2.916596238872 \\ \mathrm{H} & 3.581260827442 & -0.760147408388 & -1.730205677874 \\ \mathrm{H} & -1.053425025998 & -1.617603160212 & -3.082852366822 \\ \mathrm{~N} & 1.928161278422 & 2.378187236288 & -2.283553731588 \\ \mathrm{H} & 1.808401656499 & 1.851773259460 & -3.132875822451 \\ \mathrm{H} & 2.584905852270 & 3.143291718146 & -2.283268712093 \\ \mathrm{H} & -0.481892875211 & 1.494014813789 & 0.686050700293 \\ \mathrm{H} & -2.315037413282 & -1.835873513874 & -1.021256839000 \\ \mathrm{C} & -2.429084396634 & -0.236723537147 & 1.000478079643 \\ \mathrm{C} & -3.803860711375 & 0.269378682445 & 0.614750049610 \\ \mathrm{H} & -2.039011876994 & 0.355485565517 & 1.830359838815 \\ \mathrm{H} & -2.508700194614 & -1.269933726564 & 1.348180708840 \\ \mathrm{C} & -3.986848571410 & 1.146651312460 & -0.449354381565 \\ \mathrm{C} & -4.910941409237 & -0.133295791465 & 1.358539196233 \\ \mathrm{C} & -5.256911916190 & 1.619622669647 & -0.760565648134 \\ \mathrm{H} & -3.136223158444 & 1.461252631748 & -1.043215837008 \\ \mathrm{C} & -6.178541060642 & 0.340467194268 & 1.051343169889 \\ \mathrm{H} & -4.775732774826 & -0.825641768373 & 2.182361288132 \\ \mathrm{C} & -6.355197747066 & 1.219696402868 & -0.011311703398 \\ \mathrm{H} & -5.386699817418 & 2.300409642792 & -1.592791765102 \\ \mathrm{H} & -7.030775344318 & 0.018615530152 & 1.637053215971 \\ \mathrm{H} & -7.344255468868 & 1.586025395957 & -0.256115825202\end{array}$

Coordinates of the geometries in Table S8 (corresponding total energies are listed in Table S8) MA+_HEH(Me): G17

$\begin{array}{llll}\mathrm{H} & 0.868193676902 & -1.657042345294 & -0.834921203468 \\ \mathrm{C} & 0.949881465958 & -1.755678644452 & 0.250625507894 \\ \mathrm{C} & 2.069917906362 & -0.884817011756 & 0.768964090872 \\ \mathrm{H} & 1.186345821427 & -2.813557307417 & 0.412765756206 \\ \mathrm{C} & -0.381214148526 & -1.429438220608 & 0.890141402748 \\ \mathrm{C} & 1.889075378488 & -0.028926957114 & 1.800558922406 \\ \mathrm{C} & 3.384295643942 & -1.003016935489 & 0.133618694803 \\ \mathrm{C} & -0.484903717156 & -0.573788223298 & 1.934107040975 \\ \mathrm{C} & -1.504451904103 & -2.167278449340 & 0.309181020941 \\ \mathrm{~N} & 0.641834453482 & 0.086343935308 & 2.371621840718 \\ \mathrm{C} & 2.928793783676 & 0.844080763014 & 2.433304801313 \\ \mathrm{O} & 4.411502784103 & -0.444558028625 & 0.458775451614 \\ \mathrm{O} & 3.344647917285 & -1.851675680873 & -0.909422350269 \\ \mathrm{C} & -1.714237023235 & -0.237340234581 & 2.727566709645 \\ \mathrm{O} & -1.356126487536 & -2.989242438080 & -0.572857014479 \\ \mathrm{O} & -2.714534387580 & -1.878682408026 & 0.809524232246 \\ \mathrm{H} & 0.543019775737 & 0.711872529030 & 3.157187582654 \\ \mathrm{H} & 3.655587030441 & 0.236532619974 & 2.973906990594 \\ \mathrm{H} & 3.478407344773 & 1.404406156499 & 1.681925710043 \\ \mathrm{H} & 2.461440925409 & 1.537327867415 & 3.132459956598 \\ \mathrm{C} & 4.567205513774 & -2.014599091600 & -1.624085537178 \\ \mathrm{H} & -2.154380007759 & -1.137417166961 & 3.152990935234 \\ \mathrm{H} & -1.459926404551 & 0.444975274928 & 3.537863912643 \\ \mathrm{H} & -2.475276158264 & 0.226977820988 & 2.101993298593 \\ \mathrm{C} & -3.796064224209 & -2.674781547743 & 0.325074658897 \\ \mathrm{H} & 4.900114104544 & -1.061839729477 & -2.035931076710 \\ \mathrm{H} & 5.343298253673 & -2.415639624557 & -0.972752343839\end{array}$




\begin{tabular}{|c|c|c|c|}
\hline $\mathrm{H}$ & 4.353065486647 & -2.715389467768 & -2.426594901307 \\
\hline $\mathrm{H}$ & -4.696746311374 & -2.249390826989 & 0.760498334007 \\
\hline $\mathrm{H}$ & -3.850321997229 & -2.641948602367 & -0.761109617316 \\
\hline $\mathrm{H}$ & -3.675249622312 & -3.708718341065 & 0.650232419875 \\
\hline $\mathrm{H}$ & 1.786059245867 & 0.073079991978 & -2.984291270114 \\
\hline $\mathrm{C}$ & 1.694410299008 & 0.803306540872 & -2.189531496377 \\
\hline $\mathrm{C}$ & 0.413174323641 & 0.988179982315 & -1.600255934998 \\
\hline $\mathrm{C}$ & 2.758037606720 & 1.537455151302 & -1.768340902260 \\
\hline $\mathrm{C}$ & 0.252796329957 & 1.913566617074 & -0.530774150409 \\
\hline $\mathrm{C}$ & -0.693226305432 & 0.283417506821 & -2.051999422824 \\
\hline $\mathrm{C}$ & 2.579803465407 & 2.488445964394 & -0.735916016478 \\
\hline $\mathrm{H}$ & 3.733951019813 & 1.408809037932 & -2.216755311472 \\
\hline $\mathrm{C}$ & 1.373815146289 & 2.674227123817 & -0.119518120848 \\
\hline $\mathrm{N}$ & -0.973274444858 & 2.062248840388 & 0.052455591798 \\
\hline $\mathrm{C}$ & -1.952424443310 & 0.518571062087 & -1.517011568309 \\
\hline $\mathrm{H}$ & 3.423373094506 & 3.090486961335 & -0.422066177567 \\
\hline $\mathrm{H}$ & 1.287458842015 & 3.425488000857 & 0.649091347166 \\
\hline $\mathrm{C}$ & -2.087371897644 & 1.453410109697 & -0.451127210615 \\
\hline $\mathrm{C}$ & -1.091990343331 & 2.917737344634 & 1.242782340875 \\
\hline $\mathrm{C}$ & -3.101390915348 & -0.148205155064 & -2.023497420194 \\
\hline $\mathrm{C}$ & -3.384969549768 & 1.731789602947 & 0.042239826107 \\
\hline $\mathrm{H}$ & -1.912217041501 & 2.566613402597 & 1.856365735799 \\
\hline $\mathrm{H}$ & -0.184244507341 & 2.834842872374 & 1.830060078451 \\
\hline $\mathrm{H}$ & -1.257307618259 & 3.953386244301 & 0.946892255655 \\
\hline $\mathrm{C}$ & -4.333720600698 & 0.123885653634 & -1.519563140536 \\
\hline $\mathrm{H}$ & -2.959028357346 & -0.865374511116 & -2.822549492188 \\
\hline $\mathrm{C}$ & -4.464871042805 & 1.081913023335 & -0.487229250742 \\
\hline $\mathrm{H}$ & -3.543610588625 & 2.472961280328 & 0.809168640642 \\
\hline $\mathrm{H}$ & -5.212646849701 & -0.376679077591 & -1.902819174516 \\
\hline $\mathrm{H}$ & -5.451055251969 & 1.317121121308 & -0.107257044674 \\
\hline $\mathrm{H}$ & -0.577590803089 & -0.441792823893 & -2.850644407859 \\
\hline \multicolumn{4}{|c|}{$\mathrm{MA}+\_\mathrm{HEH}(\mathrm{Me}): \quad$ G19 } \\
\hline $\mathrm{H}$ & -0.000078013626 & 1.478714296064 & -0.785673322156 \\
\hline $\mathrm{C}$ & 0.000015547843 & 1.770365361994 & 0.268531983344 \\
\hline $\mathrm{C}$ & -1.257532633376 & 1.267810521696 & 0.939645679196 \\
\hline $\mathrm{H}$ & -0.000010530553 & 2.865669361669 & 0.245511635402 \\
\hline $\mathrm{C}$ & 1.257712429370 & 1.267866251093 & 0.939408733814 \\
\hline $\mathrm{C}$ & -1.217892988434 & 0.535291561274 & 2.076959375165 \\
\hline $\mathrm{C}$ & -2.544575655549 & 1.620729434360 & 0.333900772537 \\
\hline $\mathrm{C}$ & 1.218319765424 & 0.535347120574 & 2.076730622825 \\
\hline $\mathrm{C}$ & 2.544625979438 & 1.620837953552 & 0.333419347348 \\
\hline $\mathrm{N}$ & 0.000272155644 & 0.207677176898 & 2.624342251786 \\
\hline $\mathrm{C}$ & -2.392780072096 & 0.013854562222 & 2.846134217340 \\
\hline $\mathrm{O}$ & -3.651792369963 & 1.391739358879 & 0.773543333386 \\
\hline $\mathrm{O}$ & -2.367668307495 & 2.285050440525 & -0.823387583800 \\
\hline $\mathrm{C}$ & 2.393375328665 & 0.013963346947 & 2.845684195731 \\
\hline $\mathrm{O}$ & 3.651935173357 & 1.391905658720 & 0.772858832043 \\
\hline $\mathrm{O}$ & 2.367472267593 & 2.285136923723 & -0.823844010408 \\
\hline $\mathrm{H}$ & 0.000364123320 & -0.341197076385 & 3.471480374501 \\
\hline $\mathrm{H}$ & -2.934922458143 & 0.837563091948 & 3.312134157741 \\
\hline $\mathrm{H}$ & -3.093354679010 & -0.496178147622 & 2.189890130942 \\
\hline $\mathrm{H}$ & -2.058511389194 & -0.670977739052 & 3.625174111112 \\
\hline $\mathrm{C}$ & -3.557510440738 & 2.676896708318 & -1.503255221625 \\
\hline $\mathrm{H}$ & 2.935570817829 & 0.837696280017 & 3.311578923184 \\
\hline $\mathrm{H}$ & 2.059283857700 & -0.670881496843 & 3.624788922890 \\
\hline $\mathrm{H}$ & 3.093846894101 & -0.496041477143 & 2.189308438198 \\
\hline $\mathrm{C}$ & 3.557170170736 & 2.677031009916 & -1.503936846069 \\
\hline $\mathrm{H}$ & -3.231690969900 & 3.165027015437 & -2.418062498897 \\
\hline $\mathrm{H}$ & -4.170748151590 & 1.806985259546 & -1.737567196087 \\
\hline $\mathrm{H}$ & -4.135534467148 & 3.370809647267 & -0.893045370111 \\
\hline $\mathrm{H}$ & 3.231158066598 & 3.165142012431 & -2.418685794972 \\
\hline
\end{tabular}




\begin{tabular}{|c|c|c|c|}
\hline $\mathrm{H}$ & 4.135278780428 & 3.370972014854 & -0.893839159579 \\
\hline $\mathrm{H}$ & 4.170400740756 & 1.807144199358 & -1.738358944259 \\
\hline $\mathrm{H}$ & -2.421806304132 & 0.010131119813 & -3.058360837665 \\
\hline $\mathrm{C}$ & -2.447775755813 & -0.618477715177 & -2.176376933137 \\
\hline $\mathrm{C}$ & -1.207104392669 & -0.999500526289 & -1.595466714195 \\
\hline $\mathrm{C}$ & -3.620745058455 & -1.037914220594 & -1.633374919403 \\
\hline $\mathrm{C}$ & -1.193191723382 & -1.794839682780 & -0.414704820921 \\
\hline $\mathrm{C}$ & -0.000118256772 & -0.598887461267 & -2.151535862703 \\
\hline $\mathrm{C}$ & -3.596835818064 & -1.869213908802 & -0.488787137564 \\
\hline $\mathrm{H}$ & -4.568373872863 & -0.752673195693 & -2.069823514223 \\
\hline $\mathrm{C}$ & -2.427955787835 & -2.242483458754 & 0.113047714236 \\
\hline $\mathrm{N}$ & 0.000104611776 & -2.112734420537 & 0.168905886906 \\
\hline $\mathrm{C}$ & 1.206975762932 & -0.999467603454 & -1.595677213798 \\
\hline $\mathrm{H}$ & -4.532026538328 & -2.225149272557 & -0.075195563378 \\
\hline $\mathrm{H}$ & -2.464310388452 & -2.896718063889 & 0.969394797193 \\
\hline $\mathrm{C}$ & 1.193290425023 & -1.794806420068 & -0.414912593572 \\
\hline $\mathrm{C}$ & 0.000228283105 & -2.815670219720 & 1.460730875610 \\
\hline $\mathrm{C}$ & 2.447535256331 & -0.618412223528 & -2.176804578757 \\
\hline $\mathrm{C}$ & 2.428158537197 & -2.242415390848 & 0.112625826368 \\
\hline $\mathrm{H}$ & 0.874499516150 & -2.517393539274 & 2.027947174133 \\
\hline $\mathrm{H}$ & -0.873961744874 & -2.517434312087 & 2.028093534756 \\
\hline $\mathrm{H}$ & 0.000240232192 & -3.893892906211 & 1.302745520699 \\
\hline $\mathrm{C}$ & 3.620610755830 & -1.037816248557 & -1.634006690511 \\
\hline $\mathrm{H}$ & 2.421394751109 & 0.010194222768 & -3.058785130609 \\
\hline $\mathrm{C}$ & 3.596923534434 & -1.869115287956 & -0.489413844414 \\
\hline $\mathrm{H}$ & 2.464680248188 & -2.896647901223 & 0.968967558840 \\
\hline $\mathrm{H}$ & 4.568155951566 & -0.752551492856 & -2.070621523231 \\
\hline $\mathrm{H}$ & 4.532195980752 & -2.225025137762 & -0.075985160777 \\
\hline $\mathrm{H}$ & -0.000204176930 & 0.023664635065 & -3.040322940406 \\
\hline \multicolumn{4}{|c|}{$\mathrm{MA}+\_\mathrm{HEH}(\mathrm{Me}): \quad \mathrm{G} 25$} \\
\hline $\mathrm{H}$ & -0.782107619558 & 1.117227700353 & -0.819352849726 \\
\hline $\mathrm{C}$ & -1.360265087402 & 0.376250025823 & -1.377415712825 \\
\hline $\mathrm{C}$ & -0.633415273899 & -0.947718254606 & -1.391685715925 \\
\hline $\mathrm{H}$ & -1.423532986364 & 0.785621046413 & -2.392302673075 \\
\hline $\mathrm{C}$ & -2.741431434166 & 0.274436686595 & -0.774365930517 \\
\hline $\mathrm{C}$ & -1.251741437544 & -2.102097724168 & -1.050652087574 \\
\hline $\mathrm{C}$ & 0.766656949924 & -0.957521816255 & -1.818196441443 \\
\hline $\mathrm{C}$ & -3.297644635891 & -0.916606934812 & -0.455399191682 \\
\hline $\mathrm{C}$ & -3.376404962023 & 1.575432618088 & -0.554570309918 \\
\hline $\mathrm{N}$ & -2.562627956914 & -2.066623660701 & -0.638738623992 \\
\hline $\mathrm{C}$ & -0.659315975211 & -3.477399438854 & -1.071215185148 \\
\hline $\mathrm{O}$ & 1.466872510355 & -1.925364681180 & -2.035199286602 \\
\hline $\mathrm{O}$ & 1.235902638237 & 0.296879352461 & -1.965513877938 \\
\hline $\mathrm{C}$ & -4.656105194693 & -1.169547982739 & 0.129115179140 \\
\hline $\mathrm{O}$ & -2.839942658501 & 2.621200079456 & -0.861077004252 \\
\hline $\mathrm{O}$ & -4.585610694850 & 1.546182280688 & 0.022274359484 \\
\hline $\mathrm{H}$ & -2.992942334973 & -2.941708038209 & -0.378917551977 \\
\hline $\mathrm{H}$ & -0.577127198063 & -3.834275475963 & -2.099450500617 \\
\hline $\mathrm{H}$ & 0.341286670195 & -3.481316632017 & -0.648632409050 \\
\hline $\mathrm{H}$ & -1.292870632123 & -4.166982692540 & -0.512907404665 \\
\hline $\mathrm{C}$ & 2.559420702185 & 0.419837564565 & -2.483601205375 \\
\hline $\mathrm{H}$ & -5.434384735891 & -0.694605252882 & -0.462845817237 \\
\hline $\mathrm{H}$ & -4.849081104154 & -2.240596172457 & 0.169990900946 \\
\hline $\mathrm{H}$ & -4.719860097591 & -0.765162647642 & 1.139248257457 \\
\hline $\mathrm{C}$ & -5.204054236828 & 2.815008929880 & 0.236828412460 \\
\hline $\mathrm{H}$ & 3.252068525924 & -0.235480782402 & -1.958624246502 \\
\hline $\mathrm{H}$ & 2.567827877105 & 0.167653708732 & -3.544938258994 \\
\hline $\mathrm{H}$ & 2.841247425016 & 1.461440441518 & -2.346013594772 \\
\hline $\mathrm{H}$ & -6.164418203987 & 2.601388130585 & 0.698317404683 \\
\hline $\mathrm{H}$ & -4.597055965266 & 3.430142019389 & 0.900320702353 \\
\hline $\mathrm{H}$ & -5.348822404427 & 3.336236693372 & -0.708837599735 \\
\hline
\end{tabular}




\begin{tabular}{|c|c|c|c|}
\hline $\mathrm{H}$ & 2.078385038237 & 3.882923755409 & 0.619917149342 \\
\hline $\mathrm{C}$ & 2.767614376291 & 3.050860689103 & 0.548842914013 \\
\hline $\mathrm{C}$ & 2.267829491908 & 1.747986112529 & 0.820885054164 \\
\hline $\mathrm{C}$ & 4.074704052744 & 3.233175345875 & 0.222337418179 \\
\hline $\mathrm{C}$ & 3.133017240581 & 0.623278389265 & 0.715735225407 \\
\hline $\mathrm{C}$ & 0.943902919729 & 1.546492624269 & 1.190331303936 \\
\hline $\mathrm{C}$ & 4.940044098390 & 2.114613226065 & 0.170710351627 \\
\hline $\mathrm{H}$ & 4.462753153860 & 4.221897565831 & 0.018573685669 \\
\hline $\mathrm{C}$ & 4.496130265485 & 0.843165907513 & 0.403936674876 \\
\hline $\mathrm{N}$ & 2.630993194677 & -0.631611533154 & 0.915970179182 \\
\hline $\mathrm{C}$ & 0.479414745532 & 0.278213162601 & 1.505949782362 \\
\hline $\mathrm{H}$ & 5.987800015013 & 2.267509144121 & -0.054993887555 \\
\hline $\mathrm{H}$ & 5.198985306015 & 0.025736743592 & 0.372951827218 \\
\hline $\mathrm{C}$ & 1.361977021087 & -0.832539252375 & 1.379080011868 \\
\hline $\mathrm{C}$ & 3.480186076684 & -1.798499146333 & 0.633271358117 \\
\hline $\mathrm{C}$ & -0.859979496219 & 0.069837325948 & 1.937339968605 \\
\hline $\mathrm{C}$ & 0.894248026642 & -2.115108276883 & 1.751297689375 \\
\hline $\mathrm{H}$ & 2.854303474930 & -2.616632799823 & 0.297540776983 \\
\hline $\mathrm{H}$ & 4.159658312385 & -1.555890531990 & -0.175153076867 \\
\hline $\mathrm{H}$ & 4.038030168252 & -2.083263389699 & 1.525081401098 \\
\hline $\mathrm{C}$ & -1.290639400351 & -1.175765497758 & 2.270549740611 \\
\hline $\mathrm{H}$ & -1.512586328418 & 0.932851594634 & 1.997278439875 \\
\hline $\mathrm{C}$ & -0.392896827956 & -2.265726276662 & 2.187763233085 \\
\hline $\mathrm{H}$ & 1.544682611607 & -2.974897367714 & 1.727192468470 \\
\hline $\mathrm{H}$ & -2.307605742357 & -1.340884474780 & 2.601313446410 \\
\hline $\mathrm{H}$ & -0.730996436361 & -3.252120486735 & 2.480299377052 \\
\hline $\mathrm{H}$ & 0.270388172993 & 2.394609356657 & 1.254526749917 \\
\hline \multicolumn{4}{|c|}{ MA+_HEH(Me): G26 } \\
\hline $\mathrm{H}$ & 0.187101529294 & 1.0093068778666 & 1.028367737025 \\
\hline $\mathrm{C}$ & 0.979135363331 & 0.422305944123 & 1.491619050026 \\
\hline $\mathrm{C}$ & 0.548729176251 & -1.023848592315 & 1.576838554814 \\
\hline $\mathrm{H}$ & 1.101155564170 & 0.847601804045 & 2.495603942867 \\
\hline $\mathrm{C}$ & 2.251326224755 & 0.571605640356 & 0.688953534793 \\
\hline $\mathrm{C}$ & 1.429383385107 & -2.029517139609 & 1.365359158057 \\
\hline $\mathrm{C}$ & -0.834868506144 & -1.318665483992 & 1.947675790492 \\
\hline $\mathrm{C}$ & 3.082534071402 & -0.477559948659 & 0.496722854821 \\
\hline $\mathrm{C}$ & 2.549202436348 & 1.872648884858 & 0.089067655798 \\
\hline $\mathrm{N}$ & 2.707134798049 & -1.721093736098 & 0.954389654933 \\
\hline $\mathrm{C}$ & 1.169074603386 & -3.494637314859 & 1.532886210266 \\
\hline $\mathrm{O}$ & -1.370860705857 & -2.408510644529 & 1.975482821494 \\
\hline $\mathrm{O}$ & -1.504023078039 & -0.197398909546 & 2.277711677453 \\
\hline $\mathrm{C}$ & 4.390999080689 & -0.464957545401 & -0.227756048699 \\
\hline $\mathrm{O}$ & 3.441756288734 & 2.135593007828 & -0.690173133471 \\
\hline $\mathrm{O}$ & 1.667988314134 & 2.809163281254 & 0.490619871066 \\
\hline $\mathrm{H}$ & 3.338977976309 & -2.489914767575 & 0.783312869117 \\
\hline $\mathrm{H}$ & 0.709478533558 & -3.697655564451 & 2.497974484514 \\
\hline $\mathrm{H}$ & 0.477314881037 & -3.849798045342 & 0.769231458450 \\
\hline $\mathrm{H}$ & 2.100823574315 & -4.054258032813 & 1.453750019287 \\
\hline $\mathrm{C}$ & -2.845927110983 & -0.377002326145 & 2.727946871178 \\
\hline $\mathrm{H}$ & 4.978000400134 & 0.413002882862 & 0.025655908079 \\
\hline $\mathrm{H}$ & 4.958201021392 & -1.364381765416 & 0.011651150038 \\
\hline $\mathrm{H}$ & 4.217378041851 & -0.440985189147 & -1.306114670618 \\
\hline $\mathrm{C}$ & 1.837339032639 & 4.107844157779 & -0.073807958869 \\
\hline $\mathrm{H}$ & -3.225471141617 & 0.619369632595 & 2.938413553294 \\
\hline $\mathrm{H}$ & -3.456547586086 & -0.856339977321 & 1.963606933350 \\
\hline $\mathrm{H}$ & -2.863143392741 & -0.984179505826 & 3.633187190601 \\
\hline $\mathrm{H}$ & 1.051308072437 & 4.724262681944 & 0.353958541139 \\
\hline $\mathrm{H}$ & 2.814570738206 & 4.514042413986 & 0.185959994185 \\
\hline $\mathrm{H}$ & 1.741690426215 & 4.071661100208 & -1.159328417829 \\
\hline $\mathrm{H}$ & -0.965094707547 & 3.695536694727 & -1.250531377093 \\
\hline $\mathrm{C}$ & -1.739829740801 & 2.994826250922 & -0.964091012346 \\
\hline
\end{tabular}




\begin{tabular}{|c|c|c|c|}
\hline $\mathrm{C}$ & -1.485001021281 & 1.609856702377 & -1.158699688079 \\
\hline $\mathrm{C}$ & -2.915380879141 & 3.409530335529 & -0.423718963135 \\
\hline $\mathrm{C}$ & -2.471472070469 & 0.644565843060 & -0.802483552234 \\
\hline $\mathrm{C}$ & -0.265398301572 & 1.178514481791 & -1.663376749214 \\
\hline $\mathrm{C}$ & -3.883845337665 & 2.446099603951 & -0.055193815045 \\
\hline $\mathrm{H}$ & -3.114728959164 & 4.460535634009 & -0.264966681933 \\
\hline $\mathrm{C}$ & -3.684890812044 & 1.106184408601 & -0.237293215946 \\
\hline $\mathrm{N}$ & -2.208660596260 & -0.682730381493 & -1.003494751490 \\
\hline $\mathrm{C}$ & 0.017101518636 & -0.172886001862 & -1.781370918642 \\
\hline $\mathrm{H}$ & -4.812801917944 & 2.775729967146 & 0.392525129684 \\
\hline $\mathrm{H}$ & -4.451569162558 & 0.422896442601 & 0.087897183057 \\
\hline $\mathrm{C}$ & -0.987883150102 & -1.117698455892 & -1.431878444627 \\
\hline $\mathrm{C}$ & -3.250833462639 & -1.700834837014 & -0.794219398994 \\
\hline $\mathrm{C}$ & 1.294094326927 & -0.625367150829 & -2.215605288730 \\
\hline $\mathrm{C}$ & -0.685494527520 & -2.497540431974 & -1.524179949636 \\
\hline $\mathrm{H}$ & -3.026130337495 & -2.278174399501 & 0.101836081337 \\
\hline $\mathrm{H}$ & -4.219480220641 & -1.232093365095 & -0.705320113328 \\
\hline $\mathrm{H}$ & -3.271075117233 & -2.353477815641 & -1.662957806803 \\
\hline $\mathrm{C}$ & 1.562647928519 & -1.955353171394 & -2.290859349123 \\
\hline $\mathrm{H}$ & 2.038093934203 & 0.122025352647 & -2.465459344169 \\
\hline $\mathrm{C}$ & 0.557439421373 & -2.888075978875 & -1.937582666206 \\
\hline $\mathrm{H}$ & -1.409225759648 & -3.246626872645 & -1.244624123786 \\
\hline $\mathrm{H}$ & 2.534185922070 & -2.308237575481 & -2.610299523197 \\
\hline $\mathrm{H}$ & 0.780827079795 & -3.946594858011 & -1.986698342101 \\
\hline $\mathrm{H}$ & 0.482884937926 & 1.910812757682 & -1.951293575876 \\
\hline \multicolumn{4}{|c|}{$\mathrm{MA}+\_\mathrm{HEH}(\mathrm{Me}): \quad$ G27 } \\
\hline $\mathrm{H}$ & -0.596772873535 & 0.878931777010 & -1.156122859507 \\
\hline $\mathrm{C}$ & -1.297549241953 & 0.083928521378 & -1.412578363460 \\
\hline $\mathrm{C}$ & -0.596663142613 & -1.252250534186 & -1.363088268795 \\
\hline $\mathrm{H}$ & -1.623998917871 & 0.312399203276 & -2.435109855110 \\
\hline $\mathrm{C}$ & -2.466767372122 & 0.136826930817 & -0.457588379222 \\
\hline $\mathrm{C}$ & -1.221904273695 & -2.348685528935 & -0.875033503133 \\
\hline $\mathrm{C}$ & 0.757468276168 & -1.354488525691 & -1.904928888888 \\
\hline $\mathrm{C}$ & -3.041846476202 & -0.996104268384 & 0.007082151016 \\
\hline $\mathrm{C}$ & -2.872470251092 & 1.491892408339 & -0.081815489659 \\
\hline $\mathrm{N}$ & -2.472541311774 & -2.208524225010 & -0.318034456984 \\
\hline $\mathrm{C}$ & -0.695355716617 & -3.750552486933 & -0.870274622779 \\
\hline $\mathrm{O}$ & 1.480666366372 & -2.330404496255 & -1.896042812808 \\
\hline $\mathrm{O}$ & 1.154437110747 & -0.190576073955 & -2.454327274751 \\
\hline $\mathrm{C}$ & -4.231405184704 & -1.122224045963 & 0.910162122154 \\
\hline $\mathrm{O}$ & -2.378829142723 & 2.485215489999 & -0.578015970627 \\
\hline $\mathrm{O}$ & -3.807952993598 & 1.574404477197 & 0.874768497924 \\
\hline $\mathrm{H}$ & -2.912750068940 & -3.039900993242 & 0.048065707229 \\
\hline $\mathrm{H}$ & -0.421145861636 & -4.061143034558 & -1.877371995342 \\
\hline $\mathrm{H}$ & 0.204164592158 & -3.820778621814 & -0.260732611599 \\
\hline $\mathrm{H}$ & -1.446621282971 & -4.434493396474 & -0.476442586915 \\
\hline $\mathrm{C}$ & 2.433397515071 & -0.202737766124 & -3.086169827591 \\
\hline $\mathrm{H}$ & -5.042277858809 & -0.470797293679 & 0.598126810989 \\
\hline $\mathrm{H}$ & -4.586276604308 & -2.152635337588 & 0.917661463791 \\
\hline $\mathrm{H}$ & -3.957307406678 & -0.844855444067 & 1.930009067316 \\
\hline $\mathrm{C}$ & -4.203036267072 & 2.895412037287 & 1.246418504211 \\
\hline $\mathrm{H}$ & 3.213452520547 & -0.504829588762 & -2.388382545042 \\
\hline $\mathrm{H}$ & 2.428692606942 & -0.887606644731 & -3.934555942745 \\
\hline $\mathrm{H}$ & 2.607610696342 & 0.814365734676 & -3.427053645486 \\
\hline $\mathrm{H}$ & -4.964680307300 & 2.772028324165 & 2.011549224767 \\
\hline $\mathrm{H}$ & -3.354742665739 & 3.450249453707 & 1.646399763360 \\
\hline $\mathrm{H}$ & -4.613076008728 & 3.428790891914 & 0.389545194959 \\
\hline $\mathrm{H}$ & 0.790344524380 & 4.060536434793 & 0.299509445455 \\
\hline $\mathrm{C}$ & 1.624486174066 & 3.416442098732 & 0.049733883217 \\
\hline $\mathrm{C}$ & 1.569636782714 & 2.064026322799 & 0.484098189688 \\
\hline $\mathrm{C}$ & 2.688670802235 & 3.869429230632 & -0.663264781064 \\
\hline
\end{tabular}




\begin{tabular}{|c|c|c|c|}
\hline $\mathrm{C}$ & 2.639972902883 & 1.172769459664 & 0.182202080069 \\
\hline $\mathrm{C}$ & 0.469617782638 & 1.590776388511 & 1.188333804529 \\
\hline $\mathrm{C}$ & 3.740402445559 & 2.976562973939 & -0.974779533189 \\
\hline $\mathrm{H}$ & 2.735925577508 & 4.895714570704 & -1.000975838576 \\
\hline $\mathrm{C}$ & 3.730826653382 & 1.670678317944 & -0.571295802704 \\
\hline $\mathrm{N}$ & 2.570494777326 & -0.123827869788 & 0.613525107625 \\
\hline $\mathrm{C}$ & 0.387606416304 & 0.260432592303 & 1.568243389202 \\
\hline $\mathrm{H}$ & 4.579331947240 & 3.331911715545 & -1.559672098639 \\
\hline $\mathrm{H}$ & 4.548721954699 & 1.035298525601 & -0.867859773911 \\
\hline $\mathrm{C}$ & 1.467242995782 & -0.612061373545 & 1.253512693843 \\
\hline $\mathrm{C}$ & 3.707119997731 & -1.041662008470 & 0.434068279716 \\
\hline $\mathrm{C}$ & -0.756537331769 & -0.245767279059 & 2.245713601708 \\
\hline $\mathrm{C}$ & 1.361308487237 & -1.977945423149 & 1.607045028770 \\
\hline $\mathrm{H}$ & 3.893505265048 & -1.547411673363 & 1.377631383593 \\
\hline $\mathrm{H}$ & 3.472203023620 & -1.769443130006 & -0.341760831439 \\
\hline $\mathrm{H}$ & 4.597100348731 & -0.488797916759 & 0.173058141846 \\
\hline $\mathrm{C}$ & -0.830226200249 & -1.560519031866 & 2.584679000710 \\
\hline $\mathrm{H}$ & -1.557267928362 & 0.447553657845 & 2.473998186197 \\
\hline $\mathrm{C}$ & 0.239988649536 & -2.423551144739 & 2.250342337488 \\
\hline $\mathrm{H}$ & 2.139400802025 & -2.680384837806 & 1.354031997278 \\
\hline $\mathrm{H}$ & -1.698550371747 & -1.954150010045 & 3.096095594864 \\
\hline $\mathrm{H}$ & 0.168355931979 & -3.474286736735 & 2.502783488054 \\
\hline $\mathrm{H}$ & -0.343995864164 & 2.268318202902 & 1.426340418401 \\
\hline \multicolumn{4}{|c|}{$\mathrm{MA}+$ HEH(Me): G28 } \\
\hline $\mathrm{H}$ & -0.367722535610 & 0.271983912502 & -1.057273237798 \\
\hline $\mathrm{C}$ & 0.540902151121 & 0.773419656751 & -1.396912599902 \\
\hline $\mathrm{C}$ & 0.673986158580 & 2.116812432668 & -0.719437775366 \\
\hline $\mathrm{H}$ & 0.400909675346 & 0.899951238491 & -2.477515321585 \\
\hline $\mathrm{C}$ & 1.729456484578 & -0.122576269447 & -1.128548300368 \\
\hline $\mathrm{C}$ & 1.853590585075 & 2.533614175355 & -0.204716808693 \\
\hline $\mathrm{C}$ & -0.498511590812 & 2.991341809479 & -0.669026825534 \\
\hline $\mathrm{C}$ & 2.880294107525 & 0.362116184789 & -0.605883218457 \\
\hline $\mathrm{C}$ & 1.502088031691 & -1.528011488890 & -1.462746073418 \\
\hline $\mathrm{N}$ & 2.937625823062 & 1.688267870004 & -0.237690161145 \\
\hline $\mathrm{C}$ & 2.131868801570 & 3.855216075802 & 0.442640262518 \\
\hline $\mathrm{O}$ & -0.584367824800 & 4.073361834784 & -0.125831654934 \\
\hline $\mathrm{O}$ & -1.540308203524 & 2.451380456413 & -1.329549873513 \\
\hline $\mathrm{C}$ & 4.155942250159 & -0.381644799779 & -0.343253920606 \\
\hline $\mathrm{O}$ & 0.427776309248 & -1.941845582394 & -1.856276033239 \\
\hline $\mathrm{O}$ & 2.551056677247 & -2.345797726644 & -1.300202451540 \\
\hline $\mathrm{H}$ & 3.798090666198 & 2.020503294129 & 0.171797465190 \\
\hline $\mathrm{H}$ & 1.792753252616 & 4.676767209197 & -0.183786806001 \\
\hline $\mathrm{H}$ & 1.598144399392 & 3.934226803554 & 1.390259106997 \\
\hline $\mathrm{H}$ & 3.199686923553 & 3.961382662194 & 0.630997093525 \\
\hline $\mathrm{C}$ & -2.717224801572 & 3.254835683282 & -1.392189968053 \\
\hline $\mathrm{H}$ & 4.542853598203 & -0.825295205867 & -1.258553568090 \\
\hline $\mathrm{H}$ & 4.905537664614 & 0.295415963333 & 0.064342132189 \\
\hline $\mathrm{H}$ & 3.992715189035 & -1.192282314345 & 0.365302865539 \\
\hline $\mathrm{C}$ & 2.308171866759 & -3.727729647552 & -1.561484875452 \\
\hline $\mathrm{H}$ & -3.063920226058 & 3.515590209604 & -0.393156360109 \\
\hline $\mathrm{H}$ & -2.520998441089 & 4.169118170352 & -1.952694227607 \\
\hline $\mathrm{H}$ & -3.463153865583 & 2.654381461223 & -1.906151664198 \\
\hline $\mathrm{H}$ & 3.245867340839 & -4.237274570470 & -1.355976610424 \\
\hline $\mathrm{H}$ & 1.522077720612 & -4.108762931134 & -0.908708634763 \\
\hline $\mathrm{H}$ & 2.019084917289 & -3.877941188268 & -2.601123037884 \\
\hline $\mathrm{H}$ & 0.676912989412 & 1.555248593320 & 2.678751749024 \\
\hline $\mathrm{C}$ & 0.797926417617 & 0.514863049448 & 2.401792619479 \\
\hline $\mathrm{C}$ & -0.299765179496 & -0.133788045180 & 1.769741647219 \\
\hline $\mathrm{C}$ & 1.952500973432 & -0.159828891389 & 2.642361837226 \\
\hline $\mathrm{C}$ & -0.189050005926 & -1.499674783586 & 1.377572981461 \\
\hline $\mathrm{C}$ & -1.480028394040 & 0.548922692349 & 1.514654246676 \\
\hline
\end{tabular}




$\begin{array}{llll}\mathrm{C} & 2.056890054184 & -1.515062069214 & 2.246132315242 \\ \mathrm{H} & 2.791557681342 & 0.326668410871 & 3.121664698775 \\ \mathrm{C} & 1.033540880056 & -2.172824614705 & 1.624925920715 \\ \mathrm{~N} & -1.247056067215 & -2.109028304962 & 0.768635953878 \\ \mathrm{C} & -2.525970839670 & -0.067566770516 & 0.842892947612 \\ \mathrm{H} & 2.982698111027 & -2.047379580202 & 2.425034654804 \\ \mathrm{H} & 1.187949712754 & -3.191398416483 & 1.306959214676 \\ \mathrm{C} & -2.378726966771 & -1.423386010024 & 0.435909357482 \\ \mathrm{C} & -1.185858060950 & -3.541591975514 & 0.444552944773 \\ \mathrm{C} & -3.721124843031 & 0.637451423325 & 0.533072183472 \\ \mathrm{C} & -3.416583250316 & -2.020612073621 & -0.320252860000 \\ \mathrm{H} & -0.902325688096 & -3.667774131019 & -0.599319230389 \\ \mathrm{H} & -0.473970808355 & -4.033915404142 & 1.092628525864 \\ \mathrm{H} & -2.158059385153 & -3.983555724782 & 0.633880259415 \\ \mathrm{C} & -4.711317809898 & 0.035999841679 & -0.176848478378 \\ \mathrm{H} & -3.807038704688 & 1.661034778589 & 0.875219347147 \\ \mathrm{C} & -4.539268561776 & -1.299469930421 & -0.614401039604 \\ \mathrm{H} & -3.324808488744 & -3.027662658780 & -0.694151554846 \\ \mathrm{H} & -5.620802541105 & 0.568107872575 & -0.420988130712 \\ \mathrm{H} & -5.317996859456 & -1.765610609953 & -1.204629089556 \\ \mathrm{H} & -1.578441901936 & 1.584433623080 & 1.825855999495\end{array}$

$\begin{array}{llll}\mathrm{MA}+\mathrm{HEH}(\mathrm{Me}) \mathrm{G} \text { G5 } & & \\ \mathrm{H} & 0.215041637635 & -0.485271288790 & -1.172698955346 \\ \mathrm{C} & 0.938147180708 & 0.267492587209 & -1.494844745543 \\ \mathrm{C} & 0.319805789856 & 1.640464265303 & -1.372120224728 \\ \mathrm{H} & 1.138153276831 & 0.030032170476 & -2.547549494860 \\ \mathrm{C} & 2.198523479433 & 0.136310149295 & -0.673855755500 \\ \mathrm{C} & 1.037975800877 & 2.692410820541 & -0.913585902318 \\ \mathrm{C} & -1.082222574527 & 1.710100488377 & -1.780965605871 \\ \mathrm{C} & 2.866642204246 & 1.227654330530 & -0.237351549330 \\ \mathrm{C} & 2.620563124576 & -1.245108832137 & -0.438336633388 \\ \mathrm{~N} & 2.321827447139 & 2.472751070994 & -0.465261514085 \\ \mathrm{C} & 0.603565134325 & 4.123333274226 & -0.800296672031 \\ \mathrm{O} & -1.707193066464 & 0.734076783322 & -2.150862919152 \\ \mathrm{O} & -1.650359266974 & 2.922055323283 & -1.714974524463 \\ \mathrm{C} & 4.163557348870 & 1.277232425421 & 0.514294647259 \\ \mathrm{O} & 2.103462936774 & -2.192027325751 & -0.995514507616 \\ \mathrm{O} & 3.599175482899 & -1.406380334512 & 0.464696764577 \\ \mathrm{H} & 2.836405709334 & 3.270597289098 & -0.123607408630 \\ \mathrm{H} & 0.233305365068 & 4.497862552211 & -1.751654596377 \\ \mathrm{H} & -0.204634019637 & 4.220518297510 & -0.075264409890 \\ \mathrm{H} & 1.438557874649 & 4.743897008027 & -0.477496412270 \\ \mathrm{C} & -3.033017672030 & 2.980514056472 & -2.063484198257 \\ \mathrm{H} & 4.004438956191 & 1.009899917411 & 1.560276236619 \\ \mathrm{H} & 4.890874671970 & 0.584288379708 & 0.102154759069 \\ \mathrm{H} & 4.574455843578 & 2.286055533000 & 0.477539452962 \\ \mathrm{C} & 4.018371932068 & -2.752301200510 & 0.692036479174 \\ \mathrm{H} & -3.621853808939 & 2.342182653008 & -1.404063674731 \\ \mathrm{H} & -3.326163087554 & 4.019583564759 & -1.938917619890 \\ \mathrm{H} & -3.183317667606 & 2.668765815200 & -3.096571894036 \\ \mathrm{H} & 4.811203077374 & -2.694536234945 & 1.432871350900 \\ \mathrm{H} & 3.191314493496 & -3.351786607568 & 1.072171358495 \\ \mathrm{H} & 4.394386614455 & -3.198105461307 & -0.228208161218 \\ \mathrm{H} & 0.604342842606 & -4.064524436407 & 1.004968209073 \\ \mathrm{C} & -0.350375193193 & -3.757680958837 & 0.597160090650 \\ \mathrm{C} & -0.761914889607 & -2.413665235426 & 0.810399767118 \\ \mathrm{C} & -1.133189095475 & -4.612167069064 & -0.111820506271 \\ \mathrm{C} & -2.010788284822 & -1.965607700183 & 0.296999912154 \\ \mathrm{C} & 0.044021570153 & -1.512888508482 & 1.491137820026 \\ \mathrm{C} & -2.358262829390 & -4.148657953128 & -0.649899821352 \\ \mathrm{H} & -0.825273492001 & -5.634716580060 & -0.282882980534 \\ & & & \end{array}$




\begin{tabular}{|c|c|c|c|}
\hline $\mathrm{C}$ & -2.797933801283 & -2.869540282685 & -0.457202880193 \\
\hline $\mathrm{N}$ & -2.417562224827 & -0.684173303984 & 0.530380497214 \\
\hline $\mathrm{C}$ & -0.339942703544 & -0.188262108285 & 1.641791785253 \\
\hline $\mathrm{H}$ & -2.964830517768 & -4.824557133700 & -1.239286013228 \\
\hline $\mathrm{H}$ & -3.726204920260 & -2.557483575539 & -0.907813715354 \\
\hline $\mathrm{C}$ & -1.606635946988 & 0.229243835917 & 1.138158087287 \\
\hline $\mathrm{C}$ & -3.769709315607 & -0.302876013618 & 0.097640381553 \\
\hline $\mathrm{C}$ & 0.520226022979 & 0.754859370537 & 2.271271475688 \\
\hline $\mathrm{C}$ & -1.968533062193 & 1.593061513584 & 1.277862193026 \\
\hline $\mathrm{H}$ & -4.106680827051 & 0.557188100183 & 0.658929324478 \\
\hline $\mathrm{H}$ & -3.762320210947 & -0.081893881630 & -0.968520616428 \\
\hline $\mathrm{H}$ & -4.447508015070 & -1.122235118330 & 0.313424987576 \\
\hline $\mathrm{C}$ & 0.143714438238 & 2.054036442517 & 2.400007556176 \\
\hline $\mathrm{H}$ & 1.476617409185 & 0.399156388075 & 2.635625105655 \\
\hline $\mathrm{C}$ & -1.114585225117 & 2.462363912750 & 1.895026940068 \\
\hline $\mathrm{H}$ & -2.894917053337 & 1.973652082704 & 0.878514958991 \\
\hline $\mathrm{H}$ & 0.793702239917 & 2.776937425787 & 2.874681857843 \\
\hline $\mathrm{H}$ & -1.408390511283 & 3.500511709114 & 1.986677002452 \\
\hline $\mathrm{H}$ & 0.996150378059 & -1.844056391670 & 1.894195911556 \\
\hline \multicolumn{4}{|c|}{$\mathrm{MA}+$ HEH(Me): G6 } \\
\hline $\mathrm{H}$ & -0.736355766490 & -1.581805968214 & -1.226636311915 \\
\hline $\mathrm{C}$ & -0.856188726840 & -1.862975934525 & -0.176298525290 \\
\hline $\mathrm{C}$ & 0.422073935056 & -1.582915801559 & 0.578392864086 \\
\hline $\mathrm{H}$ & -1.044398332110 & -2.942636538122 & -0.203072192539 \\
\hline $\mathrm{C}$ & -2.048783924633 & -1.143868414956 & 0.406835241271 \\
\hline $\mathrm{C}$ & 0.421435278783 & -0.916974860564 & 1.757085269431 \\
\hline $\mathrm{C}$ & 1.612024748326 & -2.162020512783 & -0.047902737719 \\
\hline $\mathrm{C}$ & -1.979044488907 & -0.487762885972 & 1.587430680856 \\
\hline $\mathrm{C}$ & -3.258542508230 & -1.255449117313 & -0.410613779147 \\
\hline $\mathrm{N}$ & -0.766670272311 & -0.416217867109 & 2.240198382374 \\
\hline $\mathrm{C}$ & 1.589145691524 & -0.649608503639 & 2.662469107403 \\
\hline $\mathrm{O}$ & 1.552937957869 & -2.835278985564 & -1.057088927335 \\
\hline $\mathrm{O}$ & 2.777854952067 & -1.907000063146 & 0.564079977132 \\
\hline $\mathrm{C}$ & -3.092646790671 & 0.196341553224 & 2.325559962363 \\
\hline $\mathrm{O}$ & -3.298833281379 & -1.914356025416 & -1.429683247866 \\
\hline $\mathrm{O}$ & -4.324928999964 & -0.572897083401 & 0.030713327816 \\
\hline $\mathrm{H}$ & -0.746481736544 & 0.047811936495 & 3.135792475938 \\
\hline $\mathrm{H}$ & 2.048472951190 & -1.584494327799 & 2.979904427298 \\
\hline $\mathrm{H}$ & 2.360417361758 & -0.070879404933 & 2.156762832304 \\
\hline $\mathrm{H}$ & 1.261444000693 & -0.102705346743 & 3.545850851158 \\
\hline $\mathrm{C}$ & 3.921822748295 & -2.574258712554 & 0.030434525293 \\
\hline $\mathrm{H}$ & -3.908409916523 & -0.497102834194 & 2.521635849423 \\
\hline $\mathrm{H}$ & -2.729218043026 & 0.585979093343 & 3.275964313927 \\
\hline $\mathrm{H}$ & -3.498662018577 & 1.020385456454 & 1.742278923674 \\
\hline $\mathrm{C}$ & -5.495322847930 & -0.657702990561 & -0.781110078569 \\
\hline $\mathrm{H}$ & 4.031058943790 & -2.374599105679 & -1.033372123976 \\
\hline $\mathrm{H}$ & 4.776211426952 & -2.183344133476 & 0.577215139341 \\
\hline $\mathrm{H}$ & 3.835963290225 & -3.649745554669 & 0.189821209817 \\
\hline $\mathrm{H}$ & -6.239088300159 & -0.031421199809 & -0.295581262666 \\
\hline $\mathrm{H}$ & -5.292692975941 & -0.290793947802 & -1.786993825285 \\
\hline $\mathrm{H}$ & -5.849867635885 & -1.686493890665 & -0.837602013830 \\
\hline $\mathrm{H}$ & 3.167533508385 & -0.307801066818 & -2.851921493624 \\
\hline $\mathrm{C}$ & 3.257690236063 & 0.258979616290 & -1.933423912767 \\
\hline $\mathrm{C}$ & 2.068847879147 & 0.790550181165 & -1.362892777524 \\
\hline $\mathrm{C}$ & 4.461346620528 & 0.471419624663 & -1.340257319756 \\
\hline $\mathrm{C}$ & 2.131543017088 & 1.523680487130 & -0.142816581026 \\
\hline $\mathrm{C}$ & 0.840355866675 & 0.616426027944 & -1.984381858895 \\
\hline $\mathrm{C}$ & 4.521484765412 & 1.231795144189 & -0.149153898807 \\
\hline $\mathrm{H}$ & 5.370209451744 & 0.072421558361 & -1.769966931678 \\
\hline $\mathrm{C}$ & 3.401770223855 & 1.745291076394 & 0.443360843602 \\
\hline $\mathrm{N}$ & 0.976842257737 & 1.996446616671 & 0.413481392778 \\
\hline
\end{tabular}




$\begin{array}{llll}\mathrm{C} & -0.308607336197 & 1.190861263667 & -1.460396034396 \\ \mathrm{H} & 5.484380792476 & 1.422739064185 & 0.307708553574 \\ \mathrm{H} & 3.512101464299 & 2.339904820089 & 1.335907575653 \\ \mathrm{C} & -0.222578812765 & 1.908649130018 & -0.235083511427 \\ \mathrm{C} & 1.007568684678 & 2.641829572586 & 1.734743752761 \\ \mathrm{C} & -1.558508661238 & 1.076549367503 & -2.128509253982 \\ \mathrm{C} & -1.388627319851 & 2.536632671270 & 0.264826011506 \\ \mathrm{H} & 0.123217206031 & 2.346750395026 & 2.290802882130 \\ \mathrm{H} & 1.871592946469 & 2.300412725784 & 2.288151407781 \\ \mathrm{H} & 1.036401074313 & 3.725031816765 & 1.620490312754 \\ \mathrm{C} & -2.666052333520 & 1.681773438128 & -1.623416985135 \\ \mathrm{H} & -1.591236657379 & 0.503223587997 & -3.046755049854 \\ \mathrm{C} & -2.563815775465 & 2.426831739109 & -0.425298141443 \\ \mathrm{H} & -1.361960673039 & 3.127630803472 & 1.166629589217 \\ \mathrm{H} & -3.619888721107 & 1.604376357605 & -2.128102724470 \\ \mathrm{H} & -3.443196691923 & 2.926591314023 & -0.038424013299 \\ \mathrm{H} & 0.782011571293 & 0.044597316613 & -2.904484003290\end{array}$

Coordinates of the geometries in Table S9 (corresponding total energies are listed in Table S9) MA+ TMBIH: G14

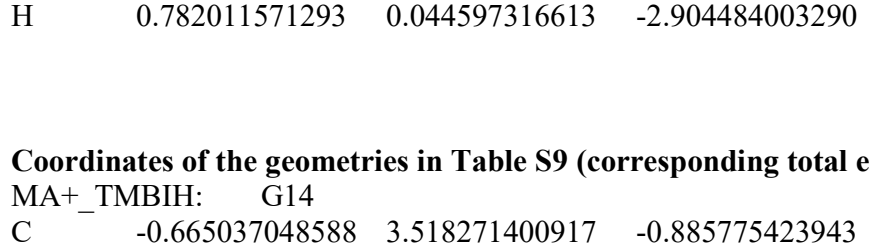

\begin{tabular}{|c|c|c|c|}
\hline $\mathrm{C}$ & -0.665037048588 & 3.518271400917 & -0.885775423943 \\
\hline $\mathrm{C}$ & 0.705175124927 & 3.693887180788 & -0.863494623755 \\
\hline $\mathrm{C}$ & -1.276072773281 & 2.486478879675 & -0.146994780322 \\
\hline $\mathrm{H}$ & -1.284760920150 & 4.187246514721 & -1.469692483457 \\
\hline $\mathrm{C}$ & 1.533421256502 & 2.848356065277 & -0.099051979559 \\
\hline $\mathrm{H}$ & 1.154476476087 & 4.500125986410 & -1.429640874300 \\
\hline $\mathrm{C}$ & -0.463364074736 & 1.651835488211 & 0.589321802911 \\
\hline $\mathrm{H}$ & -2.351989934120 & 2.360536617861 & -0.149106259536 \\
\hline $\mathrm{C}$ & 0.936470489062 & 1.832729745169 & 0.616225206893 \\
\hline $\mathrm{H}$ & 2.605264975899 & 2.999810668117 & -0.067136635707 \\
\hline $\mathrm{N}$ & -0.761012401060 & 0.549210941719 & 1.369923630731 \\
\hline $\mathrm{N}$ & 1.477403879350 & 0.844579268616 & 1.420822534397 \\
\hline $\mathrm{C}$ & 0.392707812282 & 0.292409415573 & 2.238896224138 \\
\hline $\mathrm{C}$ & -2.097241613371 & 0.331743148374 & 1.866890213213 \\
\hline $\mathrm{C}$ & 2.800150040284 & 0.988988736944 & 1.977063046145 \\
\hline $\mathrm{H}$ & 0.529706150130 & -0.787656419107 & 2.360205818663 \\
\hline $\mathrm{H}$ & -2.134937024338 & -0.623053192543 & 2.392541273533 \\
\hline $\mathrm{H}$ & -2.430140943530 & 1.124709172331 & 2.547870367823 \\
\hline $\mathrm{H}$ & -2.790823010714 & 0.283510778036 & 1.024958575939 \\
\hline $\mathrm{H}$ & 3.056546186643 & 0.089393931543 & 2.536799443976 \\
\hline $\mathrm{H}$ & 3.522759388315 & 1.101989908072 & 1.167039651414 \\
\hline $\mathrm{H}$ & 2.885960001527 & 1.857472742950 & 2.642153038069 \\
\hline $\mathrm{H}$ & 2.063359676304 & -3.249362511730 & 1.196088706026 \\
\hline $\mathrm{C}$ & 2.129772048442 & -2.478535699401 & 0.438071674360 \\
\hline $\mathrm{C}$ & 0.918734232403 & -2.020232674013 & -0.151776559499 \\
\hline $\mathrm{C}$ & 3.323715850961 & -1.959539937134 & 0.050686865888 \\
\hline $\mathrm{C}$ & 0.956493811465 & -0.996965889974 & -1.138002472000 \\
\hline $\mathrm{C}$ & -0.307650436686 & -2.544901759054 & 0.222019639100 \\
\hline $\mathrm{C}$ & 3.355515595709 & -0.964185926462 & -0.957163737037 \\
\hline $\mathrm{H}$ & 4.248236107325 & -2.301054610215 & 0.496450657277 \\
\hline $\mathrm{C}$ & 2.217328551126 & -0.489134597380 & -1.542384301541 \\
\hline $\mathrm{N}$ & -0.205396827225 & -0.526581679275 & -1.675731087258 \\
\hline $\mathrm{C}$ & -1.484418755259 & -2.069831972166 & -0.340593438990 \\
\hline $\mathrm{H}$ & 4.310845069061 & -0.564235243491 & -1.273219021671 \\
\hline $\mathrm{H}$ & 2.294897929154 & 0.269798021719 & -2.304291049361 \\
\hline $\mathrm{C}$ & -1.423895183062 & -1.021555966615 & -1.305613338499 \\
\hline $\mathrm{C}$ & -0.084721680053 & 0.544224479191 & -2.676968062948 \\
\hline $\mathrm{C}$ & -2.741574103901 & -2.603449593656 & 0.054695648254 \\
\hline $\mathrm{C}$ & -2.645816873563 & -0.536824185286 & -1.836514756505 \\
\hline $\mathrm{H}$ & 0.485840924803 & 1.364381988148 & -2.246790015851 \\
\hline
\end{tabular}




\begin{tabular}{|c|c|c|c|}
\hline $\mathrm{H}$ & 0.422954174418 & 0.150387914611 & -3.556052204443 \\
\hline $\mathrm{H}$ & -1.054389595030 & 0.917968091093 & -2.963334556027 \\
\hline $\mathrm{C}$ & -3.896136228481 & -2.122475233087 & -0.476269440444 \\
\hline $\mathrm{H}$ & -2.742896132537 & -3.397363107997 & 0.791184011958 \\
\hline $\mathrm{C}$ & -3.832434597661 & -1.078904251461 & -1.427863690510 \\
\hline $\mathrm{H}$ & -2.674556582103 & 0.252284964930 & -2.568173607685 \\
\hline $\mathrm{H}$ & -4.855437084986 & -2.523696924896 & -0.178960483095 \\
\hline $\mathrm{H}$ & -4.750833943226 & -0.691438557286 & -1.850294783617 \\
\hline $\mathrm{H}$ & -0.350736504065 & -3.331714976046 & 0.967412199852 \\
\hline $\mathrm{C}$ & 0.273748777818 & 0.957112185602 & 3.605792865418 \\
\hline $\mathrm{H}$ & -0.572418634983 & 0.542706456071 & 4.155318162314 \\
\hline $\mathrm{H}$ & 1.175155763146 & 0.776654483992 & 4.192829552520 \\
\hline $\mathrm{H}$ & 0.131918613567 & 2.035091731610 & 3.495360856746 \\
\hline \multicolumn{4}{|c|}{ MA+_TMBIH: $\quad$ G26 } \\
\hline $\mathrm{C}$ & 4.243635337373 & -1.015195673154 & -1.399070052567 \\
\hline $\mathrm{C}$ & 4.877024521686 & -0.137233311898 & -0.539984943325 \\
\hline $\mathrm{C}$ & 2.868881235393 & -0.893011278245 & -1.674666095331 \\
\hline $\mathrm{H}$ & 4.809700544277 & -1.804665028121 & -1.877297236984 \\
\hline $\mathrm{C}$ & 4.167651881680 & 0.908075127363 & 0.081472360948 \\
\hline $\mathrm{H}$ & 5.937798028726 & -0.240832044005 & -0.349220059659 \\
\hline $\mathrm{C}$ & 2.171516136978 & 0.119069220005 & -1.050937368333 \\
\hline $\mathrm{H}$ & 2.374736171001 & -1.576019420487 & -2.354662894782 \\
\hline $\mathrm{C}$ & 2.818784346196 & 1.019283906979 & -0.178809673078 \\
\hline $\mathrm{H}$ & 4.669836494283 & 1.605475252024 & 0.740108662418 \\
\hline $\mathrm{N}$ & 0.828253371022 & 0.448096176452 & -1.080867473710 \\
\hline $\mathrm{N}$ & 1.867912276120 & 1.897636233448 & 0.311253640928 \\
\hline $\mathrm{C}$ & 0.687724701295 & 1.809259967869 & -0.554506225678 \\
\hline $\mathrm{C}$ & -0.024265225742 & 0.002762669970 & -2.156430512302 \\
\hline $\mathrm{C}$ & 2.253094544720 & 3.170046573598 & 0.873006134091 \\
\hline $\mathrm{H}$ & -0.223550679469 & 1.880032807082 & 0.050090257474 \\
\hline $\mathrm{H}$ & -1.053373835983 & 0.301510560929 & -1.949413972251 \\
\hline $\mathrm{H}$ & 0.271786489056 & 0.413371434315 & -3.129537894506 \\
\hline $\mathrm{H}$ & 0.009428696987 & -1.086486450278 & -2.217477799190 \\
\hline $\mathrm{H}$ & 1.368759766486 & 3.673288097512 & 1.263990290885 \\
\hline $\mathrm{H}$ & 2.943434641135 & 3.004709557631 & 1.700755385314 \\
\hline $\mathrm{H}$ & 2.739164518486 & 3.826436706514 & 0.140876946226 \\
\hline $\mathrm{H}$ & 0.724270998924 & 0.323431184488 & 2.970936882600 \\
\hline $\mathrm{C}$ & 0.594857170264 & -0.472141754597 & 2.247624882030 \\
\hline $\mathrm{C}$ & -0.651445647566 & -0.548694632326 & 1.562965797581 \\
\hline $\mathrm{C}$ & 1.580590180317 & -1.369898365325 & 1.989875654698 \\
\hline $\mathrm{C}$ & -0.869478120661 & -1.579570206655 & 0.600213943589 \\
\hline $\mathrm{C}$ & -1.648807019431 & 0.381974003662 & 1.791930669617 \\
\hline $\mathrm{C}$ & 1.362325152668 & -2.376495498007 & 1.018555533686 \\
\hline $\mathrm{H}$ & 2.531325911744 & -1.315827690874 & 2.503012658476 \\
\hline $\mathrm{C}$ & 0.181515120261 & -2.491764796466 & 0.341209043890 \\
\hline $\mathrm{N}$ & -2.065801642327 & -1.643937056586 & -0.050572144154 \\
\hline $\mathrm{C}$ & -2.835308486711 & 0.350620956830 & 1.063672229680 \\
\hline $\mathrm{H}$ & 2.160253567418 & -3.073372588971 & 0.794144273652 \\
\hline $\mathrm{H}$ & 0.088218431297 & -3.255546786722 & -0.413607181217 \\
\hline $\mathrm{C}$ & -3.025476577551 & -0.679716379291 & 0.104513139661 \\
\hline $\mathrm{C}$ & -2.347195385067 & -2.764421318851 & -0.958770793985 \\
\hline $\mathrm{C}$ & -3.839029198515 & 1.337872040517 & 1.247602261163 \\
\hline $\mathrm{C}$ & -4.202243731354 & -0.666829983712 & -0.680444440982 \\
\hline $\mathrm{H}$ & -2.125824600066 & -2.479352550938 & -1.987032310810 \\
\hline $\mathrm{H}$ & -1.757622225048 & -3.624419336201 & -0.671835828128 \\
\hline $\mathrm{H}$ & -3.392057362464 & -3.038402373101 & -0.863624623692 \\
\hline $\mathrm{C}$ & -4.971933005317 & 1.319272613933 & 0.494174931515 \\
\hline $\mathrm{H}$ & -3.667060139015 & 2.102811516498 & 1.994480716181 \\
\hline $\mathrm{C}$ & -5.137705064773 & 0.311769860215 & -0.483075348704 \\
\hline $\mathrm{H}$ & -4.364326384557 & -1.399553166929 & -1.454720025968 \\
\hline $\mathrm{H}$ & -5.737463965112 & 2.071822404377 & 0.626243040256 \\
\hline
\end{tabular}




$\begin{array}{llll}\mathrm{H} & -6.027229186227 & 0.314423627671 & -1.100187513124 \\ \mathrm{H} & -1.495753062306 & 1.161910368785 & 2.530241486972 \\ \mathrm{C} & 0.663447811308 & 2.873905002424 & -1.646267838566 \\ \mathrm{H} & -0.208751702514 & 2.739462831194 & -2.287175525438 \\ \mathrm{H} & 0.602535427063 & 3.868241160968 & -1.202349054220 \\ \mathrm{H} & 1.566911773613 & 2.813079828486 & -2.258181992844\end{array}$

Coordinates of the geometries in Table S10 (corresponding total energies are listed in Table S10) MA+ DMPBIH: G14

\begin{tabular}{|c|c|c|c|}
\hline $\mathrm{C}$ & -0.010884234547 & -1.759008646392 & 3.477164597880 \\
\hline $\mathrm{C}$ & -0.414062434042 & -0.457051372582 & 3.700217142674 \\
\hline $\mathrm{C}$ & 0.547589342431 & -2.145141656277 & 2.241666726563 \\
\hline $\mathrm{H}$ & -0.111195532592 & -2.496822735798 & 4.263194756057 \\
\hline $\mathrm{C}$ & -0.281878685345 & 0.525712978368 & 2.698834259198 \\
\hline $\mathrm{H}$ & -0.829686402270 & -0.178383957378 & 4.660444144497 \\
\hline $\mathrm{C}$ & 0.670184340868 & -1.185869582402 & 1.261301139669 \\
\hline $\mathrm{H}$ & 0.883524766474 & -3.161109738809 & 2.075341636110 \\
\hline $\mathrm{C}$ & 0.254400507791 & 0.143200284596 & 1.489401301342 \\
\hline $\mathrm{H}$ & -0.577484996652 & 1.551707810637 & 2.880559301688 \\
\hline $\mathrm{N}$ & 1.156199001173 & -1.263801217006 & -0.032989303043 \\
\hline $\mathrm{N}$ & 0.487834264025 & 0.862873029176 & 0.331237878679 \\
\hline $\mathrm{C}$ & 1.393119638577 & 0.095562398086 & -0.524192682924 \\
\hline $\mathrm{C}$ & 2.015074747127 & -2.343697026873 & -0.450986623190 \\
\hline $\mathrm{C}$ & 0.536518380717 & 2.303852382900 & 0.328371592169 \\
\hline $\mathrm{H}$ & 1.081175320329 & 0.177191312793 & -1.572209104701 \\
\hline $\mathrm{C}$ & 2.850060193063 & 0.530198167046 & -0.434189330872 \\
\hline $\mathrm{H}$ & 2.213130436306 & -2.252524858774 & -1.519241797215 \\
\hline $\mathrm{H}$ & 2.971479658126 & -2.351304327235 & 0.086899284598 \\
\hline $\mathrm{H}$ & 1.509767319657 & -3.295149615746 & -0.278119369092 \\
\hline $\mathrm{H}$ & 0.630096652608 & 2.657351413645 & -0.699154939021 \\
\hline $\mathrm{H}$ & -0.394325760570 & 2.696952918743 & 0.741132125102 \\
\hline $\mathrm{H}$ & 1.375892830934 & 2.697910437255 & 0.914402628403 \\
\hline $\mathrm{C}$ & 3.559775822260 & 0.810382576821 & -1.594663605447 \\
\hline $\mathrm{C}$ & 3.492474280185 & 0.634059611632 & 0.800231146729 \\
\hline $\mathrm{C}$ & 4.897252856529 & 1.189427302303 & -1.532773338554 \\
\hline $\mathrm{H}$ & 3.061043240926 & 0.731007472735 & -2.554701367297 \\
\hline $\mathrm{C}$ & 4.825055741754 & 1.012367793656 & 0.864830646129 \\
\hline $\mathrm{H}$ & 2.948978811902 & 0.421348392297 & 1.714175132960 \\
\hline $\mathrm{C}$ & 5.530565288396 & 1.290675831150 & -0.302794055232 \\
\hline $\mathrm{H}$ & 5.440606688716 & 1.406157813273 & -2.444107619403 \\
\hline $\mathrm{H}$ & 5.316906006798 & 1.092740654536 & 1.826238364064 \\
\hline $\mathrm{H}$ & 6.570726780253 & 1.586864114568 & -0.249440261878 \\
\hline $\mathrm{H}$ & -2.048450359243 & 3.190778157686 & -2.534657716197 \\
\hline $\mathrm{C}$ & -2.383300688437 & 2.824611287855 & -1.572073319695 \\
\hline $\mathrm{C}$ & -2.242730752915 & 1.434459153319 & -1.307905935911 \\
\hline $\mathrm{C}$ & -2.913348957223 & 3.656621412669 & -0.637921288259 \\
\hline $\mathrm{C}$ & -2.671526146575 & 0.897161500546 & -0.057994929886 \\
\hline $\mathrm{C}$ & -1.666440163007 & 0.592118145163 & -2.248562921231 \\
\hline $\mathrm{C}$ & -3.314530620868 & 3.124032469931 & 0.609074182224 \\
\hline $\mathrm{H}$ & -3.021708557715 & 4.714997583012 & -0.832288757694 \\
\hline $\mathrm{C}$ & -3.201596998672 & 1.793929404017 & 0.903656372770 \\
\hline $\mathrm{N}$ & -2.547617073959 & -0.444614318414 & 0.166596817854 \\
\hline $\mathrm{C}$ & -1.503517233675 & -0.758408786002 & -1.984834068960 \\
\hline $\mathrm{H}$ & -3.720981391874 & 3.788110102464 & 1.361372898476 \\
\hline $\mathrm{H}$ & -3.516423831746 & 1.461162626556 & 1.878064960117 \\
\hline $\mathrm{C}$ & -1.971774275473 & -1.277610169823 & -0.747283674292 \\
\hline $\mathrm{C}$ & -3.037346157100 & -1.064996207812 & 1.407162169034 \\
\hline $\mathrm{C}$ & -0.873303581862 & -1.624503139405 & -2.921395650138 \\
\hline $\mathrm{C}$ & -1.826494098998 & -2.664981130774 & -0.493091265518 \\
\hline $\mathrm{H}$ & -2.201422707654 & -1.526625498718 & 1.929319420163 \\
\hline $\mathrm{H}$ & -3.496227434680 & -0.332508368679 & 2.051345228132 \\
\hline
\end{tabular}




$\begin{array}{llll}\mathrm{H} & -3.780972392131 & -1.816649658796 & 1.149321460331 \\ \mathrm{C} & -0.727695159358 & -2.946740666676 & -2.646287942025 \\ \mathrm{H} & -0.519026037575 & -1.194214959312 & -3.850017583309 \\ \mathrm{C} & -1.219157600426 & -3.461041729027 & -1.420884137867 \\ \mathrm{H} & -2.176956992402 & -3.102001574097 & 0.427996504027 \\ \mathrm{H} & -0.246441676260 & -3.610598954198 & -3.351873460520 \\ \mathrm{H} & -1.107264705499 & -4.517158498511 & -1.209624620809 \\ \mathrm{H} & -1.328610276579 & 0.998977856083 & -3.195586147458\end{array}$

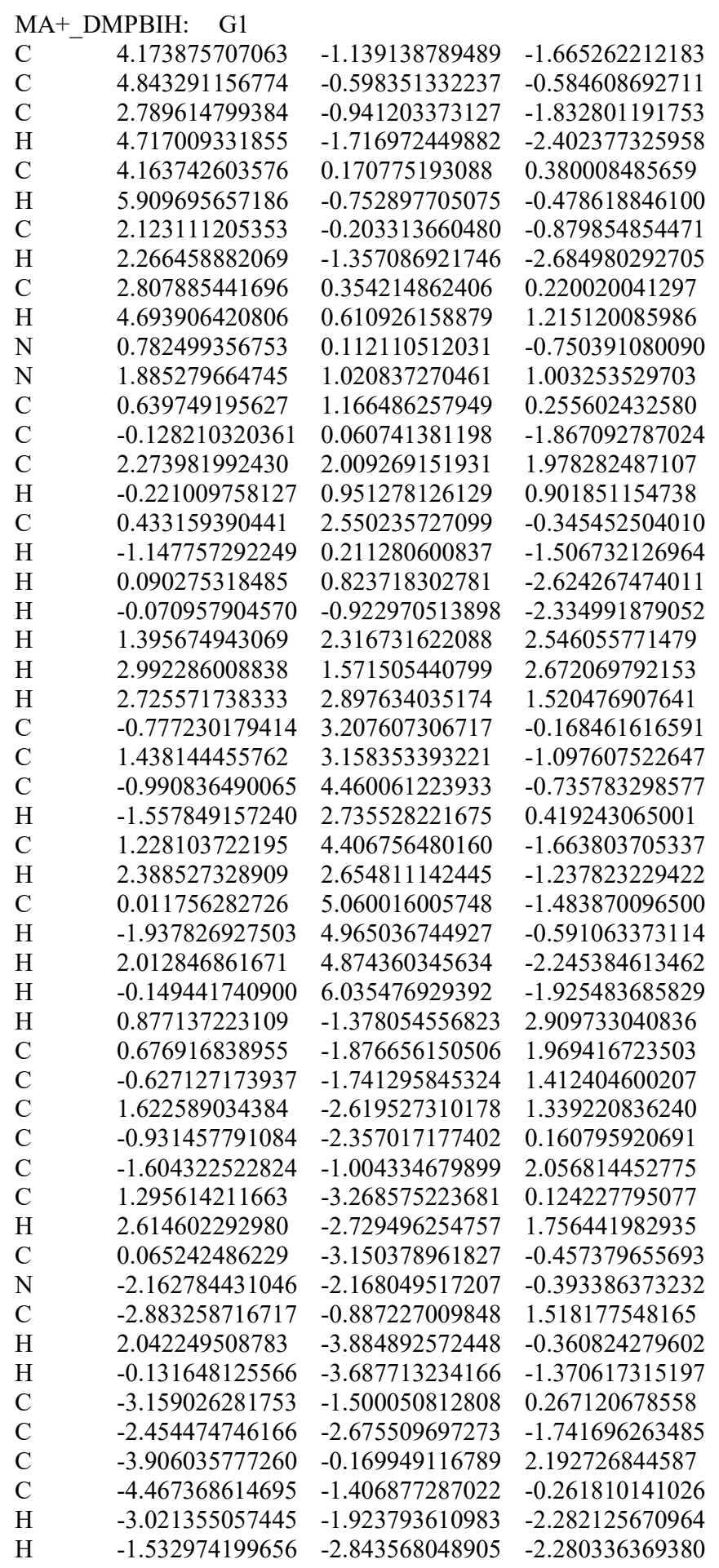




\begin{tabular}{|c|c|c|c|}
\hline $\mathrm{H}$ & -3.025722842095 & -3.600950109853 & -1.676491011366 \\
\hline $\mathrm{C}$ & -5.154016387835 & -0.080381567996 & 1.657617366681 \\
\hline $\mathrm{H}$ & -3.660844066230 & 0.292043781492 & 3.140912565550 \\
\hline $\mathrm{C}$ & -5.427745414547 & -0.716165932955 & 0.425474290399 \\
\hline $\mathrm{H}$ & -4.729600818844 & -1.893538520479 & -1.187574355585 \\
\hline $\mathrm{H}$ & -5.938118531699 & 0.461575408768 & 2.168708782787 \\
\hline $\mathrm{H}$ & -6.427220337417 & -0.662322181370 & 0.013039991350 \\
\hline $\mathrm{H}$ & -1.376709454607 & -0.522726500528 & 3.001873670360 \\
\hline \multicolumn{4}{|c|}{ MA+_DMPBIH: G20 } \\
\hline $\mathrm{C}$ & -0.669156553191 & -2.859159410120 & -2.322405684997 \\
\hline $\mathrm{C}$ & -0.431332182080 & -3.733856692024 & -1.279461056115 \\
\hline $\mathrm{C}$ & -0.325064028412 & -1.496988795123 & -2.218649887456 \\
\hline $\mathrm{H}$ & -1.113549388098 & -3.222292403714 & -3.240588242201 \\
\hline $\mathrm{C}$ & 0.165413962418 & -3.292198978879 & -0.082802037987 \\
\hline $\mathrm{H}$ & -0.691036080876 & -4.779919432384 & -1.383498276710 \\
\hline $\mathrm{C}$ & 0.236098492475 & -1.061437766377 & -1.039030920069 \\
\hline $\mathrm{H}$ & -0.494543051470 & -0.815718280597 & -3.043188938487 \\
\hline $\mathrm{C}$ & 0.483903562976 & -1.956238652652 & 0.024047619298 \\
\hline $\mathrm{H}$ & 0.370686092115 & -3.982576444723 & 0.725726134551 \\
\hline $\mathrm{N}$ & 0.616783960290 & 0.203468887909 & -0.623350204895 \\
\hline $\mathrm{N}$ & 1.043440856257 & -1.237584733684 & 1.062204647767 \\
\hline $\mathrm{C}$ & 1.464330582890 & 0.068234591903 & 0.567219343653 \\
\hline $\mathrm{C}$ & 0.855529744501 & 1.278633517480 & -1.552270236109 \\
\hline $\mathrm{C}$ & 1.720254877293 & -1.869631406419 & 2.165520670234 \\
\hline $\mathrm{H}$ & 1.215594617414 & 0.856468249637 & 1.288100262579 \\
\hline $\mathrm{C}$ & 2.951195231953 & 0.172599534154 & 0.254426473908 \\
\hline $\mathrm{H}$ & 0.924748535941 & 2.220003926872 & -1.002264505957 \\
\hline $\mathrm{H}$ & 1.774776095926 & 1.140103311163 & -2.134482605975 \\
\hline $\mathrm{H}$ & 0.013148814477 & 1.348548643191 & -2.242152831416 \\
\hline $\mathrm{H}$ & 1.958903996042 & -1.117280067883 & 2.917583181328 \\
\hline $\mathrm{H}$ & 1.058203471575 & -2.608395984919 & 2.620026045879 \\
\hline $\mathrm{H}$ & 2.647537813935 & -2.371336968339 & 1.863049710019 \\
\hline $\mathrm{C}$ & 3.642862130765 & 1.332567953390 & 0.581261008247 \\
\hline $\mathrm{C}$ & 3.620367360593 & -0.858841953682 & -0.403593422130 \\
\hline $\mathrm{C}$ & 4.987191608886 & 1.470547841029 & 0.252422922737 \\
\hline $\mathrm{H}$ & 3.123671701506 & 2.133563203562 & 1.097236769801 \\
\hline $\mathrm{C}$ & 4.961893298034 & -0.724408237958 & -0.731240665017 \\
\hline $\mathrm{H}$ & 3.092286697851 & -1.771574833422 & -0.656204843156 \\
\hline $\mathrm{C}$ & 5.647501517609 & 0.441911096125 & -0.405010890792 \\
\hline $\mathrm{H}$ & 5.517285596458 & 2.378545427878 & 0.511873861506 \\
\hline $\mathrm{H}$ & 5.476014561435 & -1.530385748786 & -1.239936669719 \\
\hline $\mathrm{H}$ & 6.694834272706 & 0.544087337856 & -0.660378764630 \\
\hline $\mathrm{H}$ & -1.566438751468 & -1.107682797408 & 3.335835431635 \\
\hline $\mathrm{C}$ & -2.039068988559 & -1.229363334502 & 2.368988268931 \\
\hline $\mathrm{C}$ & -2.012867132632 & -0.121472916381 & 1.475336913793 \\
\hline $\mathrm{C}$ & -2.643148031817 & -2.390315611004 & 2.009229341212 \\
\hline $\mathrm{C}$ & -2.595749085252 & -0.237316201435 & 0.180395574191 \\
\hline $\mathrm{C}$ & -1.415746037839 & 1.072937197815 & 1.842866181046 \\
\hline $\mathrm{C}$ & -3.258041352263 & -2.484999229297 & 0.735453903328 \\
\hline $\mathrm{H}$ & -2.669105011188 & -3.236553340860 & 2.682449443707 \\
\hline $\mathrm{C}$ & -3.239468571420 & -1.454313628030 & -0.159728467507 \\
\hline $\mathrm{N}$ & -2.528798547317 & 0.820928564100 & -0.678511530234 \\
\hline $\mathrm{C}$ & -1.420482384955 & 2.166015092774 & 0.985925341345 \\
\hline $\mathrm{H}$ & -3.762419564171 & -3.402798800879 & 0.460118735904 \\
\hline $\mathrm{H}$ & -3.744874197742 & -1.577652145960 & -1.103596839444 \\
\hline $\mathrm{C}$ & -2.018176572230 & 2.029163545065 & -0.296027249803 \\
\hline $\mathrm{C}$ & -3.006252582978 & 0.680245174353 & -2.061845477346 \\
\hline $\mathrm{C}$ & -0.829550311030 & 3.401880383757 & 1.362939663513 \\
\hline $\mathrm{C}$ & -2.062615886953 & 3.159743716967 & -1.146596007140 \\
\hline $\mathrm{H}$ & -2.315979179013 & 1.193566168081 & -2.724426276665 \\
\hline $\mathrm{H}$ & -3.013549182600 & -0.363313710440 & -2.343540608915 \\
\hline
\end{tabular}




$\begin{array}{llll}\mathrm{H} & -4.005247899912 & 1.104779133777 & -2.157293949340 \\ \mathrm{C} & -0.860171284498 & 4.466853605112 & 0.516970384929 \\ \mathrm{H} & -0.367014661410 & 3.464917384120 & 2.340216399016 \\ \mathrm{C} & -1.499736045081 & 4.336975429194 & -0.737923111621 \\ \mathrm{H} & -2.551741193958 & 3.116004776951 & -2.106655469627 \\ \mathrm{H} & -0.414687964091 & 5.411474827908 & 0.797926807038 \\ \mathrm{H} & -1.548572203735 & 5.194468175775 & -1.397053436000 \\ \mathrm{H} & -0.948828546083 & 1.159701809985 & 2.818455066368\end{array}$

Calculation of the gas-phase Gibbs free energy changes of the reactions

Procedures

The geometries of gas phase structures of the H-donors (Don-H = BNAH, HEH(Me), TIBIH and DMPBIH), their oxidized forms $\left(\mathrm{Don}^{+}=\mathrm{BNA}^{+}, \mathrm{HE}(\mathrm{Me})^{+}, \mathrm{TMBI}^{+}, \mathrm{DMPBI}^{+}\right)$, as well as acceptor $\left(\mathrm{MA}^{+}\right)$and reduced form $(\mathrm{MAH})$, were optimized at the M062X/Def2-TZVP level of theory. The corresponding Gibbs free energies $\left(G^{\circ}\right.$ 's) are the sum of the thermal correction energy (with the scaling factor of 0.9708 applied) ( $\mathrm{E}_{\mathrm{G}_{-} \text {corr }}$ ) and the single point energy (at the M06-2X/Aug-cc-pVTZ level of theory) ( $\left.\mathrm{E}_{\mathrm{sp}}\right)$, i.e., $G^{\circ}=\mathrm{E}_{\mathrm{G}_{-} \text {corr }}+\mathrm{E}_{\mathrm{sp}}$. The procedure is similar to that described in the Computational Procedures in the main paper. The $\Delta G^{\circ} \mathrm{s}$ were calculated as follows,

$$
\Delta G^{\circ}=\left(G_{D o n^{+}}^{\circ}-G_{D o n-H}^{\circ}\right)-\left(G_{M A^{+}}^{\circ}-G_{\mathrm{MAH}}^{\circ}\right)
$$

Table S10. Thermal correction energy, single point energy and Gibbs free energy of the optimized structures

\begin{tabular}{|l|l|l|l|}
\hline Structures & $\mathbf{E}_{\mathbf{G} \text { _corr }}$ (Hartrees) & $\mathbf{E}_{\mathbf{s p}}$ (Hartrees) & $\boldsymbol{G}^{\circ}$ (Hartrees) \\
\hline BNAH & 0.199123 & -688.501204 & -688.302081 \\
\hline BNA $^{+}$ & 0.190215 & -687.690511 & -687.500296 \\
\hline DMPBIH & 0.22906 & -690.678634 & -690.449574 \\
\hline $\mathrm{DMPBI}^{+}$ & 0.221704 & -689.896608 & -689.674904 \\
\hline $\mathrm{HEH}(\mathrm{Me})$ & 0.201255 & -783.850391 & -783.649136 \\
\hline $\mathrm{HE}(\mathrm{Me})^{+}$ & 0.193087 & -783.033576 & -782.840489 \\
\hline $\mathrm{MAH}$ & 0.19194 & -596.041280 & -595.849340 \\
\hline $\mathrm{MA}^{+}$ & 0.182666 & -595.212739 & -595.030073 \\
\hline $\mathrm{TMBIH}^{\mathrm{TMBI}}{ }^{+}$ & 0.183856 & -498.949500 & -498.765644 \\
\hline
\end{tabular}

\begin{tabular}{llll}
\multicolumn{5}{l}{ Atomic coordinates of the optimized structures } \\
$\mathrm{BNA}^{+}$ & & \\
$\mathrm{C}$ & 2.980488989306 & 0.829657853068 & 0.619142506928 \\
$\mathrm{C}$ & 2.474999135978 & 2.030262564015 & 1.090246194510 \\
$\mathrm{H}$ & 4.027323192289 & 0.569726862120 & 0.718941035536 \\
$\mathrm{C}$ & 2.129154002521 & -0.072301187052 & -0.010315645884 \\
$\mathrm{C}$ & 1.140184536439 & 2.312989448742 & 0.900375851088 \\
$\mathrm{C}$ & 0.804528109877 & 0.267338681158 & -0.177898482568 \\
$\mathrm{C}$ & 2.728224846316 & -1.353989652274 & -0.548040860942 \\
$\mathrm{~N}$ & 0.334056920278 & 1.442256529983 & 0.272418108607 \\
$\mathrm{O}$ & 3.905922363997 & -1.383014371145 & -0.797643284727 \\
$\mathrm{H}$ & 3.101546435463 & 2.750245071365 & 1.596479276452 \\
$\mathrm{~N}$ & 1.871223813805 & -2.382189312207 & -0.747041551388 \\
$\mathrm{H}$ & 2.273182980287 & -3.250572375592 & -1.068173890029 \\
$\mathrm{H}$ & 0.975849962867 & -2.429935805356 & -0.292013767663 \\
$\mathrm{H}$ & 0.682733411989 & 3.234346071000 & 1.232882536056 \\
$\mathrm{H}$ & 0.087177075915 & -0.362724829515 & -0.686038376164 \\
$\mathrm{C}$ & -1.098851555723 & 1.826839981451 & 0.020517376124 \\
$\mathrm{C}$ & -2.021285714407 & 0.645596931879 & 0.019853536684 \\
$\mathrm{H}$ & -1.115769486964 & 2.346912321896 & -0.937179008007 \\
$\mathrm{H}$ & -1.355864455871 & 2.533750467766 & 0.807370455004 \\
$\mathrm{C}$ & -2.556404390407 & 0.175596889116 & -1.174268974621 \\
$\mathrm{C}$ & -2.332503557689 & 0.006397040478 & 1.218323224949 \\
$\mathrm{C}$ & -3.397315053488 & -0.930142611612 & -1.172493312469 \\
$\mathrm{H}$ & -2.327258437840 & 0.679363692613 & -2.106446655944 \\
$\mathrm{C}$ & -3.167535261738 & -1.099064037178 & 1.218296232961 \\
$\mathrm{H}$ & -1.931275849090 & 0.380732673601 & 2.153917317504
\end{tabular}




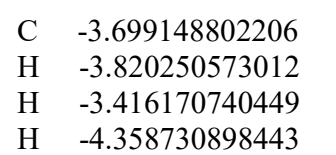

\section{BNAH}

H $\quad-1.787794497848$

C -1.186391637886

C -0.053810318595

H $\quad-1.884036946053$

C -0.650536334448

C $\quad 1.218899153741$

C 0.661648773972

C -1.645384257237

N 1.625275520293

O -2.753017542367

H $\quad-0.281822861550$

$\mathrm{N} \quad-1.261928408248$

$\mathrm{H} \quad-2.009402638101$

H $\quad-0.578327954699$

H 2.026493962842

H 1.043510866928

C $\quad 3.019773072867$

C 3.494855610716

H 3.619164588932

H $\quad 3.191606498150$

C 4.853929498499

C 2.611558005150

C $\quad 5.323211014278$

H 5.549291735511

C 3.082445185601

H 1.552846875579

C 4.435172578037

H 6.381674134237

$\mathrm{H} \quad 2.385154182151$

H 4.799151139546

\section{$\mathrm{DMBI}^{+}$}

C 2.894320956634

C 2.893689765642

C 1.723449858078

H 3.840882174604

C 1.722163658773

H $\quad 3.839781647630$

C 0.543323390083

H 1.726728993535

C 0.542697421696

H 1.724471819725

N $\quad-0.788151640592$

$\mathrm{N} \quad-0.789131832231$

C -1.541923076412

C $\quad-1.257819222938$

C -1.260038434146

H $\quad-2.343997871119$

H $\quad-0.872964864261$

H -0.909064627510

H -2.346220035330

H $\quad-0.911742191759$

H $\quad-0.875633883140$

H $\quad-2.620370486962$
$-1.567761828763$

$-1.286476418428$

$-1.587550157196$

$-2.425295493931$

0.021730652358

$-2.102048886597$

2.150874226740

0.024402165503

$-0.025380740598 \quad-1.068603252524$

$-0.559719608508 \quad-1.809649096527$

$0.294389614730 \quad-2.310554086961$

$\begin{array}{ll}-0.794950967847 & -2.621205118711\end{array}$

$-1.833641030411 \quad-1.201779834892$

$-0.085159759620 \quad-2.260624637630$

$-2.116288534299-1.176679793873$

$-2.718851660954 \quad-0.562017861970$

$-1.317842455501 \quad-1.740194795795$

$-2.313495038381 \quad-0.269841731712$

$1.261373641077 \quad-2.738082749890$

$-4.011639804467 \quad-0.278257721814$

$\begin{array}{ll}-4.600746513330 & 0.052994527198\end{array}$

$-4.455952157950 \quad-0.866158646206$

$0.539356177642 \quad-2.619500580065$

$\begin{array}{ll}-2.997130537526 & -0.673917487183\end{array}$

$-1.522738253463 \quad-1.421248937755$

$-0.836208741780 \quad-0.155180391134$

$-1.177662961961 \quad-2.267428849933$

$\begin{array}{ll}-2.598629400519 & -1.338381903439\end{array}$

$\begin{array}{ll}-0.848710756076 & 0.149771634607\end{array}$

$-0.208845279149 \quad 0.713732683680$

$-0.249214524545 \quad 1.306757688419$

$-1.333024298575 \quad-0.527548810594$

$0.394724346900 \quad 1.874440515411$

$-0.184181572864 \quad 0.486215980507$

$0.375580563809 \quad 2.174514845659$

$-0.266254584726 \quad 1.532366588410$

$0.881535040477 \quad 2.543842189536$

$0.846468798414 \quad 3.078214635180$

$0.701673229213 \quad-0.048439666392$ $-0.703969528490 \quad-0.048400739954$ $1.431580060320 \quad-0.024729322868$ $1.223882842898 \quad-0.067627952499$ $-1.432823277396 \quad-0.024650625947$ $-1.227030068580 \quad-0.067558974224$ $0.696875358513 \quad-0.000809383229$ $2.512883933917 \quad-0.024818549972$ $-0.697057788706 \quad-0.000770693888$ $-2.514129664811 \quad-0.024677785724$ $\begin{array}{lll}1.092149711201 & 0.026159548491\end{array}$ $\begin{array}{ll}-1.091134736936 & 0.026227841877\end{array}$ $0.000846177300 \quad 0.041457781980$ $2.471951813945 \quad 0.035646545890$ $\begin{array}{ll}-2.470513857301 & 0.035801526298\end{array}$ $2.475848931193 \quad 0.057646785650$ $2.977297669565 \quad 0.919289668576$ $2.977263227899 \quad-0.862876359267$

$-2.473434080953 \quad 0.057807346736$ $-2.976192944952 \quad-0.862692304149$ $-2.976151542881 \quad 0.919473525288$ $0.001331085043 \quad 0.063308747328$

DMBIH 


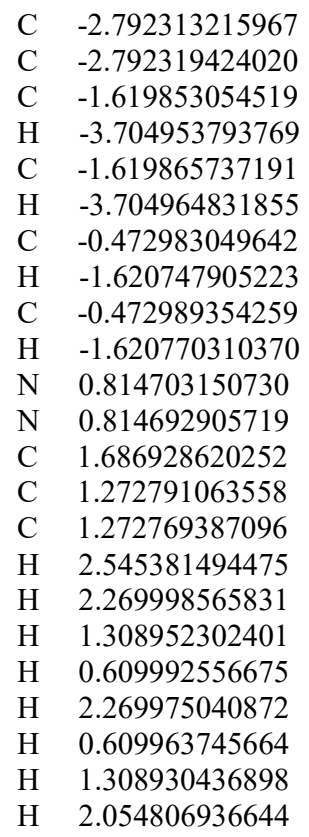

\section{$\mathrm{DMPBI}^{+}$}

$\begin{array}{llll}\mathrm{C} & -1.799982138166 & -2.458615732115 & -1.709990427408 \\ \mathrm{C} & -1.475938680357 & -3.402686528016 & -0.723241591469 \\ \mathrm{C} & -0.981808654151 & -1.379071085439 & -1.980991715282 \\ \mathrm{H} & -2.715758871045 & -2.580586626586 & -2.271682998048 \\ \mathrm{C} & -0.321389266720 & -3.302545216885 & 0.028591857421 \\ \mathrm{H} & -2.148887687052 & -4.230772746009 & -0.548783668574 \\ \mathrm{C} & 0.178591366934 & -1.279790900419 & -1.223197388254 \\ \mathrm{H} & -1.236936044833 & -0.650550447628 & -2.738125085299 \\ \mathrm{C} & 0.500513317438 & -2.216406973789 & -0.246032195327 \\ \mathrm{H} & -0.071932021529 & -4.034459195972 & 0.784340615493 \\ \mathrm{~N} & 1.207518489389 & -0.347651420930 & -1.232248655474 \\ \mathrm{~N} & 1.707463138135 & -1.811481738891 & 0.308005423653 \\ \mathrm{C} & 2.109715299724 & -0.689532324435 & -0.303143209309 \\ \mathrm{C} & 1.291048278772 & 0.759363774723 & -2.176075259963 \\ \mathrm{C} & 2.351472331432 & -2.483414167843 & 1.429178769720 \\ \mathrm{C} & 3.340179873140 & 0.046296565396 & -0.003223076628 \\ \mathrm{H} & 2.298084423401 & 1.164592468417 & -2.166317532625 \\ \mathrm{H} & 1.061668032192 & 0.382935824168 & -3.171066252891 \\ \mathrm{H} & 0.576699469385 & 1.535135316232 & -1.904370669396 \\ \mathrm{H} & 3.111183924118 & -1.832148069947 & 1.850031323322 \\ \mathrm{H} & 1.597083544176 & -2.695249334802 & 2.184390057140 \\ \mathrm{H} & 2.805798426950 & -3.414682353686 & 1.094116173419 \\ \mathrm{C} & 3.276361775854 & 1.400281296539 & 0.324727712320 \\ \mathrm{C} & 4.569553018851 & -0.610678682395 & -0.047082716832 \\ \mathrm{C} & 4.442358287107 & 2.091467307831 & 0.610804708179 \\ \mathrm{H} & 2.318220629674 & 1.903250496766 & 0.373662493200 \\ \mathrm{C} & 5.731060442261 & 0.089991980233 & 0.234148431580 \\ \mathrm{H} & 4.616718629093 & -1.658415902679 & -0.318061448409 \\ \mathrm{C} & 5.666875159357 & 1.437671353756 & 0.563886805599 \\ \mathrm{H} & 4.394749570765 & 3.139091910411 & 0.874830900282 \\ \mathrm{H} & 6.686529819955 & -0.414730463972 & 0.191403916826 \\ \mathrm{H} & 6.57568115751 & 1.981148617964 & 0.785400703034 \\ & & & \\ \mathrm{DMPBIH} & & & \\ \mathrm{C} & -0.566222483953 & -2.935709048965 & -2.275984710869 \\ \mathrm{C} & -0.301096853739 & -3.767951624583 & -1.209072645254 \\ & & & \end{array}$

$\begin{array}{ll}-0.690914503962 & 0.245939002543\end{array}$ $\begin{array}{lll}0.690891474978 & 0.245940100695\end{array}$ $-1.411514129776 \quad-0.019854826175$ $\begin{array}{ll}-1.228568215685 & 0.465553220881\end{array}$ $1.411502059269-0.019852580236$ $1.228536636980 \quad 0.465555177873$ $-2.493369669226 \quad-0.002620358583$ $0.701679011185 \quad-0.295991564543$ $\begin{array}{ll}2.493357563121 & -0.002616391743\end{array}$ $-1.140213425027 \quad-0.622740655834$ $1.140224189478 \quad-0.622738846620$ $0.000009128917-0.382879521980$ $-2.412718368765 \quad-0.122935366464$ $2.412732457053 \quad-0.122931543235$ $0.000013503406-1.057440363385$ $-2.616850980029-0.512716044276$ $\begin{array}{ll}-2.437492658029 & 0.975662315709\end{array}$ $-3.204455757464 \quad-0.468768478800$ $2.616874663186 \quad-0.512711925923$ $3.204464433357 \quad-0.468763372170$ $\begin{array}{ll}2.437505319562 & 0.975666177336\end{array}$ $0.000009950266 \quad 0.667672205233$ $\begin{array}{ll}-0.701680336412 & -0.295992680305\end{array}$ 


$\begin{array}{llll}\mathrm{C} & -0.299243031057 & -1.557101829006 & -2.201740587563 \\ \mathrm{H} & -0.974078641824 & -3.345418996038 & -3.190298405897 \\ \mathrm{C} & 0.244139033554 & -3.262491600747 & -0.015499791824 \\ \mathrm{H} & -0.501799066869 & -4.827843298273 & -1.289913198849 \\ \mathrm{C} & 0.214846374882 & -1.062781484484 & -1.026770543682 \\ \mathrm{H} & -0.487041803104 & -0.910370495673 & -3.048601211387 \\ \mathrm{C} & 0.485345430697 & -1.911454518163 & 0.060989046278 \\ \mathrm{H} & 0.472662504961 & -3.921468830760 & 0.811710399907 \\ \mathrm{~N} & 0.558055099928 & 0.233949253566 & -0.656387364887 \\ \mathrm{~N} & 0.995990494736 & -1.134461469780 & 1.096022648235 \\ \mathrm{C} & 1.398441576823 & 0.157011312513 & 0.539291716495 \\ \mathrm{C} & 0.836303189925 & 1.237899320198 & -1.651295800915 \\ \mathrm{C} & 1.779910995139 & -1.726827414191 & 2.149355715834 \\ \mathrm{H} & 1.134057942374 & 0.963204650139 & 1.234144655558 \\ \mathrm{C} & 2.889403202959 & 0.260067440102 & 0.245024724664 \\ \mathrm{H} & 1.030429719432 & 2.189898682161 & -1.156928763161 \\ \mathrm{H} & 1.702661932683 & 0.987513180591 & -2.276722890287 \\ \mathrm{H} & -0.036570111534 & 1.361732133957 & -2.292180853417 \\ \mathrm{H} & 2.039258532266 & -0.959754554330 & 2.879175529609 \\ \mathrm{H} & 1.186877534833 & -2.488457513483 & 2.655655502919 \\ \mathrm{H} & 2.706985987430 & -2.185548754642 & 1.782576504141 \\ \mathrm{C} & 3.641827448417 & 1.279520304666 & 0.810284659694 \\ \mathrm{C} & 3.514257030783 & -0.661078815828 & -0.596233763817 \\ \mathrm{C} & 5.002665832930 & 1.388219613715 & 0.545570085144 \\ \mathrm{H} & 3.158175645206 & 1.996589962546 & 1.464921667145 \\ \mathrm{C} & 4.869934219011 & -0.555155385376 & -0.861347788980 \\ \mathrm{H} & 2.934049000734 & -1.461135820417 & -1.042398531808 \\ \mathrm{C} & 5.617308635339 & 0.470387320097 & -0.290684466237 \\ \mathrm{H} & 5.578921017530 & 2.187906454302 & 0.992756590979 \\ \mathrm{H} & 5.348311961930 & -1.273378153560 & -1.514694851435 \\ \mathrm{H} & 6.676174647576 & 0.550037979746 & -0.499877276334\end{array}$

$\begin{array}{llll}\mathrm{HEH}(\mathrm{Me}) & & \\ \mathrm{H} & 0.786761489876 & -1.424196043546 & -0.907631858180 \\ \mathrm{C} & 0.907747949877 & -1.649789137951 & 0.155362502632 \\ \mathrm{C} & 2.050985303341 & -0.851925703590 & 0.735769587796 \\ \mathrm{H} & 1.138286813504 & -2.718330883949 & 0.178963201801 \\ \mathrm{C} & -0.398199592936 & -1.399606434272 & 0.875820183609 \\ \mathrm{C} & 1.886272443521 & -0.018568102540 & 1.781524979263 \\ \mathrm{C} & 3.379934557249 & -0.998956591459 & 0.130778451439 \\ \mathrm{C} & -0.494870635792 & -0.550658760602 & 1.918134301292 \\ \mathrm{C} & -1.517279084994 & -2.169945346660 & 0.320393641383 \\ \mathrm{~N} & 0.635165362850 & 0.117815454207 & 2.348648490066 \\ \mathrm{C} & 2.951027290974 & 0.815473832612 & 2.430391221778 \\ \mathrm{O} & 4.400123656272 & -0.441404367620 & 0.464791461187 \\ \mathrm{O} & 3.350070070568 & -1.869482820054 & -0.896321313012 \\ \mathrm{C} & -1.724578352157 & -0.215304748150 & 2.715351110372 \\ \mathrm{O} & -1.389405652138 & -2.921026265268 & -0.615519644650 \\ \mathrm{O} & -2.710103560146 & -1.986669064770 & 0.924579263591 \\ \mathrm{H} & 0.541672995262 & 0.745827129529 & 3.126873494393 \\ \mathrm{H} & 3.756936272475 & 0.188220855873 & 2.805762632053 \\ \mathrm{H} & 3.402274232972 & 1.493959287506 & 1.709368962826 \\ \mathrm{H} & 2.532273436440 & 1.393715566018 & 3.254498676140 \\ \mathrm{C} & 4.595434634912 & -2.080126078872 & -1.550036241717 \\ \mathrm{H} & -2.141462455317 & -1.105941904685 & 3.179631431554 \\ \mathrm{H} & -1.483440624260 & 0.505761596177 & 3.496157858371 \\ \mathrm{H} & -2.497925272731 & 0.206498835211 & 2.077696937958 \\ \mathrm{C} & -3.782468154330 & -2.742653263363 & 0.371101209673 \\ \mathrm{H} & 4.971195069251 & -1.145465215444 & -1.964607937092 \\ \mathrm{H} & 5.331751901872 & -2.473029057451 & -0.849949554623 \\ \mathrm{H} & 4.398451305671 & -2.797710247221 & -2.340963540494\end{array}$




\begin{tabular}{|c|c|c|c|}
\hline $\mathrm{H}$ & -4.660140611886 & -2.487899516123 & \\
\hline $\mathrm{H}$ & -3.932830432161 & -2.481855514891 & -0.675564464524 \\
\hline $\mathrm{H}$ & -3.572309358039 & -3.809133488652 & 0.438823125274 \\
\hline \multicolumn{4}{|c|}{$\mathrm{HE}(\mathrm{Me})^{+}$} \\
\hline $\mathrm{C}$ & 0.149350303064 & -0.563662123166 & 0.016887255191 \\
\hline $\mathrm{C}$ & 1.294931766857 & 0.217963053352 & -0.001983159078 \\
\hline $\mathrm{H}$ & 0.234589092711 & -1.643331839224 & 0.029734259769 \\
\hline $\mathrm{C}$ & -1.127955593855 & -0.012027443688 & 0.020104010849 \\
\hline $\mathrm{C}$ & 1.156710025990 & 1.601429296316 & -0.018215443296 \\
\hline $\mathrm{C}$ & 2.662284206193 & -0.406037576958 & -0.005003997439 \\
\hline $\mathrm{C}$ & -1.257004118696 & 1.368919864366 & 0.003832951020 \\
\hline $\mathrm{C}$ & -2.268696901173 & -0.997275514011 & 0.041486020771 \\
\hline $\mathrm{N}$ & -0.105349954703 & 2.080202875298 & -0.014205751683 \\
\hline $\mathrm{C}$ & 2.254728461428 & 2.607880622966 & -0.039526495561 \\
\hline $\mathrm{O}$ & 3.674937066636 & 0.235569281015 & -0.021063155749 \\
\hline $\mathrm{O}$ & 2.590822062183 & -1.723707352346 & 0.012064700599 \\
\hline $\mathrm{C}$ & -2.519003301118 & 2.165701318302 & 0.003860165382 \\
\hline $\mathrm{O}$ & -2.070907819259 & -2.176389118638 & 0.054692614892 \\
\hline $\mathrm{O}$ & -3.459884668719 & -0.421423495580 & 0.043707300084 \\
\hline $\mathrm{H}$ & -0.20037768 & 3.09 & 7119 \\
\hline $\mathrm{H}$ & 2.900667726832 & 2.475298058376 & 0.827564377124 \\
\hline $\mathrm{H}$ & 2.886889252944 & 2.453473657865 & -0.913135222576 \\
\hline $\mathrm{H}$ & 1.854993163402 & 3.620873165177 & -0.049061846129 \\
\hline $\mathrm{C}$ & 3.853157486054 & $-2.416444 C$ & 0.010 \\
\hline $\mathrm{H}$ & -3.111158580710 & 1.93 & 0.88 \\
\hline $\mathrm{H}$ & -2.305126408687 & 3.233211711525 & 202914455 \\
\hline $\mathrm{H}$ & -3.125032724130 & 1.909034149287 & -0.863339314464 \\
\hline $\mathrm{C}$ & -4.580179392751 & -1.329222851468 & 0.063949516561 \\
\hline $\mathrm{H}$ & 4.411060762869 & -2.157824521110 & 142127912 \\
\hline $\mathrm{H}$ & 4.425 & 6046 & 0.89 \\
\hline $\mathrm{H}$ & 3.6046163 & -3.471459 & 0.0258 \\
\hline $\mathrm{H}$ & -5.463919587888 & -0.700991236868 & 0.063035171901 \\
\hline $\mathrm{H}$ & -4.550378393795 & -1.965596963695 & -0.817432399185 \\
\hline $\mathrm{H}$ & -4.536346210138 & -1.943267384514 & 0.960455405164 \\
\hline \multicolumn{4}{|c|}{$\mathrm{MA}^{+}$} \\
\hline $\mathrm{H}$ & -1.138558187314 & 0.318668030089 & -0.054054446869 \\
\hline $\mathrm{C}$ & -0.153529571574 & 0.767208207682 & -0.037508222627 \\
\hline $\mathrm{C}$ & 0.923787600260 & 0.002213475053 & 3612002893 \\
\hline $\mathrm{C}$ & 0.054581522179 & 2.029088071095 & -0.494586160351 \\
\hline $\mathrm{C}$ & 2.237087322407 & 0.553867333364 & 0.512776999958 \\
\hline $\mathrm{C}$ & 0.725617355269 & -1.276118741351 & 0.991083934228 \\
\hline $\mathrm{C}$ & 1.354315440905 & 2.582727382013 & -0.430718183802 \\
\hline $\mathrm{H}$ & -0.761109779311 & 2.616731433366 & -0.891400034394 \\
\hline $\mathrm{C}$ & 2.421749118032 & 1.877418046639 & 0.052026802088 \\
\hline $\mathrm{N}$ & 3.273073293372 & -0.203091708730 & 0.987803789496 \\
\hline $\mathrm{C}$ & 1.768004006839 & -1.998768759084 & 1.554191546705 \\
\hline $\mathrm{H}$ & 1.511275594276 & 3.599684120607 & -0.766125465072 \\
\hline $\mathrm{H}$ & 3.383234983239 & 2.361282858069 & 0.107042617666 \\
\hline $\mathrm{C}$ & 3.072472922884 & -1.426183528260 & 1.567208419889 \\
\hline $\mathrm{C}$ & 4.645683387954 & 0.309143910417 & 0.862227316248 \\
\hline $\mathrm{C}$ & 1.556485254938 & -3.285912466141 & 2.120894792660 \\
\hline $\mathrm{C}$ & 4.117920597535 & -2.142890068858 & 2.192946733211 \\
\hline $\mathrm{H}$ & 5.326432065643 & -0.524220749316 & 0.739134475523 \\
\hline $\mathrm{H}$ & 4.717349559379 & 0.919430030910 & -0.029671037524 \\
\hline $\mathrm{H}$ & 4.920204045319 & 0.892769915320 & 1.740666026051 \\
\hline $\mathrm{C}$ & 2.582936880020 & -3.963684875722 & 2.696735762833 \\
\hline $\mathrm{H}$ & 0.557517430678 & -3.701413723049 & 2.086755743042 \\
\hline $\mathrm{C}$ & 3.866085357070 & -3.370733399593 & 2.739662454709 \\
\hline $\mathrm{H}$ & 5.107648533881 & -1.725964201153 & 2.283601841710 \\
\hline $\mathrm{H}$ & 2.427176927978 & -4.940228732529 & 3.132900039896 \\
\hline
\end{tabular}




\begin{tabular}{lllc}
$\mathrm{H}$ & 4.675040722611 & -3.899152604817 & 3.227207146798 \\
$\mathrm{H}$ & -0.267700344467 & -1.710804116021 & 0.961784105033 \\
\multicolumn{1}{l}{$\mathrm{MAH}$} & & \\
$\mathrm{H}$ & -0.320707027352 & 0.016574462707 & 0.012451951794 \\
$\mathrm{H}$ & 0.110920265394 & -1.548848284080 & -0.668347567128 \\
$\mathrm{C}$ & -0.079897469544 & -1.023742651705 & 0.267768039415 \\
$\mathrm{C}$ & 1.137286221431 & -1.030384853221 & 1.149010261598 \\
$\mathrm{C}$ & -1.252030190193 & -1.621594839395 & 0.993609377655 \\
$\mathrm{C}$ & 2.416493624078 & -1.243705327608 & 0.666566256547 \\
$\mathrm{C}$ & 0.948052579488 & -0.759929010018 & 2.511364514745 \\
$\mathrm{C}$ & -1.377992125827 & -1.335483703570 & 2.360080810792 \\
$\mathrm{C}$ & -2.216081873102 & -2.389977919891 & 0.365264883595 \\
$\mathrm{H}$ & 2.547439167538 & -1.460277446753 & -0.387424846961 \\
$\mathrm{C}$ & 3.523121148622 & -1.164008415285 & 1.503505627909 \\
$\mathrm{C}$ & 2.056245254225 & -0.686407172314 & 3.354694261609 \\
$\mathrm{~N}$ & -0.364697929104 & -0.585422269276 & 2.979086681059 \\
$\mathrm{C}$ & -2.487132852524 & -1.810611480433 & 3.059193944710 \\
$\mathrm{C}$ & -3.325938504658 & -2.858722558125 & 1.058045244313 \\
$\mathrm{H}$ & -2.101669509575 & -2.610638093912 & -0.689802198663 \\
$\mathrm{C}$ & 3.333781088642 & -0.878163361297 & 2.845046686418 \\
$\mathrm{H}$ & 4.518009592777 & -1.322567545139 & 1.110219397029 \\
$\mathrm{H}$ & 1.932252021390 & -0.502225143170 & 4.412066179613 \\
$\mathrm{C}$ & -0.565470426003 & -0.130368742197 & 4.334742521140 \\
$\mathrm{C}$ & -3.456414852165 & -2.558312604410 & 2.403413491228 \\
$\mathrm{H}$ & -2.593016066955 & -1.621948905227 & 4.117744003527 \\
$\mathrm{H}$ & -4.076924539438 & -3.449274842267 & 0.551206189570 \\
$\mathrm{H}$ & 4.182976288670 & -0.817363520164 & 3.513364345569 \\
$\mathrm{H}$ & -1.576642129961 & 0.259121755575 & 4.433543808420 \\
$\mathrm{H}$ & -0.417962853692 & -0.922363454501 & 5.079654680696 \\
$\mathrm{H}$ & 0.128501106674 & 0.681021371706 & 4.544458191160 \\
$\mathrm{H}$ & -4.311103648837 & -2.919115446029 & 2.960913262642 \\
& & &
\end{tabular}

$\mathrm{TMBI}^{+}$

$\begin{array}{llll}\mathrm{C} & 2.879707870957 & 0.701520562072 & 0.014074928912 \\ \mathrm{C} & 2.879593852487 & -0.701751865483 & 0.014483793767 \\ \mathrm{C} & 1.705587790411 & 1.429681558929 & 0.009067485300 \\ \mathrm{H} & 3.825301880392 & 1.225748521687 & 0.015872493038 \\ \mathrm{C} & 1.705347327539 & -1.429711234107 & 0.009877724748 \\ \mathrm{H} & 3.825102793885 & -1.226133318084 & 0.016603073211 \\ \mathrm{C} & 0.526643350646 & 0.695188007167 & 0.005183961757 \\ \mathrm{H} & 1.709986512093 & 2.510891579583 & 0.002940710121 \\ \mathrm{C} & 0.526551315790 & -0.694997786626 & 0.005546865989 \\ \mathrm{H} & 1.709494578810 & -2.510929030915 & 0.004418383830 \\ \mathrm{~N} & -0.806541341946 & 1.089434034598 & 0.000015294004 \\ \mathrm{~N} & -0.806649326968 & -1.088925584307 & 0.000499799831 \\ \mathrm{C} & -1.580755889028 & 0.000283549921 & -0.014153620846 \\ \mathrm{C} & -1.235617800369 & 2.481609486762 & 0.009884243644 \\ \mathrm{C} & -1.236807699097 & -2.480714723298 & 0.011201273651 \\ \mathrm{H} & -2.288333767642 & 2.542921941632 & 0.264810592690 \\ \mathrm{H} & -0.662844600132 & 3.014736180310 & 0.765894262362 \\ \mathrm{H} & -1.062172717986 & 2.930960344571 & -0.966788113199 \\ \mathrm{H} & -2.290284881857 & -2.540587511148 & 0.263483308264 \\ \mathrm{H} & -1.061460343442 & -2.931435279073 & -0.964508095965 \\ \mathrm{H} & -0.666363244956 & -3.013484821013 & 0.769238029995 \\ \mathrm{C} & -3.064139242184 & -0.000698551150 & -0.036140331492 \\ \mathrm{H} & -3.439331576556 & 0.882501462257 & -0.546507728441 \\ \mathrm{H} & -3.437190997585 & -0.874839845269 & -0.563700240009 \\ \mathrm{H} & -3.462113843260 & -0.011217679015 & 0.980790904838\end{array}$

TMBIH 


$\begin{array}{llcc}\mathrm{C} & 0.724635668459 & 3.435222876656 & -1.122381886013 \\ \mathrm{C} & 1.990886893834 & 2.968227024494 & -1.409387622846 \\ \mathrm{C} & -0.085195680990 & 2.790849219480 & -0.172570252354 \\ \mathrm{H} & 0.349977105587 & 4.317276881747 & -1.624239411741 \\ \mathrm{C} & 2.507440290062 & 1.834679779131 & -0.760211675059 \\ \mathrm{H} & 2.603625396412 & 3.486127420581 & -2.135045958761 \\ \mathrm{C} & 0.415172642631 & 1.669452256099 & 0.446891152059 \\ \mathrm{H} & -1.070453959368 & 3.170904898966 & 0.063669039737 \\ \mathrm{C} & 1.704888865547 & 1.193802618874 & 0.154566896229 \\ \mathrm{H} & 3.508000732094 & 1.482362424055 & -0.974072623073 \\ \mathrm{~N} & -0.152338912165 & 0.816250140604 & 1.390386823415 \\ \mathrm{~N} & 1.923471541009 & 0.050689859211 & 0.919891663984 \\ \mathrm{C} & 0.948290954416 & 0.076301435803 & 2.013047954059 \\ \mathrm{C} & -1.249074477133 & 1.270975983053 & 2.204899933370 \\ \mathrm{C} & 3.267479294228 & -0.394734301546 & 1.181191107889 \\ \mathrm{H} & 0.624992880243 & -0.943503747731 & 2.246046351080 \\ \mathrm{H} & -1.574261944717 & 0.461801273328 & 2.859289485275 \\ \mathrm{H} & -0.994919217745 & 2.144353820753 & 2.820189074987 \\ \mathrm{H} & -2.088332893900 & 1.537466400222 & 1.562269510040 \\ \mathrm{H} & 3.237199866967 & -1.312671070850 & 1.768738203872 \\ \mathrm{H} & 3.762043004718 & -0.620161508714 & 0.236238829817 \\ \mathrm{H} & 3.870987920829 & 0.349801932301 & 1.717294987318 \\ \mathrm{C} & 1.483846119157 & 0.752024102970 & 3.276455986040 \\ \mathrm{H} & 0.713207122359 & 0.790443085168 & 4.046928620747 \\ \mathrm{H} & 2.329240420083 & 0.194431315519 & 3.680666508321 \\ \mathrm{H} & 1.808228367382 & 1.770381879822 & 3.050551301606\end{array}$

\section{Synthesis of 1,2,3-trimethylbenzimidazoline (TMBIH) and 1,2,3-trimethyl-2-deutero-benzimidazoline (TMBIH-2-d)}

The syntheses used the same standard procedures to make the 1,3-dimehtyl-2-phenylbenzimidazoline (DMPBIH and DMPBIH-2d). ${ }^{3}$

$5.028 \mathrm{~g}$ of 2-methylbenzimidazole $(38.04 \mathrm{mmol})$ was added to a $75 \mathrm{~mL}$ pressure reaction vessel. A slurry was made by adding 25 $\mathrm{mL}$ of methanol. $9.47 \mathrm{~mL}$ of iodomethane $(152.12 \mathrm{mmol})$ and $1.520 \mathrm{~g}$ of sodium hydroxide $(38.00 \mathrm{mmol})$ were then added. The pressure reaction vessel was placed into a $120^{\circ} \mathrm{C}$ hot oil bath and allowed to sit for 12 hours. It was then allowed to cool to room temperature overnight and the solid product was vacuum filtered. It was recrystallized three times using ethanol. The slightly orange crystals of 1,2,3-trimethylbenzimidazolium iodide ( $\left.\mathrm{TMBI}^{+} \mathrm{I}^{-}\right)$were vacuum filtered and dried. The yield was about 80\%. M.p. 252.8 $-255.4{ }^{\circ} \mathrm{C} ;{ }^{1} \mathrm{H}$ NMR for $\mathrm{TMBI}^{+}, \delta\left(\mathrm{ppm}, \mathrm{D}_{2} \mathrm{O}\right): 7.66(2 \mathrm{H}, \mathrm{s}), 7.53(2 \mathrm{H}, \mathrm{s}), 3.68\left(6 \mathrm{H}\right.$ in $\left.2 \mathrm{CH}_{3}, \mathrm{~s}\right), 2.75\left(3 \mathrm{H}\right.$ in $\left.\mathrm{CH}_{3}, \mathrm{~s}\right)$.

$2.000 \mathrm{~g}$ of $\mathrm{TMBI}^{+} \mathrm{I}^{-}(6.94 \mathrm{mmol})$ was added to a $50 \mathrm{~mL}$ round bottom flask. $20 \mathrm{~mL}$ of methanol was added to form a slurry. The flask was placed into a $0^{\circ} \mathrm{C}$ ice bath and allowed to stir thoroughly. $0.389 \mathrm{~g}$ of sodium borohydride $(10.04$ mmol) was then slowly added and the reaction was allowed to stir on ice for 30 minutes and then at room temperature for another 30 minutes. Water was added and the crude product was extracted three times using anhydrous diethyl ether. The ethereal extraction solution was dried over magnesium sulfate. The ether was rotary-evaporated. The dry colorless sticky product was purified via column chromatography (silica gel) using hexanes containing $2 \%$ ethyl acetate as the eluent. The product was stored in the freezer at $-20^{\circ} \mathrm{C}$ in order to solidify and form large white pieces of solid of TMBIH. The yield was about $80 \%$. Note that the NMRs of the product before or after the column chromatography purification are the same and both batches of the products gave the same kinetic results. The TMBIH-2-d was synthesized using the same procedures except that the $\mathrm{NaBD}_{4}$ and methanol- $\mathrm{d}_{4}$ were used and no column purification was performed. (Note that in the DMPBIH-2-d synthesis, the reduction by $\mathrm{NaBD}_{4}$ was carried out in anhydrous acetonitrile.) ${ }^{1} \mathrm{H}$ NMR for TMBIH, $\delta\left(\mathrm{ppm}, \mathrm{CD}_{3} \mathrm{CN}\right)$ : $6.61-6.63(2 \mathrm{H}, \mathrm{m}), 6.39-6.41(2 \mathrm{H}, \mathrm{m}), 4.03(1 \mathrm{H}, \mathrm{d}), 1.43-1.45(3 \mathrm{H}, \mathrm{d})$, $2.65(6 \mathrm{H}, \mathrm{s})$. No apparent NMR peak for 2-H of the TMBIH-2-d was observed. 


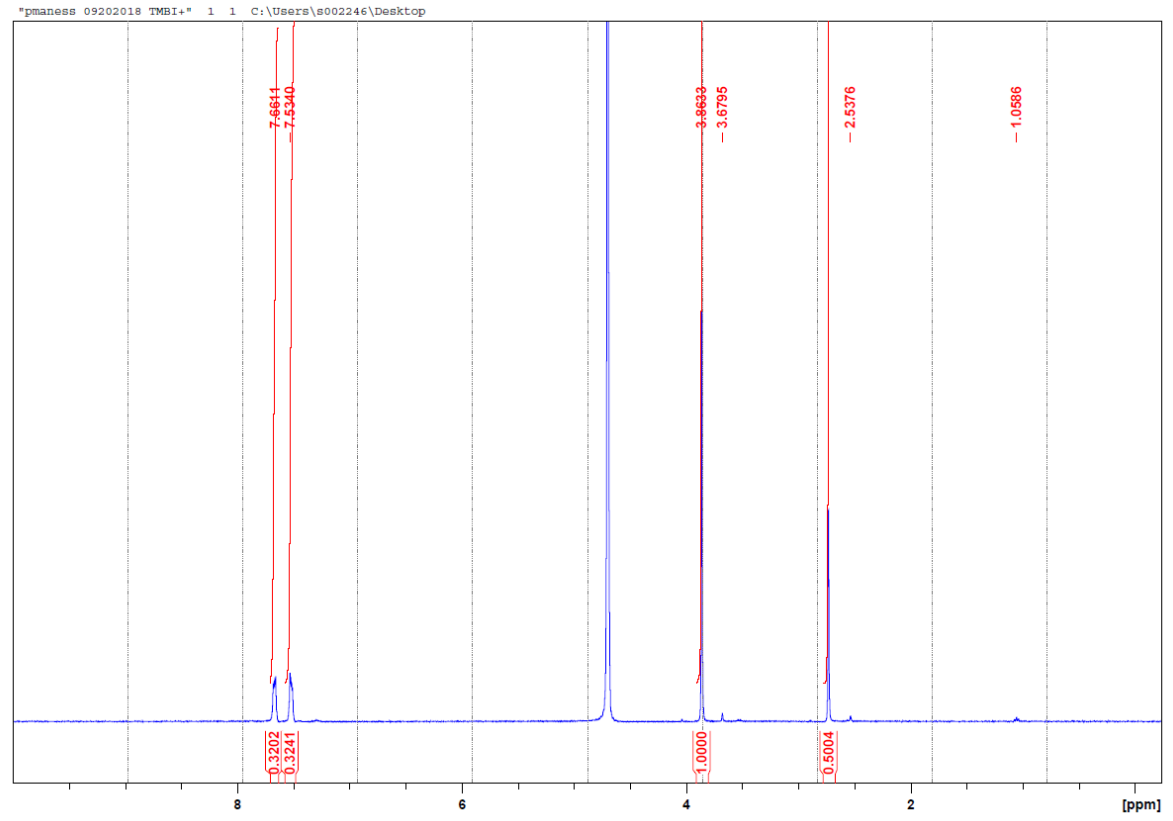

The ${ }^{1} \mathrm{H}$ NMR of $\mathrm{TMBI}^{+} \mathrm{I}^{-}$in $\mathrm{D}_{2} \mathrm{O}$

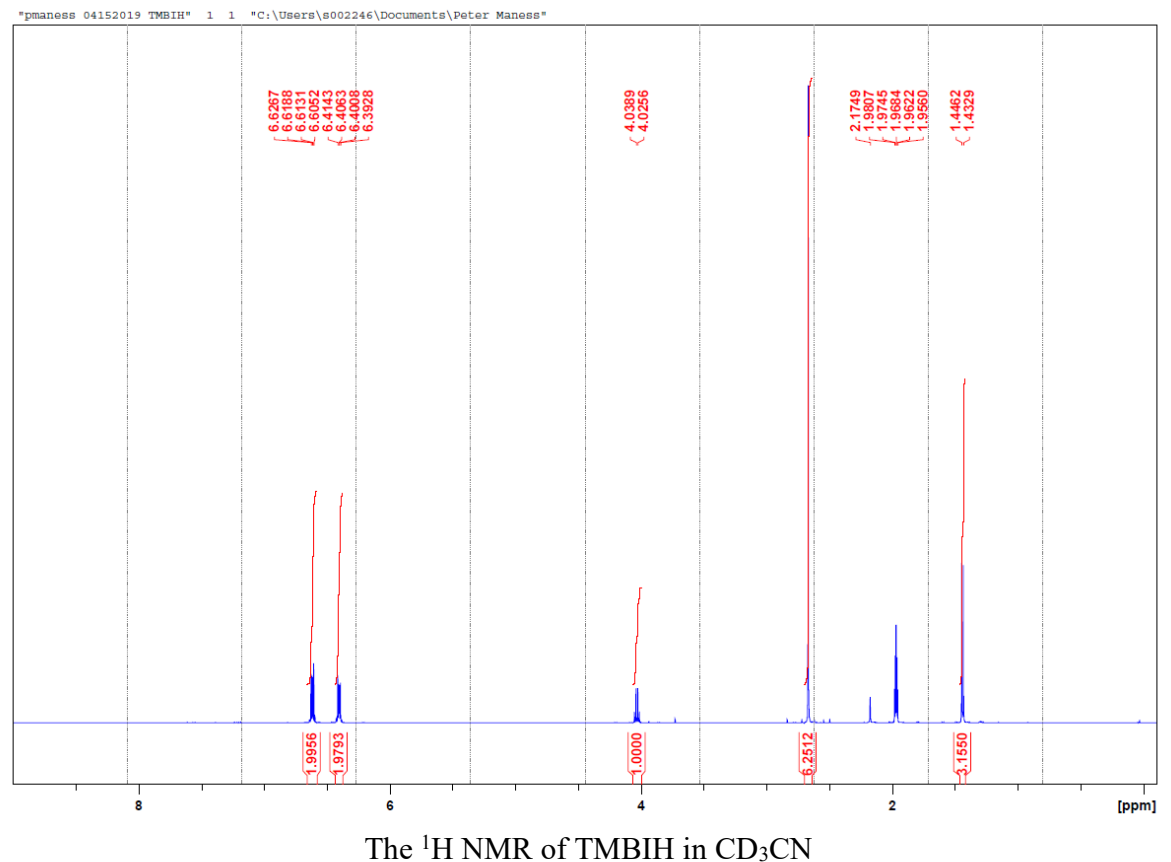




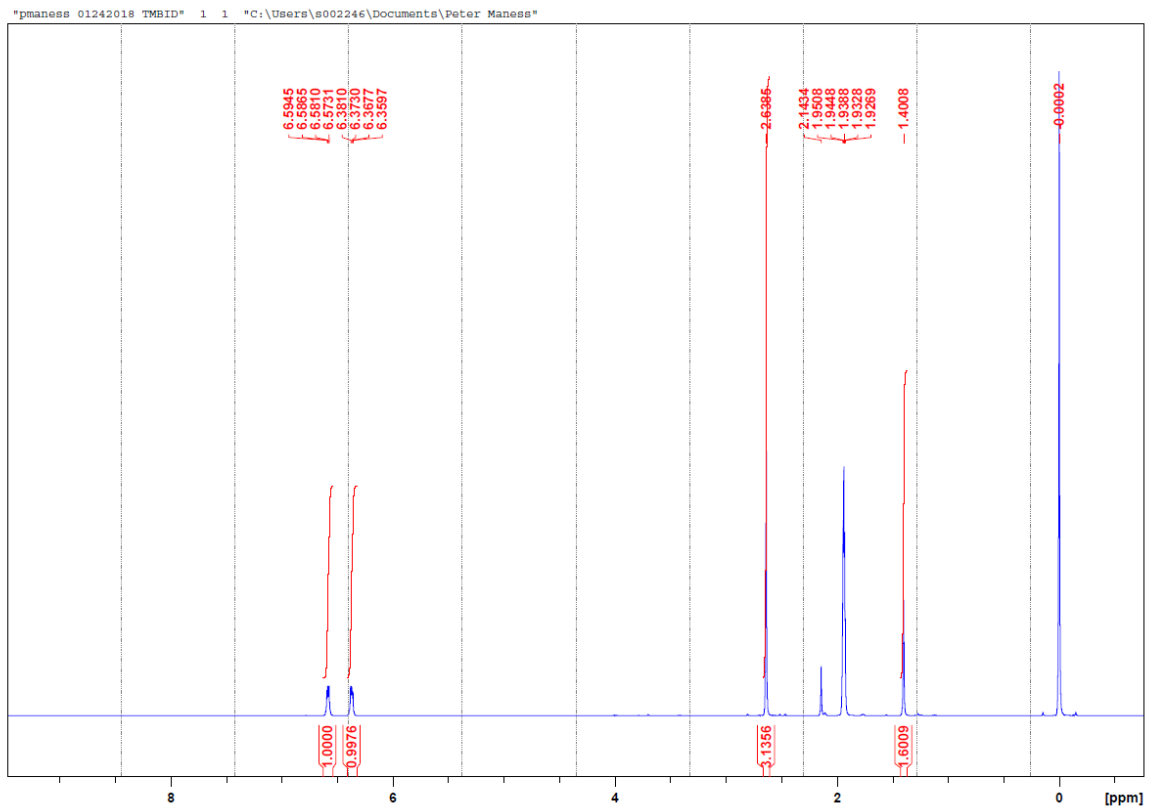

The ${ }^{1} \mathrm{H}$ NMR of TMBIH-2-d in $\mathrm{CD}_{3} \mathrm{CN}$

${ }^{1}$ H NMRs for HEH, HEH-4,4-d,d, DMPBIH, DMPBIH-2-d

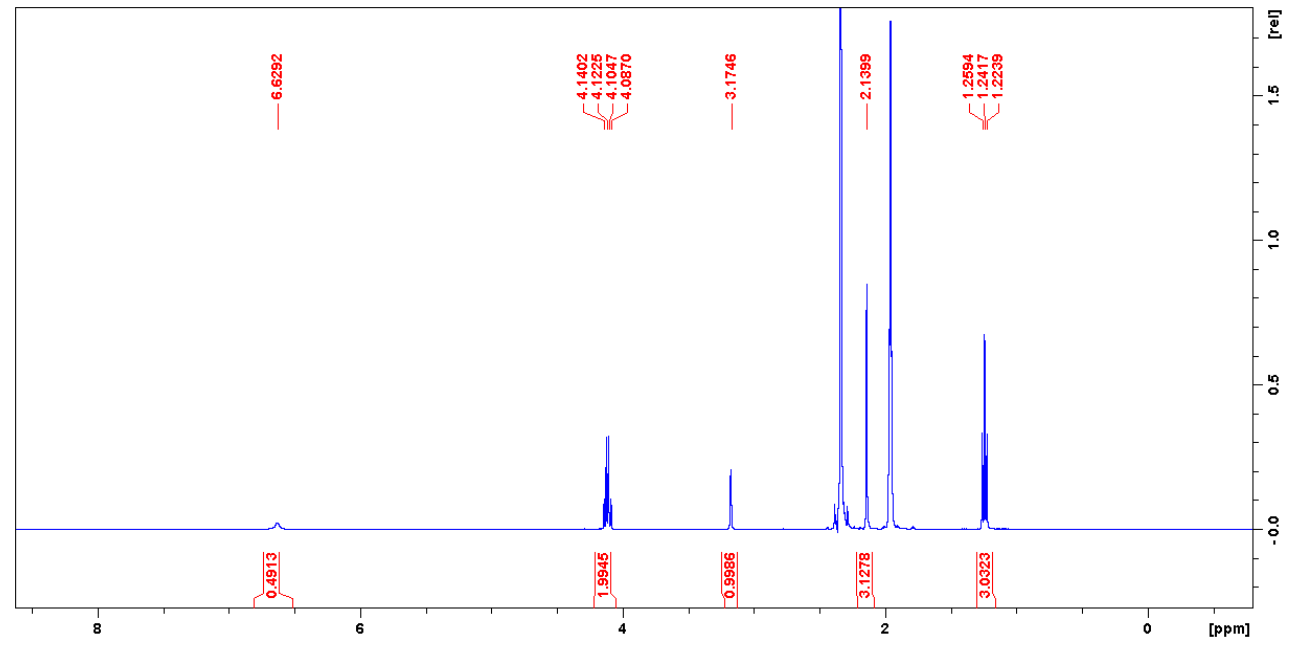

The ${ }^{1} \mathrm{H}$ NMR of $\mathrm{HEH}$ in $\mathrm{CD}_{3} \mathrm{CN}$ 

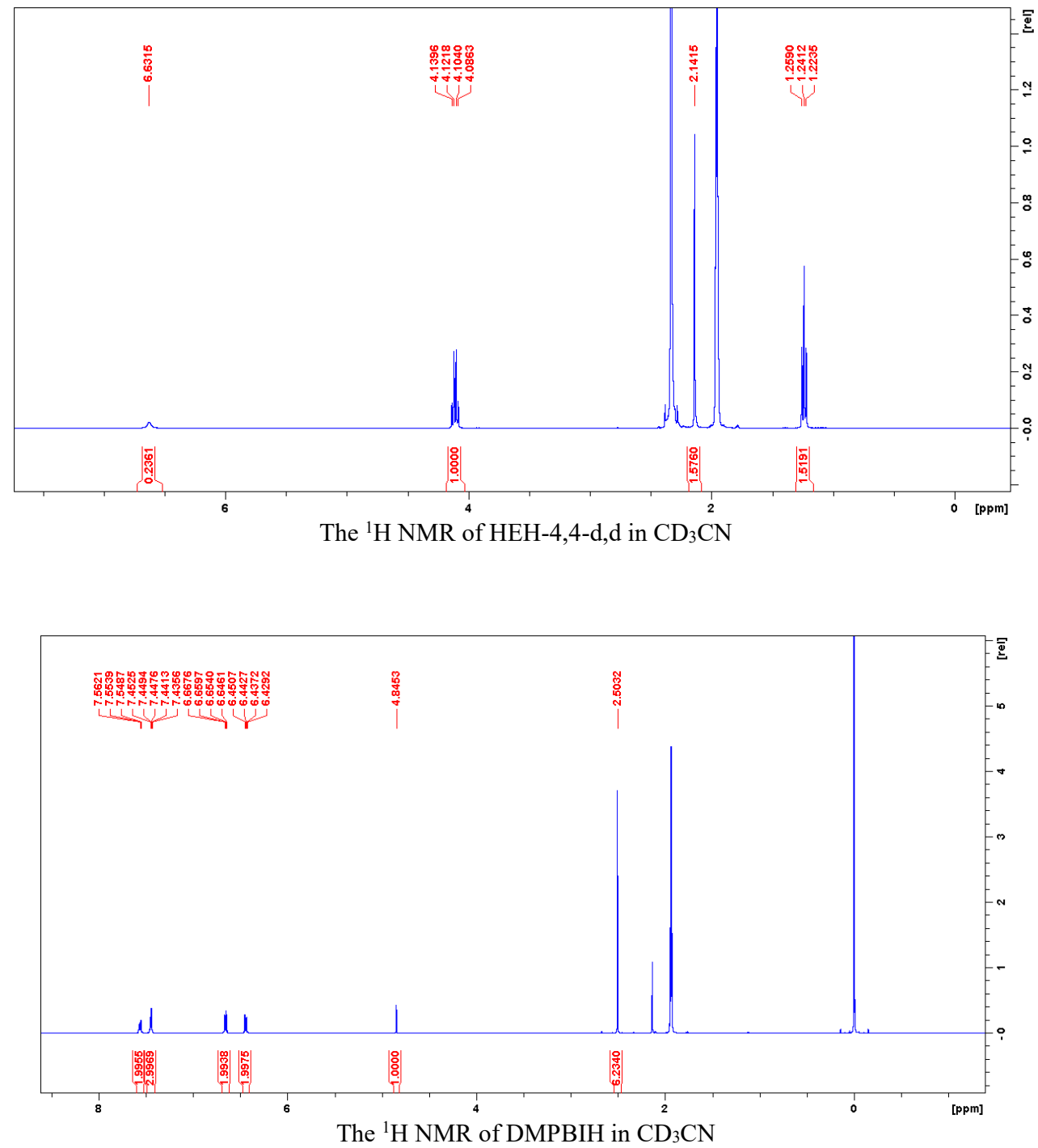

2018-12-20 DMPBID Proton NMR
Reduction in Acetonitrile Test for H content
Second Recrystalization

The ${ }^{1} \mathrm{H}$ NMR of $\mathrm{HEH}-4,4-\mathrm{d}$,d in $\mathrm{CD}_{3} \mathrm{CN}$

The ${ }^{1} \mathrm{H}$ NMR of DMPBIH in $\mathrm{CD}_{3} \mathrm{CN}$

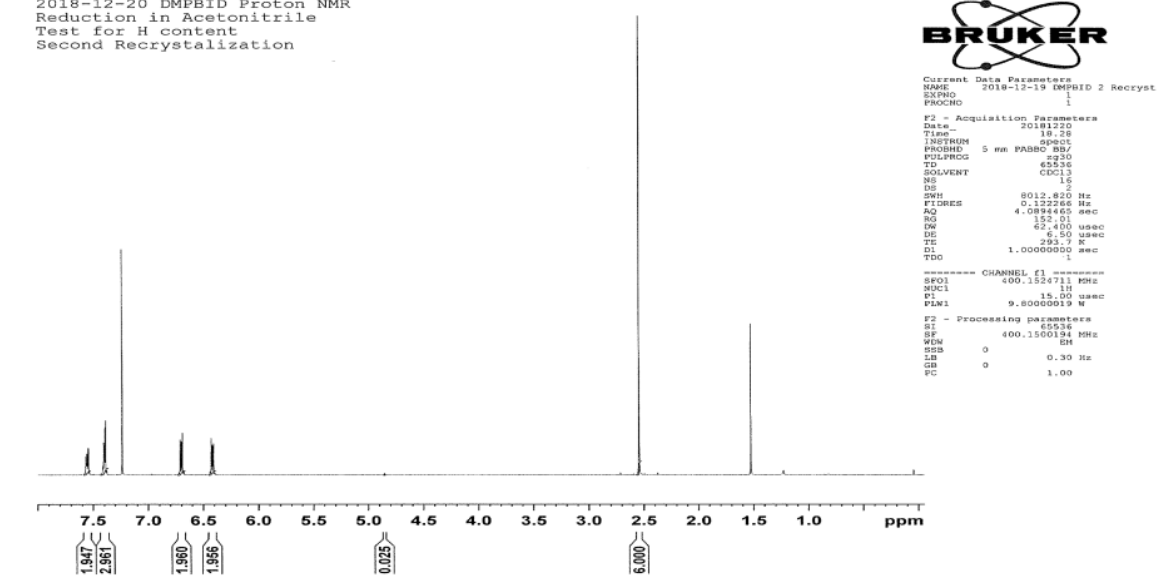

The ${ }^{1} \mathrm{H}$ NMR of DMPBIH-2-d in $\mathrm{CD}_{3} \mathrm{CN}$ 


\section{References}

(1) Maharjan, B.; Raghibi Boroujeni, M.; Lefton, J.; White, O. R.; Razzaghi, M.; Hammann, B. A.; Derakhshani-Molayousefi, M.; Eilers, J. E.; Lu, Y.: Steric Effects on the Primary Isotope Dependence of Secondary Kinetic Isotope Effects in Hydride Transfer Reactions in Solution: Caused by the Isotopically Different Tunneling Ready State Conformations? J. Am. Chem. Soc. 2015, 137, 6653 - 6661.

(2) Powell, M. F.; Bruice, T. C.: Effect of isotope scrambling and tunneling on the kinetic and product isotope effects for reduced nicotinamide adenine dinucleotide model hydride transfer reactions. J. Am. Chem. Soc. 1983, 105, 7139-7149.

(3) Ma, L.; Sakhaee, N.; Jafari, S.; Wilhelm, S.; Rahmani, P.; Lu, Y.: Imbalanced Transition States from $\alpha-H / D$ and Remote $\beta-$ Type N-CH/D Secondary Kinetic Isotope Effects on the NADH/NAD+ Analogues in Their Hydride Tunneling Reactions in Solution. J. Org. Chem. 2019, 84, 5431-5439. 